\title{
A DRUDE MODEL APPROACH TO DISPERSION INTERACTIONS IN DIPOLE-BOUND ANIONS
}

By

Feng Wang

BS, Peking University, Beijing, 1998

Submitted to the Graduate Faculty of

Arts and Sciences in partial fulfillment of the requirements for the degree of Doctor of Philosophy

University of Pittsburgh

April 2003 


\title{
UNIVERSITY OF PITTSBURGH
}

\section{FACULTY OF ARTS AND SCIENCES}

This dissertation was presented

by

\section{Feng Wang}

It was defended on

\author{
April, 15 ${ }^{\text {th }}, 2003$ \\ and approved by \\ Rob D. Coalson \\ Jeffry D. Madura \\ Peter E. Siska
}

Kenneth D. Jordan

Dissertation Director 


\section{Acknowledgements}

I am really grateful to all the people that helped me during the past five years. I learned a lot of my advisor Kenneth. D. Jordan and from all the former group members. Professor Jordan in particular has been very supportive, accessible and helpful in science and in other aspects of my life. I would like to thank Fernando Vila, Arnold Tharrington, Jan Steckel, John Wood, Richard Christie, Brad Tsai, Hanbin Liu, Kadir Diri. All the theory professors in the department of chemistry of university of Pittsburgh have been very supportive. I would like to mention, David Beratan, Rob Coalson and Peter E. Siska. I would also like to thank the recruiting team of University of Pittsburgh. I would not have the opportunity to meet Prof. Jordan and his wonderful research group and get my $\mathrm{Ph}$. D degree here without them.

I would like to thank my parents, who never resented letting their only child travel ten thousand kilometers away to pursuit his dream. I would like to thank my father in-law mother in-law and my wife Zhuoxin for their good personalities, understanding and support.

God bless you all. 


\begin{abstract}
The problem of the binding of an excess electron to polar molecules and their clusters with sufficient attractive electrostatic potential has long fascinated researchers. Although excess electrons bound to such attractive potential tend to be very extended spatially and to have little spatial overlap with the valence electrons of the neutral molecules, inclusion of electron correlation effects is essential for quantitatively describing the electron binding. The major electron correlation contribution may be viewed as a dispersion interaction between the excess electron and the electrons of the molecule or cluster. Intrigued by the success of classical Drude model treatment of neutral atoms or molecules, a new one-electron model based on Drude oscillator was introduced, and its application on $\mathrm{HCN}^{-},(\mathrm{HCN})_{2}{ }^{-}, \mathrm{HNC}^{-},(\mathrm{HNC})_{2}{ }^{-}$and $\left(\mathrm{H}_{2} \mathrm{O}\right)_{\mathrm{n}}{ }^{-}$was studied. The newly developed water potential is used in carrying out parallel-tempering Monte-Carlo simulations of the $\left(\mathrm{H}_{2} \mathrm{O}\right)_{6}{ }^{-}$cluster.
\end{abstract}




\section{Table of Content}

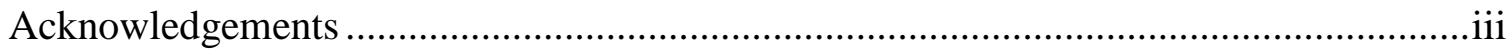

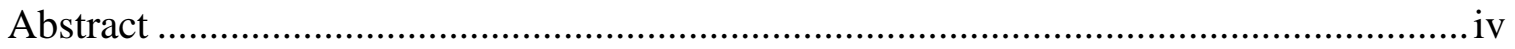

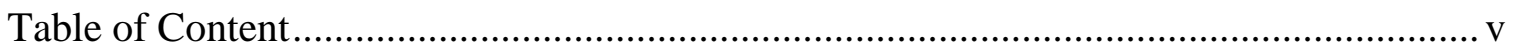

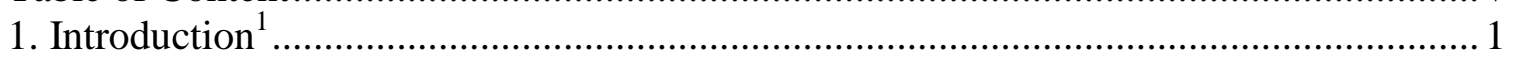

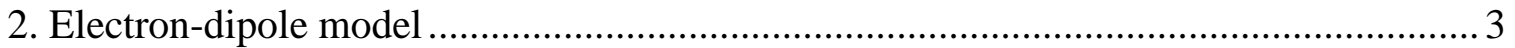

3. Ab initio Treatment of Dipole-bound Anions ………….............................................. 7

4. One-electron model potentials approaches to dipole-bound anions.............................. 10

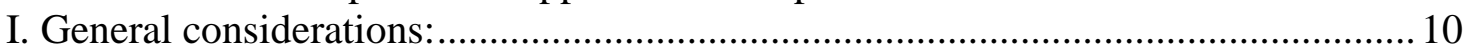

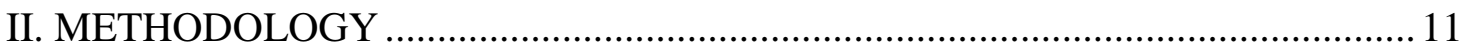

A. Drude model for inter-molecular interactions ................................................... 11

B. Drude model for electron-polar molecule interactions.......................................... 14

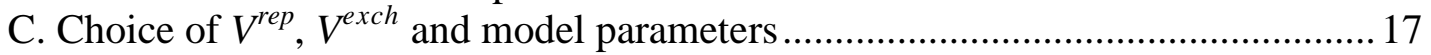

D. Extension of the model potential to clusters ...................................................... 20

E. Ab initio calculations and choice of basis sets ....................................................22

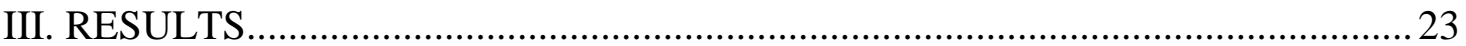

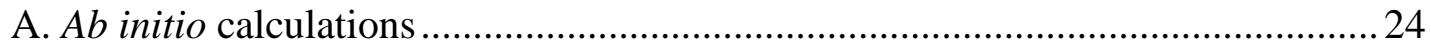

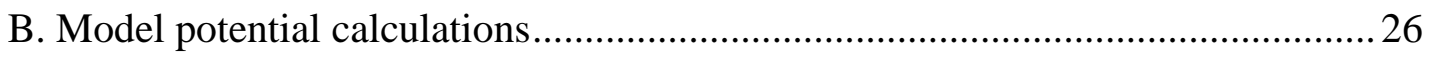

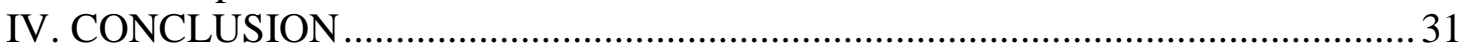

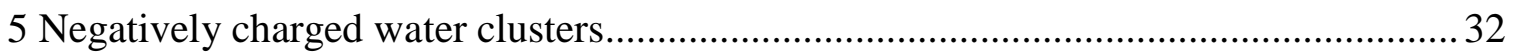

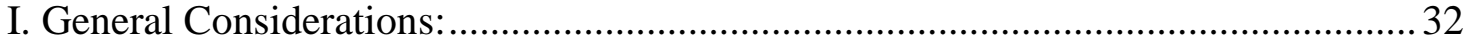

II. Applications of the Drude model to water clusters ................................................ 35

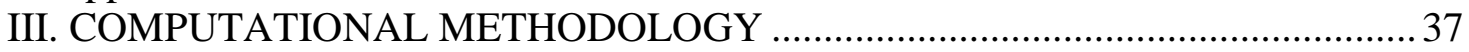

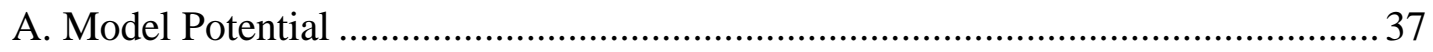

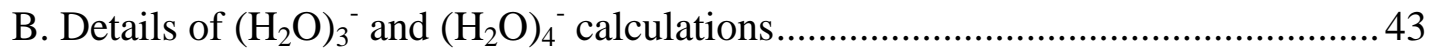

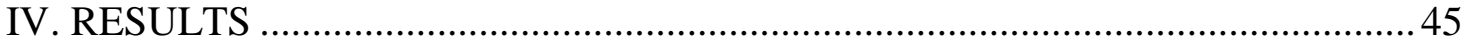

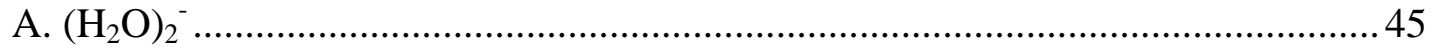

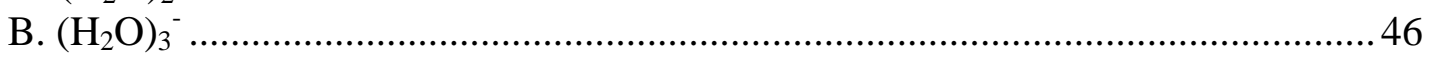

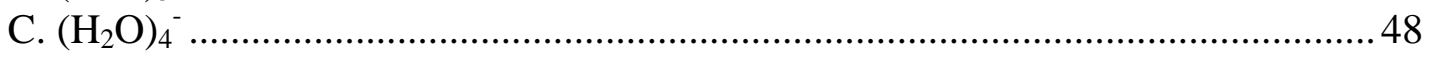

D. Electron densities and importance of single excitations. ...................................... 49

E. Renormalized MP2-level model potential ...................................................... 51

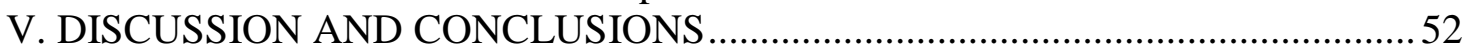

6. Parallel Tempering Monte Carlo Simulations of the Finite Temperature Behavior of

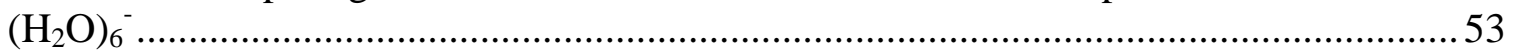

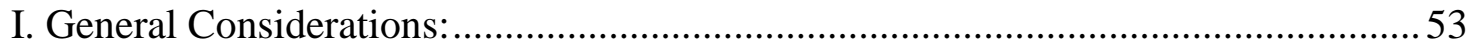

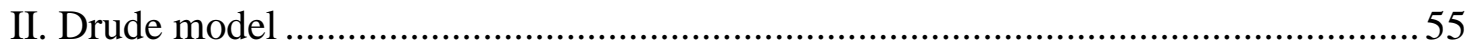

A. Drude Model for electron-molecule interactions …………………….................55

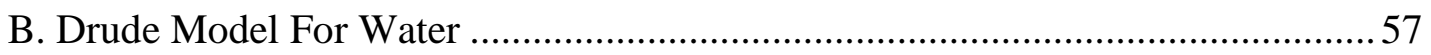

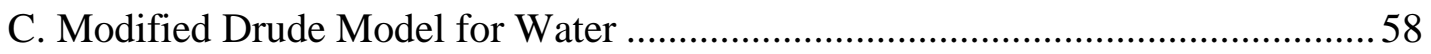

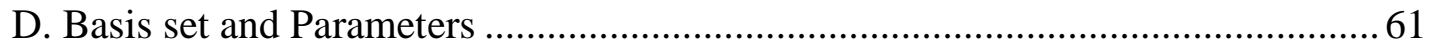

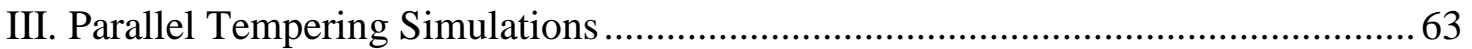

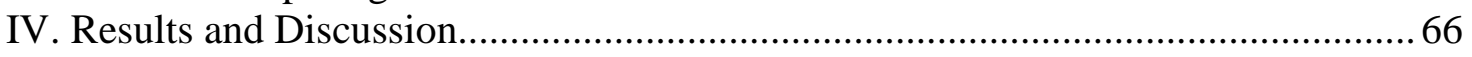

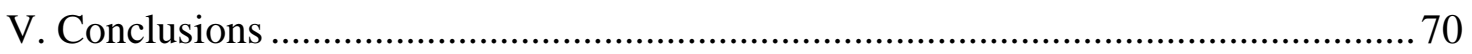

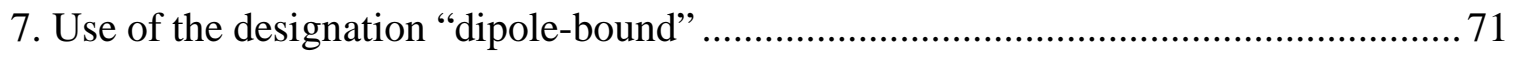




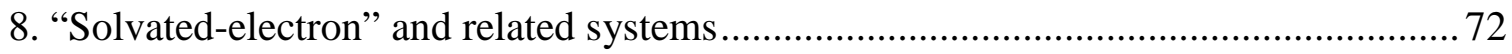

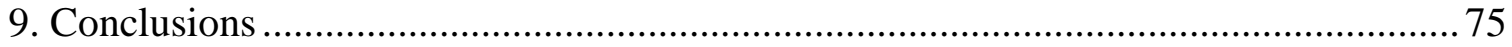

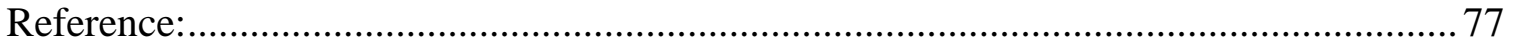

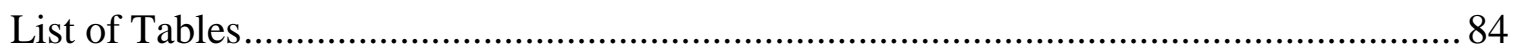

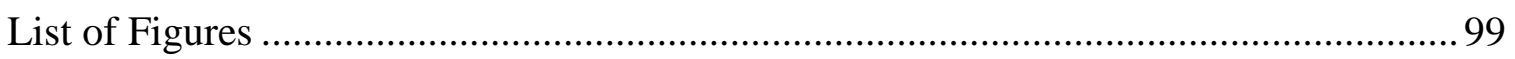

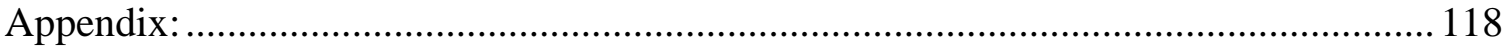

Theoretical Calculations of Voltage-dependent STM Images of Acetylene on the

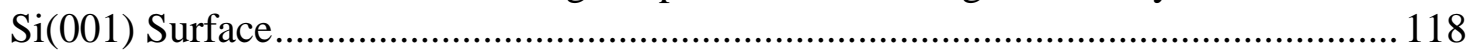

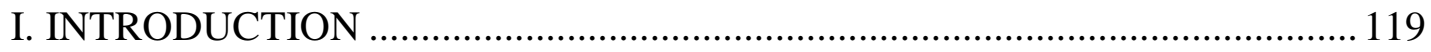

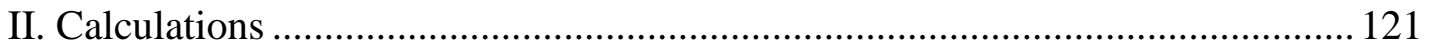

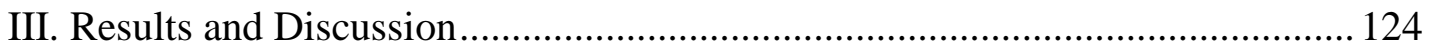

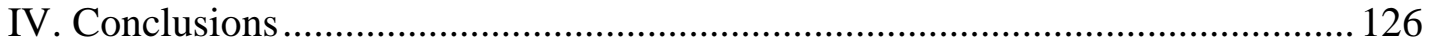

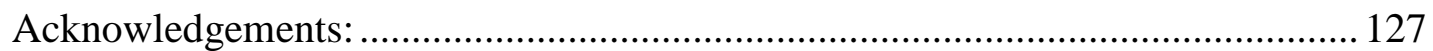

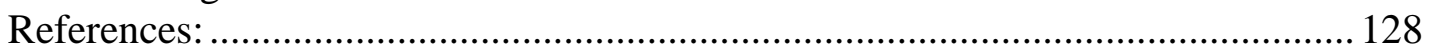




\section{Introduction ${ }^{1}$}

The problem of an electron in the field of a finite fixed dipole comprised of two charges $+Q$ and $-Q$, separated by a distance $R$ has been studied by numerous researchers. ${ }^{2-16}$ The finite dipole system has an infinity of critical dipole moments for electron binding with an infinite number of bound states appearing at each critical moment. The first three critical moments are $1.6248,9.6375$ and $19.181 \mathrm{D}^{3,4}$ It has also been established that the critical moments remain unchanged if a repulsive term falling off more rapidly with distance from the dipole than does the electrostatic interaction is added. ${ }^{4}$ These results imply that, in the absence of corrections to the Born-Oppenheimer (BO) approximation, ${ }^{17}$ "real" molecules with dipole moments greater than 1.625D must possess dipole-bound anion states. The point-dipole problem has the same critical moments as the finite-dipole problem, however, in the absence of a repulsive core, this problem is unphysical in the sense that binding energies become infinite and the wavefunctions are not normalizable. ${ }^{4,15,16}$

For molecules or clusters with dipole moments only slightly in excess of the critical value, the electron binding energies associated with dipole-bound anions are very small and corrections to the BO approximation become important. Indeed, Crawford and Garrett ${ }^{8,18,19}$ have shown that when electronic-rotational coupling is included, the critical dipole moments for binding an excess electron acquire a dependence on the moments of inertia of the molecule, and, as a result, they vary from molecule to molecule. As a "rule of thumb", real molecules have a first critical dipole moment of about 2.4 D. The other important change resulting from inclusion of corrections to the $\mathrm{BO}$ approximation is that the number of bound states is reduced from infinity to a finite number, indeed, only one, 
unless the dipole is very large. ${ }^{20}$ This article will deal primarily with species for which the excess electron is sufficiently strongly bound that corrections to the $\mathrm{BO}$ approximation are negligible.

The above introductory remarks have focused on the critical dipole moments for electron binding. Equally intriguing is the nature of the interaction between the excess electron and the electrons of the polar molecule or cluster of polar molecules. In a Hartree-Fock treatment, the orbital occupied by a dipole-bound electron is very diffuse and polarized away from the molecule on the positive end of the dipole. As a result, it was long believed that, because of the small overlap between the charge distribution of the excess electron and the electrons of the neutral molecule or cluster, electron correlation effects should play a relatively unimportant role in determining the electron binding energies and other properties of dipole-bound anions. ${ }^{21,22}$ However, over the past decade, a rather different picture has emerged, and, it is now known that electron correlation effects can drastically alter the properties of dipole-bound anions. ${ }^{23-30}$ The main correlation contribution is a dispersion-type interaction between the excess electron and the electrons of the polar molecule of the cluster. ${ }^{23-27}$ In many cases fourth- and higher-order correlation effects also make sizable contributions to the electron binding energies. Thus, an accurate description of the dipole-bound anions of these systems using conventional ab initio electron structure methods requires treating electron correlation effects to high order, e.g., by means of the $\operatorname{CCSD}(\mathrm{T})$ or CCSDT coupled-cluster methods, ${ }^{31,32}$ and using large, flexible basis sets. In light of this, it is of considerable interest that it is possible to develop one-electron models that recover most of the correlation contributions to the electron binding to such species. ${ }^{33-37}$ This finding, in turn, 
may suggest new ways of tackling the dispersion problem in general.

Dipole-bound anions were long considered to be rather esoteric, albeit fascinating, entities, without much relevance to mainstream chemistry. However this is no longer the case, as they have been found to be important in a variety of chemical processes. The demonstration that dipole-bound anions can serve as precursors to formation of valencelike anions ${ }^{38-40}$ has generated much interest in their role in electron capture in biological molecules such as uracil and thymine. ${ }^{41-46}$ In addition, many biologically important molecules can exist as zwitterions, which because of their large dipole moments, can form dipole-bound anions. ${ }^{46-50}$ Dipole-bound anions have also been found to be important in charge-transfer processes. For example, photo-excitation of $\mathrm{I}^{-} \cdot\left(\mathrm{H}_{2} \mathrm{O}\right)_{4}$ leads to the charge transfer-complex $\mathrm{I} \cdot\left(\mathrm{H}_{2} \mathrm{O}\right)_{4}{ }^{-}$, with the excess electron bound to the cyclic $\left(\mathrm{H}_{2} \mathrm{O}\right)_{4}$ cluster, distorted so that it has a large dipole moment. ${ }^{51-56}$. Dipole-bound anions have also been invoked in explaining diffuse interstellar absorption bands. ${ }^{57}$

The recent resurgence of interest in dipole-bound anions and the closely related "solvated-electron" systems has been fueled by important advances on the experimental front. These advances include the use of Ar-atom vibrational predissociation spectroscopy, enabling one to obtain vibrational spectra of cold anionic clusters, ${ }^{40,58,59}$ femtosecond photodetachment spectroscopy, ${ }^{60}$ a new generation of experiments using electron transfer from highly excited Rydberg states, ${ }^{61,62}$ and improved field ionization methods ${ }^{63}$ for estimating the electron binding energies of weakly bound anions.

\section{Electron-dipole model}

Although the primary focus of the article is on electron correlation effects in electron-polar molecule interactions, it is instructive to first consider the energy levels of 
an electron bound to a finite fixed dipole, where two fixed point charges $+Q$ and $-Q$ are separated by a distance $\overrightarrow{\mathbf{R}}$, with the quantity $\overrightarrow{\boldsymbol{\mu}}=Q \overrightarrow{\mathbf{R}}$ giving the dipole moment. The Hamiltonian is the same as that for $\mathrm{H}_{2}{ }^{+}$, except for the change of the sign of one of the "nuclei". ${ }^{64}$

The Schrödinger equation in atomic units for an electron in such a system is:

$$
\left[-\frac{1}{2} \nabla^{2}-\frac{Q}{r_{+}}+\frac{Q}{r_{-}}\right] \boldsymbol{\Psi}=E \boldsymbol{\Psi},
$$

where $r_{+}$and $r_{-}$are the distances from the electron to the positive and negative charges, respectively. This equation is decoupled into three ordinary differential equations in elliptic coordinates $\lambda, \eta$ and $\phi$, where:

$$
\lambda=\frac{r_{+}+r_{-}}{R}, \quad \eta=\frac{r_{+}-r_{-}}{R}
$$

and $\phi$ is the azimuth angle of rotation around the dipole axis. Upon variable substitution Eq. 1 becomes

$$
\begin{gathered}
\frac{d}{d \lambda}\left[\left(\lambda^{2}-1\right) \frac{d \mathrm{~L}}{d \lambda}\right]+\left[p^{2} \lambda^{2}+A-\frac{m^{2}}{\lambda^{2}-1}\right] \mathrm{L}=0, \\
\frac{d}{d \eta}\left[\left(1-\eta^{2}\right) \frac{d \mathrm{~N}}{d \eta}\right]+\left[p^{2} \eta^{2}-2 \mu \eta-A-\frac{m^{2}}{1-\eta^{2}}\right] \mathrm{N}=0, \\
\frac{d^{2} \Phi}{d \phi^{2}}+m^{2} \Phi=0,
\end{gathered}
$$

and

$$
\boldsymbol{\Psi}=\mathrm{L}(\lambda) \mathrm{N}(\eta) \Phi(\phi)
$$

$A$ is a separation constant and 


$$
p^{2}=\frac{\mu^{2} E}{2} .
$$

It is easily shown that $m$ is the modulus of the magnetic quantum number since the obvious solution to Equation $3 \mathrm{c}$ is

$$
\Phi(\phi)=\exp ( \pm i m \phi)
$$

The problem is has a closed solution in the united atom limit $(R \rightarrow 0)$ and at infinite separation $(R \rightarrow \infty)$, however, there is no general solution to Equation 3a for intermediate distances. Fortunately, when the electron binding energy $E \rightarrow 0$, thus $p \rightarrow 0$, the above equation reduces to an equation of $\mu$ only and is analytically solvable using an infinite series of Legendre functions. The critical dipole moment for electron binding thus does not depend on $Q$ and $R$ separately. The bound states can be labeled using three quantum numbers $\left(n_{\lambda}, n_{\eta}\right.$ and $\left.m\right)$, with the constraint,

$$
n=n_{\lambda}+n_{\eta}+m+1
$$

Figure 1 reports as a function of the dipole moment $(\mu)$ the energies of the lowlying levels of this model in the case that the two point charges are +1 and -1 . In the large dipole limit, the spectrum corresponds to that of the $\mathrm{H}$ atom Stark-shifted by a -1 point charge.

All of the energy levels associated with the first critical moment $\left(\mu_{1}=1.625 \mathrm{D}\right)$ are $\sigma$-like, with the most stable level correlating with the $n=1$ hydrogenic level in the $R$ $\rightarrow \infty$ limit. The higher-lying levels associated with this critical moment correlate with excited states of the $\mathrm{H}$ atom, with one level correlating with each of the $n \geq 2 \mathrm{H}$-atom levels. All levels associated with the second critical moment of $9.637 \mathrm{D}$ are $\pi$-like, with the lowest of these correlating with the $2 p_{\pi}$ orbital of the $\mathrm{H}$ atom. Finally, the lowest 
energy level associated with the third critical moment of 19.101 D, correlates with the unfavorably hybridized $2 s-2 p_{\sigma}$ hydrogenic orbital. There is an infinity of larger critical dipole moments, but these are unimportant for most purposes. The reader may note that levels of the same apparent symmetry are shown as crossing in Figure 1. This is not a violation of the non-crossing rule, as the electron finite-dipole problem, like $\mathrm{H}_{2}{ }^{+}$, admits an additional constant of the motion. ${ }^{64,65}$

Figure 2 displays the charge distributions of the ground electronic state of the electron-finite-dipole system for four different choices of the parameters: $Q=1$ and $\mu=3$ or $6 \mathrm{D}$ (Figure $2 a$ ), and $Q=0.5$ and $\mu=3$ or $6 \mathrm{D}$ (Figure $2 b$ ). From Figures $2 a$ and $2 b$, it is seen that the polarization of the wavefunction away from the negative end of the dipole is, as expected, much greater for smaller dipole moments and that the electron density is more localized for the higher charges (keeping the dipole moment fixed).

The most serious shortcoming of the finite dipole model is that it gives electron binding energies much larger than those determined experimentally for real molecules with the same dipole moments. This is primarily a consequence of the neglect of repulsive interactions of the excess electron with the electrons of the neutral molecule. In general, there is appreciable electron density on the atom or atoms constituting the positive end of the dipole, and, as a result, there is an "excluded-volume effect", which reduces electron binding.

This "excluded-volume effect" can be readily incorporated into one-electron models by adding a repulsive term to the Hamiltonian describing the interaction of the excess electron with the distribution of charges of the neutral molecule. ${ }^{66}$ Figures $2 c$ and $2 d$ report the electron charge distributions and binding energies for the four models 
considered above modified to include a repulsive term centered on the positive end of the dipole. The repulsive core was represented by a single Gaussian function with an exponent of 10 and a prefactor of 1.0, and is roughly comparable to that appropriate for describing binding of an excess electron to $\mathrm{HCN} .{ }^{33}$ As expected, introduction of the repulsive core leads to a decreased electron binding and a more extended charge distribution of the excess electron.

The point-dipole plus repulsive core model is a static model in that it does not allow for relaxation of the "core" electrons upon the attachment of the excess electron, nor does it allow for correlation interactions between the dipole-bound electron and the core electrons. To include such effects it would appear to be necessary to adopt $a b$ initio methods, treating explicitly all the electrons or at least the valence electrons together with the dipole-bound electron. However, as mentioned in the Introduction, it is, in fact, possible to describe relaxation and the dominant correlation effects within a one-electron model. Before describing one-electron model approaches, we first consider ab initio treatments of dipole-bound anions.

\section{Ab initio Treatment of Dipole-bound Anions}

In discussing ab initio approaches to characterizing dipole-bound anions, it is useful to start with the Koopmans' Theorem (KT) approximation ${ }^{67}$ in which the electron affinity (the negative of electron binding energy) is given by

$$
E A^{K T}=-\varepsilon_{L U M O}
$$

where $\varepsilon_{L U M O}$ is the energy of the lowest unoccupied molecular orbital obtained from a Hartree-Fock calculation on the neutral molecule. This is a static approximation in that 
the electron binds in the field of the static potential of the ground state of the neutral molecule or clusters and neither electron relaxation nor correlation effects are included. The "excluded volume" effect discussed above is automatically included in ab initio treatments. For molecules or clusters with dipole moments greater than $1.625 \mathrm{D}, \varepsilon_{L U M O}$ is necessarily negative, i.e., the LUMO is bound, provided the basis set is sufficiently flexible and the Born-Oppenheimer approximation is made.

The relaxation contribution to the electron binding can be obtained by carrying out Hartree-Fock calculations on the anion and neutral species, and using the expression

$$
\Delta E^{\text {relax }}=-E A^{K T}-\left(E_{\text {anion }}^{H F}-E_{\text {neutral }}^{H F}\right) .
$$

Electron correlation contributions to the binding energy can be calculated by a variety of methods, including many-body perturbation theory or coupled-cluster theory. Regardless of the theoretical method employed, it is useful to decompose the EA as follows:

$$
E A=E A^{K T}+\Delta E^{\text {relax }}+\Delta E^{(2)-d i s p}+\Delta E^{(2)-n o n d i s p}+\Delta E^{(3)}+\Delta E^{(4)}+\Delta E^{H O} .
$$

In this expression, the second-order correlation correction, $\Delta E^{(2)}$, has been separated into dispersion and non-dispersion components. ${ }^{23} \Delta E^{(3)}$ and $\Delta E^{(4)}$ give, respectively, the thirdand fourth-order corrections to the electron binding energy, and $\Delta E^{H O}$ collects together fifth- and higher-order corrections. $\Delta E^{H O}$ can be estimated, for example, by comparing the EA from MP4(SDTQ) and CCSD(T) calculations.

High-level electronic structure calculations on a variety of dipole-bound anions have revealed that electron correlation effects play a major role in describing the binding of the excess electron in these systems. ${ }^{23-26,28,30,33,34,68,69}$ This may be seen from examination of Table I, which summarizes the results of ab initio calculations of the vertical electron detachment energies, decomposed along the lines Eq. (9), of $\mathrm{HCN}^{-}$, 
$(\mathrm{HCN})_{2}^{-},\left(\mathrm{H}_{2} \mathrm{O}\right)_{2}^{-},(\mathrm{HF})_{2}^{-},(\mathrm{HF})_{3}^{-}$, and $\mathrm{CH}_{3} \mathrm{CN}^{-}$. Where available, experimental vertical electron detachment energies are included for comparison. Table I also reports, the dipole moments of the neutral molecules (or clusters) calculated at the Hartree-Fock and correlated (MP2 or QCISD ${ }^{31}$ ) levels of theory. The calculations have been carried out using flexible Gaussian-type orbital basis sets that are expected to give electron binding energies and dipole moments very close to the complete-basis-set limit values. The second-order contributions to the electron binding energies have been decomposed into dispersion and non-dispersion contributions using a procedure of Gutowski et al. ${ }^{23,26,70}$

Analysis of the results in Table I reveals that, for the molecules and clusters considered, the relaxation contributions to the electron binding energies vary from 4 to $10 \%$ of $\varepsilon^{\mathrm{KT}}$, whereas, the second-order dispersion contributions are much more important, ranging from 47 to $107 \%$ of $\varepsilon^{\mathrm{KT}}$. The non-dispersion contributions act so as to decrease the electron binding. This is attributed to the fact that for the species considered, electron correlation effects reduce the dipole moment of the neutral molecules. ${ }^{70}$ As a result, the second-order dispersion and non-dispersion contributions to the electron binding energies are of opposite sign, and thus partially cancel. The third-order corrections to the electron binding energies tend to be relatively small, but the fourth- and higher-order corrections are sizable in many cases. Indeed, for $\left(\mathrm{H}_{2} \mathrm{O}\right)_{2}{ }^{-}, \Delta \mathrm{E}^{\mathrm{HO}}$ is comparable to the $\mathrm{KT}$ binding energy. The origin of the large fourth- and higher-order corrections to the electron binding energies of many dipole-bound anions has proven elusive. We return to this issue later in the review.

In general, good agreement is found between the electron binding energies from $\operatorname{CCSD}(\mathrm{T})$ calculations and those measured experimentally, and, in those cases where 
there are sizable discrepancies $\left(\right.$ e.g., $(\mathrm{HF})_{2}{ }^{-}$and $\left.\mathrm{CH}_{3} \mathrm{CN}^{-}\right)$, it is expected that these would be resolved by carrying out CCSDT or multi-reference coupled-cluster calculations. (The $\operatorname{CCSD}(\mathrm{T})$ procedure treats triple excitations in a perturbative manner, whereas the CCSDT method treats these excitations non-perturbatively.) The important of nonperturbative triples for the dipole bound anions of $\mathrm{HCN}$ and $\mathrm{HNC}$ has been discussed by Peterson and Gutowski. ${ }^{32}$ The realization that $\mathrm{CCSD}(\mathrm{T})$ or CCSDT methods together with large flexible basis sets are required to describe accurately dipole bound anions is disconcerting as such calculations are computationally prohibitive for the large molecule or cluster systems currently being studied experimentally and theoretically ${ }^{42,45,71-73}$ For these systems, it has often been necessary to compromise on the theoretical method employed.

\section{One-electron model potentials approaches to dipole- bound anions}

\section{General considerations:}

Given the diffuse, extended charge distribution of excess electrons bound to such clusters, these appear to be ideal systems to describe using model potential approaches. Indeed, there is a rich literature describing applications of model potentials for treating the interactions of an excess electron with water clusters and with bulk water. ${ }^{63,74-81}$ In general, these approaches employ a standard classical force field for describing the interactions between the neutral monomers and a model potential for describing the interaction of the excess electron with the monomers. The excess electron is treated quantum mechanically. 
All existing model potentials for electron-polar molecule systems allow for the electrostatic and, generally also, short-range repulsive interactions between the excess electron and the monomers. In some cases, polarization of the monomers and the exchange interaction between the excess electron and the monomers have also been included. However, none of the model potential approaches introduced to date has allowed explicitly for "dispersion" interactions between the excess electron and the molecules in the cluster. This is a potentially serious limitation, since, as noted above, such interactions can significantly enhance electron binding. In this article we present a Drude-type model ${ }^{82}$ for describing the dispersion interaction between an excess electron and a polar molecule or cluster of polar molecules. The method is demonstrated in applications to $\mathrm{HCN},(\mathrm{HCN})_{2}, \mathrm{HNC}$ and $(\mathrm{HNC})_{2}$. The theoretical approach is described in the next section, followed by a presentation of the results for the test systems.

\section{METHODOLOGY}

\section{A. Drude model for inter-molecular interactions}

The Drude oscillator approach was originally introduced to describe dispersion interactions between atoms or molecules. ${ }^{82}$ In order to describe the dispersion interaction for a system of two molecules, a Drude oscillator is associated with each molecule. A Drude oscillator is a pair of charges $+q$ and $-\mathrm{q}$ coupled through a harmonic oscillator. When observed from a distance longer than the dimension of the Drude oscillator, the Drude oscillator looks like a harmonic oscillating dipole, which is used to represent the spontaneous oscillating dipole of the molecule, the interaction of which is responsible for 
the dispersion interaction. The interaction of two dipoles separated by a distance $r$ significantly longer than the magnitude of the dipole can be represented as

$$
U_{A B}=\frac{\mu_{A} \mu_{B}}{r^{3}}\left(\sin \theta_{A} \sin \theta_{B} \cos \phi-2 \cos \theta_{A} \cos \theta_{B}\right)
$$

where the angles $\theta_{\mathrm{A}}, \theta_{\mathrm{B}}$, and $\phi$ is defined in Figure 3 .

The Hamiltonian of two interacting Drude oscillators, positioned along the $\mathrm{z}$ axis, has the form

$$
\begin{gathered}
-\frac{1}{2} \nabla_{1}^{2}-\frac{1}{2} \nabla_{2}^{2}+\frac{1}{2} k\left(x_{1}^{2}+y_{1}^{2}+z_{1}^{2}\right)+\frac{1}{2} k\left(x_{2}^{2}+y_{2}^{2}+z_{2}^{2}\right) \\
+\frac{x_{1} x_{2} q^{2}}{r^{3}}+\frac{y_{1} y_{2} q^{2}}{r^{3}}-\frac{2 z_{1} z_{2} q^{2}}{r^{3}}
\end{gathered} .
$$

Atomic units are used in this and all subsequent equations unless specified otherwise. The system of interacting oscillators can be viewed as non-interacting oscillators by making the following substitutions:

$$
\begin{aligned}
& X_{1}=\frac{x_{1}+x_{2}}{\sqrt{2}} ; X_{1}=\frac{x_{1}-x_{2}}{\sqrt{2}} ; K_{1 x}=k+\frac{q^{2}}{r^{3}} ; K_{2 x}=k-\frac{q^{2}}{r^{3}} \\
& Y_{1}=\frac{y_{1}+y_{2}}{\sqrt{2}} ; Y_{1}=\frac{y_{1}-y_{2}}{\sqrt{2}} ; K_{1 x}=k+\frac{q^{2}}{r^{3}} ; K_{2 y}=k-\frac{q^{2}}{r^{3}} \\
& Z_{1}=\frac{z_{1}+z_{2}}{\sqrt{2}} ; Z_{1}=\frac{z_{1}-z_{2}}{\sqrt{2}} ; K_{1 z}=k-\frac{2 q^{2}}{r^{3}} ; K_{2 z}=k+\frac{2 q^{2}}{r^{3}}
\end{aligned}
$$

The corresponding angular frequency of the non-interacting oscillators can be shown to relate to the angular frequency of the isolated Drude oscillator as follows:

$$
\begin{aligned}
& \omega_{1 x}=\omega \sqrt{1-\frac{q^{2}}{r^{3} k}} ; \omega_{2 x}=\omega \sqrt{1+\frac{q^{2}}{r^{3} k}} \\
& \omega_{1 y}=\omega \sqrt{1-\frac{q^{2}}{r^{3} k}} ; \omega_{2 y}=\omega \sqrt{1+\frac{q^{2}}{r^{3} k}}
\end{aligned}
$$




$$
\omega_{1 z}=\omega \sqrt{1-\frac{2 q^{2}}{r^{3} k}} ; \omega_{2 z}=\omega \sqrt{1+\frac{2 q^{2}}{r^{3} k}}
$$

The ground state energy of the interacting oscillators is given by the sum of the zeropoint energies of all six normal modes in Equation (13). The expansion of the ground state energy using Taylor series and accurate to the third order $\mathrm{q}^{2} /\left(\mathrm{kr}^{3}\right)$ gives:

$$
E_{0}(r)=3 \omega-\frac{3 q^{4} \omega}{4 k^{2} r^{6}}-o\left(\frac{q^{6}}{k^{3} r^{9}}\right)
$$

The second term in Equation (14) is dispersion energy and higher order corrections to the ground state energy are all negative.

If an electrostatic field $\boldsymbol{E}$ is applied to a Drude oscillator along the $\mathrm{z}$ direction, the charge separation of the Drude oscillator takes the form

$$
z=\frac{q E}{k}
$$

The perturbed oscillator has a dipole moment

$$
\mu=q z=\frac{q^{2}}{k} E
$$

The polarizability of Drude oscillator thus has the form

$$
\alpha=\frac{q^{2}}{k}
$$

Thus, the dispersion energy in Equation 14 becomes

$$
E_{0}(r)=-\frac{3 \alpha^{2} \omega}{4 r^{6}}
$$

The negative sign indicates that the dispersion energy stabilizes the system. 


\section{B. Drude model for electron-polar molecule interactions}

The Drude model can be extended to treat the interaction between an excess electron and an arbitrary polarizable molecule and cluster of polarizably molecules. We first restrict our discussion to a Drude model approach for the interaction between an excess electron and a molecule or cluster of molecules that have sufficient high dipole or high-order moments that an electron binds even without dispersion-type interactions. If the system of interest does not bind an electron without dispersion-type interactions, the Drude model can still be employed. In this case, the electron-Drude oscillator interaction has to be included into the zeroth order Hamiltonian instead of being treated as a small perturbation.

We first consider the case of the interaction of an excess electron with a single polar molecule. The extension to the case of an electron interacting with a molecular dimer is given in Subsection. D.

For an electron interacting with a polar molecule, the Hamiltonian may be written as

$$
H=H^{e l}+H^{\text {osc }}+V^{\text {couple }},
$$

where $H^{e l}$ and $H^{o s c}$ are the Hamiltonians for the excess electron/polar molecule system

and the Drude oscillator, respectively, and $V^{\text {couple }}$ is the coupling term, which incorporates the effects of both polarization and dispersion.

$H^{e l}$ is defined as:

$$
H^{e l}=-\frac{1}{2} \nabla_{e}^{2}-\sum_{i} \frac{Q_{i}}{r_{i}}+V^{r e p}+V^{e x c h},
$$


where it is assumed for simplicity that charge distribution of the neutral molecule is well represented as a set of point charges $\left\{Q_{i}\right\}$ located at positions $\mathbf{r}_{\mathrm{i}} . V^{\text {rep }}$ and $V^{\text {exch }}$ represent, respectively, the short-range repulsion and exchange interactions between the excess electron and the molecule core. (The various interaction terms are given in atomic units.)

The Drude oscillator consists of two "fictitious" charges $+q$ and $-q$ separated by distance $R$ and coupled harmonically through the force constant $k$. The $+q$ charge is taken to be fixed, whereas the $-q$ charge can be displaced. (See Fig. 4 for definitions of the coordinates.) The mass associated with the oscillator is taken to be $m_{o}$. In the absence of an interaction with an external field (e.g., that imposed by the excess electron), the average of $\mathbf{R}$ is zero. The Hamiltonian for the three-dimensional Drude oscillator is given by

$$
H^{o s c}=-\frac{1}{2 m_{o}} \nabla_{o}^{2}+\frac{1}{2} k\left(X^{2}+Y^{2}+Z^{2}\right),
$$

where $\left(X^{2}+Y^{2}+Z^{2}\right)=R^{2}$.

The coupling term is taken to be

$$
V^{\text {couple }}=\frac{q \mathbf{r} \cdot \mathbf{R}}{r^{3}}
$$

where $\mathbf{r}$ is the vector position of the excess electron relative to the $+q$ charge and $\mathbf{R}$ gives the position of the $-q$ charge . This treatment ignores the higher order (i.e., nondipolar) moments induced on the molecule by the excess electron.

The energy levels of the Hamiltonian given by Eq. (19) are calculated using a product basis set of the form:

$$
\psi=\phi_{\beta} \chi_{i}
$$

where $\phi_{\beta}$ is an orbital associated with $H^{e l}$ and $\chi_{i}$ is an eigenfunction $H^{o s c}$. 
In our application of the one-electron model potential approach to electron binding to polar molecules and their clusters, we will report electron binding energies calculated at zeroth- and second-order as well as with the configuration interaction method, which includes interactions to all orders. The zeroth-order ground state energy $E^{(0)}$ is simply the sum of the ground state energies associated with the uncoupled electronic and oscillator Hamiltonians. There is no first-order correction to the energy. The second-order energy correction can be decomposed into two contributions:

$$
E^{(2)}=\sum_{i \neq 0} \frac{\left\langle 00\left|V^{\text {couple }}\right| 0 i\right\rangle^{2}}{E_{00}-E_{0 i}}+\sum_{i \neq 0, \beta \neq 0} \frac{\left\langle 00\left|V^{\text {couple }}\right| \beta i\right\rangle^{2}}{E_{00}-E_{\beta i}}
$$

where $\mid 00>$ denotes the zeroth-order wavefunction and $E_{\beta i}$ represents the energy of the non-interacting electron-oscillator system with the electron in orbital $\phi_{\beta}$ and the oscillator in level $\chi_{i}$. The first term on the right hand side of the above equation gives the polarization energy of the oscillator due to its interaction with the excess electron. The second term, which involves excitations of both the excess electron and the oscillator, gives the dispersion energy. Given the form of $H^{\text {couple }}$, only the lowest excited states of the harmonic oscillator (i.e., those involving one quanta of excitation) contribute to the second-order energy expression.

The polarization contribution in Eq. (24) is proportional to $q^{2} / k$, which corresponds to the polarizability $(\alpha)$ of the oscillator. The mass associated with the Drude model does not enter into this term. However, it does enter the second term on the righthand side of Eq. (24), which involves contributions of the form 


$$
\frac{\frac{q^{2}}{2 k}\left|\left\langle 0\left|\frac{s}{r^{3}}\right| \beta\right\rangle\right|^{2}}{-1+\left(\varepsilon_{0}-\varepsilon_{\beta}\right) \sqrt{\frac{m_{o}}{k}}},
$$

where $\varepsilon_{0}$ and $\varepsilon_{\beta}$ correspond to ground and excited states of $H^{e l}$, and $s=x, y$, or $z$.

$\left(\varepsilon_{0}-\varepsilon_{\beta}\right) \sqrt{\frac{m_{o}}{k}}$ can also be written as $\frac{\left(\varepsilon_{0}-\varepsilon_{\beta}\right)}{\omega_{o}}$ where $\omega_{o}$ is the frequency of the Drude oscillator. Because the dipole-bound electron usually is weakly bound, the most important such terms are expected to have $\left|\left(\varepsilon_{0}-\varepsilon_{\beta}\right) \sqrt{\frac{m_{o}}{k}}\right|<<1$. Thus, to a good approximation, the dispersion contribution to the energy is proportional to $\alpha \sum_{\beta \neq 0}\left|\left\langle 0\left|\frac{s}{r^{3}}\right| \beta\right\rangle\right|^{2}$, and the sensitivity to the choice of the mass of the oscillator should be quite weak. If the excess electron were highly localized at a fixed distance from the Drude oscillator, the term for dispersion interaction would reduce to a London-type expression.

\section{Choice of $V^{\text {rep }}, V^{e x c h}$ and model parameters}

In using Eq. (20) to study electron binding to polar molecules, it is necessary to choose functional forms for $V^{\text {rep }}$ and $V^{\text {exch }}$ and to adopt appropriate charges $\left\{Q_{i}\right\}$. The extension to clusters of polar molecules will be discussed in section II.C.

Following Schnitker and Rossky, ${ }^{75}$ the repulsive potential $V^{\text {rep }}$ is determined by enforcing orthogonality between an orbital occupied by the excess electron and the filled orbitals of the neutral molecule. The starting point for the derivation of the repulsive potential is the following equation, 


$$
\Phi=\phi-\sum_{m} b_{m} \gamma_{m}
$$

where $\Phi$ is an orbital associated with the excess electron in an all-electron Hartree-Fock treatment, and $\phi$ is a pseudo-orbital which is identical to the true orbital outside the core, but which lacks the rapid oscillations inside the core region. The $\left\{\gamma_{m}\right\}$ are the filled molecular orbitals. When combined with $F \Phi=\varepsilon \Phi$, this leads to the equation:

$$
\left\{F-\sum_{m}\left(\varepsilon_{m}-\varepsilon\right) \gamma_{m}\left\langle\gamma_{m} \mid \phi\right\rangle / \phi\right\} \phi=\varepsilon \phi
$$

where $F$ is the Fock operator, $\varepsilon$ is the energy associated with orbital $\Phi$, and $\varepsilon_{m}$ is the energy of the filled orbital $\gamma_{m}$. The second term in the bracket of Eq. (27) defines $V^{\text {rep }}$ :

$$
V^{r e p}=-\sum_{m}\left(\varepsilon_{m}-\varepsilon\right) \gamma_{m}\left\langle\gamma_{m} \mid \phi\right\rangle / \phi
$$

To simplify the solution of Eq. (27), two approximations introduced by Schnitker and Rossky are adopted. Firstly, $\varepsilon$ is neglected in the sum, which is justified since the excess electron interacts weakly with the neutral host (i.e., $\left.|\varepsilon| \ll\left|\varepsilon_{m}\right|\right)$; and secondly, the variation of $\phi$ inside the core region is neglected. As is discussed below, the second approximation causes $V^{r e p}$ to be too repulsive.

With these approximations the second term in the bracket of Eq. (27) reduces to

$$
V^{r e p}(\vec{r})=\sum_{j} C_{j} \xi_{j}(\mathbf{r})
$$

where

$$
C_{j}=-\sum_{m} \varepsilon_{m} K_{m} c_{j}^{m}
$$

with

$$
K_{m}=\sum_{l} c_{l}^{m} \int \xi_{l}(\mathbf{r}) d \mathbf{r}
$$


where $c_{j}^{m}$ is the coefficient of the $j^{\text {th }}$ basis function in the $m^{\text {th }}$ occupied MO. In SchnitkerRossky paper, the $\left\{\xi_{j}(\vec{r})\right\}$ are Slater-type functions. Here generalized Gaussians of the form

$$
\xi_{j}(\vec{r})=x^{l} y^{m} z^{n} e^{-\alpha_{i} r^{2}}
$$

are used instead. Since Gaussian-type basis functions will also be used for representing the excess electron orbitals, this choice facilitates the evaluation of the integrals over $V^{\text {rep }}$.

In the present work the repulsive potentials were determined from Hartree-Fock calculations using the aug-cc-pVTZ basis set ${ }^{83}$ and QCISD/aug-cc-pVTZ and MP2/augcc-pVDZ optimized geometries for $\mathrm{HNC}^{84}$ and $\mathrm{HCN}^{27}$, respectively. The resulting potentials when employed in Eq. (20) result in significant underbinding of the excess electron. This is a consequence of their being too repulsive due to the neglect of the variation of $\phi$ within the core. ${ }^{85}$ To deal with this problem, the repulsive potentials were "weakened" by multiplying by a constant factor so that the electron binding energies calculated for the monomers reproduce the KT results from all-electron Hartree-Fock calculations.

In their work on electron-water cluster systems, Schnitker and Rossky found the exchange contributions to the electron binding energies to be very small, and as a result, they omitted the $V^{\text {exch }}$ term from their model Hamiltonian. ${ }^{75}$ This is a consequence of the small overlap between the orbital occupied by the excess electron and the charge distribution of the neutral molecules. We also adopt this approximation in the present treatment of electron-HCN and electron-HNC interactions.

In applying the model Hamiltonian approach to $\mathrm{HCN}$ and $\mathrm{HNC}$, three atomcentered point charges were employed to represent the monomer charge distributions. 
The point charges were obtained by fitting the electrostatic potentials of $\mathrm{HCN}$ and $\mathrm{HNC}$ calculated at both Hartree-Fock and CCSD(T) levels of theory, using the aug-cc-pVTZ basis set, and employing the same geometries as used in determining the repulsive potentials. ${ }^{86}$ The fitted charges were constrained to reproduce the calculated (either HF or $\operatorname{CCSD}(\mathrm{T})$ level) dipole moments, and were also found to closely reproduce the molecular quadruple moments.

We next consider the choice of the $m_{o}, k$, and $q$ parameters associated with the Drude oscillator, again focusing on monomers. As discussed above, although the electron binding energies depend approximately linearly on $q^{2} / k$, they are not expected to depend sensitively on the choice of $q$ and $k_{s}$ separately or on the choice of $m_{o}$. This was confirmed by exploratory calculations on $\mathrm{HCN}$ and $\mathrm{HNC}$. All results reported here were obtained with the choice $m_{o}=1, q=1$. Because the polarizabilities of $\mathrm{HCN}$ and HNC have considerable anisotropy, we have generalized Eq. (21) to employ separate $k_{z}$ and $k_{x}\left(=k_{y}\right)$ force constants and chose $q^{2} / k_{z}=\alpha_{z z}$ and $q^{2} / k_{x}=q^{2} / k_{y}=\alpha_{x x}$, where the $\alpha_{z z}$ and $\alpha_{x x}$ polarizability components are determined from MP2 calculations. The charges and other parameters used in the model potentials are summarized in Table II.

\section{Extension of the model potential to clusters}

In applying the one-electron model potential approach to clusters of polar molecules, Eqs. (19)-(22) were extended so that the excess electron experiences repulsive, electrostatic, polarization, and dispersion interactions with each monomer. We also allowed for the effects intermonomer polarization. To treat the polarization and dispersion interactions a Drude oscillator was associated with each monomer. To 
illustrate the approach, we consider the specific case of an electron interacting with two monomers, and then demonstrate the method on $(\mathrm{HCN})_{2}$ and $(\mathrm{HNC})_{2}$.

The Hamiltonian for an excess electron interacting with a molecular dimer is:

$$
H=H^{e l}+H^{o s c 1}+H^{o s c 2}+H^{1,2}+H^{\text {couple }, 1}+H^{\text {couple }, 2},
$$

where $H^{e l}$ is the electronic Hamiltonian extended to allow for the electrostatic and repulsive interactions with both monomers, $H^{o s c 1}$ and $H^{o s c 2}$ are the Drude oscillator Hamiltonians associated with the two monomers, $H^{1,2}$ describes the coupling between the two monomers, and $H^{\text {couple, } 1}$ and $H^{\text {couple, } 2}$ describe the coupling of the excess electron with oscillators " 1 " and " 2 ", respectively, with the latter coupling terms being of the form given by Eq. (22). $H^{1,2}$ is given by:

$$
H^{1,2}=q \sum_{i} \frac{Q_{i}^{(1)}}{R_{Q_{i}^{(1)}, 2}^{3}} \mathbf{R}_{Q_{i}^{(1), 2}} \cdot \mathbf{R}_{2}+q \sum_{j} \frac{Q_{j}^{(2)}}{R_{Q_{j}^{(2)}, 1}^{3}} \mathbf{R}_{Q_{j}^{(2), 1}} \cdot \mathbf{R}_{1}+q^{2} \frac{R_{12}^{2} \mathbf{R}_{1} \cdot \mathbf{R}_{2}-3\left(\mathbf{R}_{1} \cdot \mathbf{R}_{12}\right)\left(\mathbf{R}_{2} \cdot \mathbf{R}_{12}\right)}{R_{12}^{5}}
$$

where $Q_{i}^{(\ell)}$ refers to a charge located on monomer 1 , and $\mathbf{R}_{Q_{i}^{(1)}, m}$ is the vector from the $+q$ charge of the Drude oscillator associated with monomer $m$ to the $Q_{i}^{(\ell)}$ charge. $\mathbf{R}_{1}$ and $\mathbf{R}_{2}$ are the vectors associated with the two Drude oscillators, and $\mathbf{R}_{12}$ is the vector between the oscillators. (See Fig. 5 for the definitions of the various vectors.) The first two terms on the right-hand side of the above equation give the interaction between the permanent charges on one monomer and the induced dipole on the other, and the last term gives the interaction between the Drude oscillators and is responsible for the dispersion interaction between the monomers as well as the enhancement of the induced dipole on one monomer due to the presence of the induced dipole on the other monomer. The coupling between the Drude oscillators is ignored in the present treatment, but would be straightforward to include. 
There are several ways of partitioning the full Hamiltonian given by Eq. (33). In this study we use

$$
H^{0}=H^{e l}+\left\langle 0\left|H^{\text {couple }, 1}\right| 0\right\rangle_{o}+\left\langle 0\left|H^{\text {couple }, 2}\right| 0\right\rangle_{o}+H^{\text {osc } 1}+H^{\text {osc } 2}+H^{1,2},
$$

and

$$
V=H^{\text {couple }, 1}+H^{\text {couple }, 2}-\left\langle 0\left|H^{\text {couple }, 1}\right| 0\right\rangle_{o}-\left\langle 0\left|H^{\text {couple }, 2}\right| 0\right\rangle_{o},
$$

where the subscript $o$ on the brackets indicates that the average is over the oscillator functions. The use of $H^{o s c 1}+H^{o s c 2}+H^{1,2}$ as the zeroth-order Hamiltonian for the Drude oscillators results in polarized oscillator wave functions, and the inclusion of the $\left\langle 0\left|H^{\text {couple }, 1}\right| 0\right\rangle_{o}$ and $\left\langle 0\left|H^{\text {couple }, 2}\right| 0\right\rangle_{o}$ terms in $H^{0}$ permits the excess electron to "see" at zeroth-order the induced dipoles resulting from intermonomer polarization.

The basis set used in describing the electron-dimer system consists of products of the form

$$
\psi=\phi_{\alpha} \chi_{i}^{(1)} \chi_{j}^{(2)}
$$

where $\phi_{\alpha}$ is an orbital associated with $H^{e l}$, and $\chi_{i}^{(1)}$ and $\chi_{j}^{(2)}$ are polarized wavefunctions associated with the two Drude oscillators. The geometries used for $(\mathrm{HCN})_{2}$ and $(\mathrm{HNC})_{2}$, in both the model potential and ab initio calculations, were generated by optimizing the intermonomer separation at the MP2/aug-cc-pVTZ level, keeping the monomers rigid. The geometrical parameters are defined in Fig. 6 and their values listed in Table II.

\section{E. Ab initio calculations and choice of basis sets}

To aid in assessing the results of the model potential calculations, all-electron $a b$ initio calculations were carried out at the Hartree-Fock (HF), MP2, and CCSD(T) levels 
of theory for each of the four systems considered. These calculations employed the augcc-pVTZ basis set augmented with six diffuse $s$ and six diffuse $p$ shells on the "terminal" $\mathrm{H}$ atom. The exponents of the supplemental functions were in a geometrical ratio of five and ranged from $5^{*} 10^{-3}$ to $1.6^{*} 10^{-6}$ for the $s$ functions and from $2 * 10^{-2}$ to $6.4^{*} 10^{-6}$ for the $p$ functions. Test calculations revealed that the electron binding energies are essentially unchanged upon further expansion of the $s+p$ space. Inclusion of diffuse $d$ functions was also found to be relatively unimportant, contributing at most a few percent to the electron binding energies. The $a b$ initio calculations were carried out using the Gaussian 98 program. ${ }^{87}$ As will be shown later in the discussion, the agreement between the Drude model electron binding energy and $\operatorname{CCSD}(\mathrm{T})$ electron binding energies and, which is usually used a reference results are not very satisfactory. Intrigue by this finding, Prof. Gutowski carried out CCSDT calculation on HCN and HNC, and found the Drude model actually is giving better total binding energies. The CCSDT binding energies calculated by Prof. Gutowski will also be included in the following discussion.

The one-electron model potential calculations used the same $6 s+6 p$ set of diffuse Gaussians as employed in the $a b$ initio calculations. The basis sets for the Drude oscillators was chosen to consist of the $(l, m, n), 0 \leq l+m+n \leq 2$, harmonic oscillator functions, where the $l, m$, and $n$, indices indicate the level of excitation of the oscillators in the $X, Y$, and $Z$ degrees of freedom respectively.

\section{RESULTS}

In reporting the results we will first consider the monomers followed by the dimers, in each case first reporting the results of the ab initio calculations. For the model- 
potential calculations, results obtained using atomic charges determined at both the HF and $\operatorname{CCSD}(\mathrm{T})$ levels of theory will be presented.

\section{A. Ab initio calculations}

Table III reports $a b$ initio electron binding energies results calculated at the KT, MP2, CCSD(T) and CCSDT levels of theory for HCN and HNC, the KT, MP2 and $\mathrm{CCSD}(\mathrm{T})$ levels of theory for $(\mathrm{HCN})_{2}$ and $(\mathrm{HNC})_{2}$. In addition, polarization contributions, associated with the changes in the electron binding energies in going from the KT to the $\triangle \mathrm{SCF}$ approximation, in which the binding energy is calculated from the difference of the Hartree-Fock energies of the anion and neutral species, are reported. The MP2 contributions to the $a b$ initio electron binding energies are decomposed into dispersion and non-dispersion contributions using a procedure introduced by Gutowski, et al. ${ }^{23,26,70}$ For $\mathrm{HCN}, \mathrm{HNC}$, and $(\mathrm{HCN})_{2}$ our ab initio values for the electron binding energies are very close to those reported previously. ${ }^{26,27} \mathrm{We}$ are unaware of any previous calculations on $(\mathrm{HNC})_{2}^{-}$.

\section{HCN and HNC}

In the $\mathrm{KT}$ approximation the electron binding energies of $\mathrm{HCN}$ and $\mathrm{HNC}$ are predicted to be only -11.7 and $-2.7 \mathrm{~cm}^{-1}$, respectively. The larger (greater in magnitude) $\mathrm{BE}^{\mathrm{KT}}$ value for $\mathrm{HCN}$ is consistent with its larger dipole moment $(3.33$ vs. $2.86 \mathrm{D})$ in the Hartree Fock approximation. For both molecules the polarization contribution to the electron binding energy is much smaller in magnitude than the $\mathrm{BE}^{\mathrm{KT}}$ value. The inclusion of second-order electron correlation effects leads to a much larger change in the electron binding energy of HNC than that of $\mathrm{HCN}\left(-9.1 \mathrm{~cm}^{-1} v s .-0.5 \mathrm{~cm}^{-1}\right)$. The decomposition of the second-order contributions into dispersion and non-dispersion components reveals 
that these two components are comparable in magnitude for both molecules, but the nondispersion component is positive for $\mathrm{HCN}$ and negative for $\mathrm{HNC}$. The dispersion contribution to the electron binding energy is necessarily negative. The small secondorder correction to the electron binding energy of $\mathrm{HCN}$ is thus a result of a near cancellation between the dispersion and non-dispersion contributions. Upon inclusion of electron correlation effects, the dipole moment of $\mathrm{HCN}$ is reduced whereas that of $\mathrm{HNC}$ is increased. Apparently the second-order non-dispersion contribution to the electron binding energy is dominated by the change in the dipole field experienced by the excess electron, and thus is positive for $\mathrm{HCN}$ and negative for $\mathrm{HNC}$.

Higher-order electron correlation effects are predicted to lead to changes of -0.5 and $-23.8 \mathrm{~cm}^{-1}$ in the electron binding energies of $\mathrm{HCN}$ and $\mathrm{HNC}$, respectively. These results are obtained by subtracting the MP2 binding energies from the CCSDT binding energies. The greater importance of higher-order electron correlation effects for the electron-HNC system is primarily due to the enhancement of the dipole moment of the neutral molecule due to electron correlation effects. The best estimate of the electron binding energy for $\mathrm{HCN}\left(-13.2 \mathrm{~cm}^{-1}\right)$ is slightly more than the KT value, but for HNC it is about 12 times larger (at $-35.7 \mathrm{~cm}^{-1}$ ) than the KT value. It is astonishing to see the large difference between CCSD(T) and CCSDT binding energy of HCN and HNC. There is a $-4.2 \mathrm{~cm}^{-1}$ difference between CCSDT and $\operatorname{CCSD}(\mathrm{T})$ binding energy for the electronHCN system, a $5.6 \mathrm{~cm}^{-1}$ difference between CCSDT and CCSD(T) binding energy for the electron-HNC system. For the electron-HCN system, this lead to about $47 \%$ underestimation of electron binding energy at $\operatorname{CCSD}(\mathrm{T})$ level of theory. 


\section{2. $(\mathrm{HCN})_{2}$ and $(\mathrm{HNC})_{2}$}

In the KT approximation the ab initio electron binding energies of $(\mathrm{HCN})_{2}$ and $(\mathrm{HNC})_{2}$ are calculated to be -461 and $-366 \mathrm{~cm}^{-1}$, respectively. These are 39 and 135 times larger in magnitude than the corresponding binding energies of the monomers. Whereas second-order correlation effects lead to a large $-504 \mathrm{~cm}^{-1}$ change in the binding energy of $(\mathrm{HNC})_{2}$, they lead to only a relatively small $-63 \mathrm{~cm}^{-1}$ change in that of $(\mathrm{HCN})_{2}$. As for the monomers, the second-order dispersion and non-dispersion contributions are sizable for both dimers, with the two contributions being of the same sign (negative) for $(\mathrm{HNC})_{2}$ and of opposite sign for $(\mathrm{HCN})_{2}$. Again, the different signs of the non-dispersion contribution to the electron binding for the two dimers can be understood in terms of the changes in the dipole moments upon the inclusion of electron correlation effects, which cause a decrease of the dipole moment of $(\mathrm{HCN})_{2}$ but an enhancement of that of $(\mathrm{HNC})_{2}$. Higher-order correlation effects, when estimated by the difference between the MP2 binding energies and the CCSD(T) binding energies, cause relatively small changes (-1

and $-93 \mathrm{~cm}^{-1}$ for $(\mathrm{HCN})_{2}$ and $(\mathrm{HNC})_{2}$, respectively) in the electron binding energies. CCSDT results for these systems are not available.

\section{B. Model potential calculations}

As noted above, the model potential calculations were carried out using atomic charges determined from HF (model I) as well as from CCSD(T) (model II) calculations. For each choice of charges, electron binding energies are reported at the KT (i.e., neglecting electron-molecule polarization and dispersion interactions), "MP2", and single-plus-double excitation configuration interaction (SDCI) levels of theory. 
For the model potentials described above, the SDCI calculations correspond to full-CI calculations in the sense that all excitations possible with the adopted basis sets are included. However, for the electron/dimer systems, full-CI calculations would also include triple excitations, (i.e., simultaneous excitation of the electron and both Drude oscillators) were the coupling term between the Drude oscillators in Eq. (34) retained.

\section{HCN and HNC}

By design, model I reproduces the ab initio KT binding energies of the monomers (as the repulsive potentials were scaled to give this result). Outside the core regions, the lowest bound orbitals of the model electronic Hamiltonians for $\mathrm{HCN}$ and $\mathrm{HNC}$ are nearly identical to the lowest unoccupied molecular orbitals (LUMOs) from the Hartree-Fock calculations on the neutral molecules. This is illustrated in Fig.7.

Model I gives polarization energies nearly identical to the ab initio values, which is not surprising, as $q^{2} / k_{x}, q^{2} / k_{y}$ and $q^{2} / k_{z}$ were chosen to reproduce the monomer polarizabilities. Most encouragingly, the second-order dispersion contributions to the electron binding energies calculated using model I are close to the ab initio estimates, illustrating the success of the Drude model for treating the dispersion interactions. Nonetheless, the net binding energies calculated through second order using model I differ appreciably from the corresponding ab initio results. In addition, the full-CI binding energies calculated using model I differ appreciably from the results of the allelectron CCSDT calculations, predicting a sizable over-binding for $\mathrm{HCN}$ and an underbinding for HNC. The main reason for this is that model I does not account for the "renormalization" of the dipole moments resulting from inclusion of electron correlation effects and which, as noted above, is primarily responsible for the non-dispersion 
contributions in the ab initio calculations. This problem is remedied in model II, which employs CCSD(T) charges for the monomers.

The charge renormalization built into model II leads to changes in the KT binding energies and in the spatial extent of the zeroth-order wavefunction describing the weakly bound electron. This, in turn, causes changes in the second- and higher-order corrections to the electron binding energies. For both $\mathrm{HCN}$ and $\mathrm{HNC}$, the net electron binding energies calculated through second order using model II are in fairly good agreement with the ab initio MP2 results. Moreover the electron binding energies calculated using the model II full CI method are in good agreement with those calculated using the $a b$ initio CCSDT method. Specifically, for HCN and HNC the full-CI calculations with model II give electron binding energies of -12.3 and $-29.3 \mathrm{~cm}^{-1}$, respectively, as compared to the corresponding ab initio CCSDT values of -13.2 and $-35.7 \mathrm{~cm}^{-1}$. In assessing these results, it should be noted that $\mathrm{HCN}$ and $\mathrm{HNC}$ are undoubtedly two of the more challenging systems for describing the binding of an excess electron by either $a b$ initio or model potential approaches. $\mathrm{HCN}$ is challenging because of its very small $(\approx-$ $13.2 \mathrm{~cm}^{-1}$ ) binding energy and because of the near cancellation between the dispersion and "charge renormalization" contributions to the electron binding energy. HNC represents an even more challenging case in that the inclusion of electron correlation effects leads to about a thirteen-fold increase in the electron binding energy (as evaluated using $a b$ initio calculations). The zeroth-order Hartree-Fock wavefunction is such a poor starting point for these systems that the $\operatorname{CCSD}(\mathrm{T})$ result is not well converged. The model potential approach is providing a more realistic description of electron binding to $\mathrm{HCN}$ and HNC than are the ab initio $\operatorname{CCSD}(\mathrm{T})$ calculations. 


\section{2. $(\mathrm{HCN})_{2}$ and $(\mathrm{HNC})_{2}$}

From the results reported in Table III it is seen that models I and II are less successful for the dimers than the monomers. For example, model I underestimates the KT electron binding energies of the dimers by $14-20 \%$ and gives dispersion contributions to the binding energy about $50 \%$ smaller than the ab initio estimates. The failure of model I to give the correct values of the electron binding energy in the KT approximation has a simple explanation, namely that this model does not reproduce the HF dipole moments of the dimers, in part because of the neglect of the third term on the right hand side of Eq. (34). (The underbinding of the electron at the KT level, in turn, causes the dispersion contributions to be underestimated.) Similarly, model II does not reproduce the $\operatorname{CCSD}(\mathrm{T})$ dipole moments of the dimers. To remedy these deficiencies, models III and IV, in which the atomic charges are scaled so that the net (permanent plus induced) dimer dipole moments agree with the $\mathrm{HF}$ and $\operatorname{CCSD}(\mathrm{T})$ values, respectively, were introduced.

The KT values of the electron binding energies of the dimers calculated using model III are very close to the $a b$ initio KT results. In addition this model gives polarization energies close to the ab initio results. On the other hand, the dispersion contributions to the electron binding energies of the dimers calculated using model III are still considerably (30-39\%) smaller than the corresponding values estimated by partitioning the ab initio MP2 energies. However, the discrepancies between the model potential and ab initio estimates of the dispersion energy should not be ascribed solely to deficiencies in the model potential as the procedure used to decompose the ab initio 
second-order contribution to the electron binding energy into dispersion and nondispersion components is not rigorous and may overestimate dispersion.

Of the four models considered, model IV, which reproduces the CCSD(T) dipole moments of the neutral dimers, should be the most realistic for describing the net electron binding energies of the dimers. Indeed, the electron binding energies calculated through second order using this model $\left(-463\right.$ and $-746 \mathrm{~cm}^{-1}$ for $(\mathrm{HCN})_{2}$ and $(\mathrm{HNC})_{2}$, respectively) are in good agreement with the corresponding ab initio values of -524 and $870 \mathrm{~cm}^{-1}$. The SDCI binding energies calculated using model IV also compare favorably with the ab initio $\mathrm{CCSD}(\mathrm{T})$ results, with the two approaches giving nearly identical electron binding energies for $(\mathrm{HCN})_{2}$, and reasonable, but poorer, agreement for $(\mathrm{HNC})_{2}$ $\left(-792 \mathrm{~cm}^{-1}\right.$ and $-963 \mathrm{~cm}^{-1}$ for the model IV SDCI and ab initio CCSD(T) calculations, respectively). The smaller (in magnitude) electron binding energy of $(\mathrm{HNC})_{2}$ predicted using the model potential in conjunction with the SDCI method compared to that obtained from the $a b$ initio $\operatorname{CCSD}(\mathrm{T})$ calculations is not surprising given that similar behavior was found for the HNC monomer. As demonstrated for the monomer, part of the discrepancy between the model potential and ab initio results for $(\mathrm{HNC})_{2}$ could be due to an inadequacy of the ab initio $\operatorname{CCSD}(\mathrm{T})$ calculations.

The $\mathrm{HCN},(\mathrm{HCN})_{2}, \mathrm{HNC}$ and $(\mathrm{HNC})_{2}$ species considered in this paper all have dipole moments sufficiently large so as to guarantee electron binding. Exploratory calculations reveal that model IV also works well for describing electron binding to clusters of polar molecules with no net dipole moments, e.g., dimers with the dipoles of the monomers opposed (e.g., $\mathrm{NCH} \cdots \mathrm{HCN}$ and $\mathrm{CNH} \cdots \mathrm{HNC})$ in which the excess electron is "localized" between the two dimers. 


\section{CONCLUSION}

It is now well established that the $a b$ initio treatment of the interactions of excess electrons with polar molecules requires very flexible basis sets and the inclusion of highorder correlation effects. This limits such calculations to relatively small cluster systems. One-electron model potential approaches, on the other hand, can be applied to very large clusters. However, for such approaches to reliably describe electron binding, they need to incorporate dispersion interactions between the excess electron and the molecules of the cluster.

In this study a new model potential approach for describing the interaction between an excess electron and polar molecules or clusters of polar molecules was described. The novel feature of this approach is the use of Drude-oscillators to account for polarization and dispersion interactions. The new model potential was tested on the dipole-bound anions of $\mathrm{HCN}, \mathrm{HNC},(\mathrm{HCN})_{2}$ and $(\mathrm{HNC})_{2}$, and was found to give binding energies in good agreement with those from high-level $a b$ initio calculations in all the cases. Especially encouraging is that for $\mathrm{HCN}$ and $\mathrm{HNC}$ the model potential is giving better agreement with $a b$ initio CCSDT results than $a b$ initio $\operatorname{CCSD}(\mathrm{T})$ method does.

There are several potential sources of error in the model potentials used in the present study. These include the approximations in generating the repulsive core, the use of a simple three-point charge model to represent the charge distributions of the monomers, the use of a single polarizable center on each monomer, the neglect of exchange, and the neglect of the hyper- and higher-order polarizabilites. However, none of these approximations is essential, and we envision the model described in this section 
as the starting point for a family of increasingly "sophisticated" model potentials for describing electron-polar molecule interactions.

\section{Negatively charged water clusters}

\section{General Considerations:}

Electron-water systems have long fascinated researchers. Reactions involving electrons in bulk water are of fundamental importance in radiation chemistry, electrochemistry and biology. ${ }^{88-92}$ Yet, even the nature of an excess electron in bulk water remains controversial. ${ }^{93-103}$ Although the long-held view is that an excess electron in bulk water is trapped in an approximately spherical cavity, ${ }^{104-107}$ giving the so-called hydrated electron, Domcke and coworkers have proposed that the excess electron is actually associated with a $\mathrm{H}_{3} \mathrm{O}$ species. ${ }^{100}\left(\mathrm{H}_{2} \mathrm{O}\right)_{n}{ }^{-}$clusters have also received

considerable attention from the experimental and theoretical communities. ${ }^{59,77,108-115}$ There remain several fundamental questions about these species, including the size cluster for which the electron is more stable in an interior rather than a surface state, and how the anions observed experimentally are formed.

$\left(\mathrm{H}_{2} \mathrm{O}\right)_{n}{ }^{-}$clusters were first detected mass spectroscopically by Haberland and coworkers. ${ }^{116}$ Figure 8 reproduces a mass spectrum of $\left(\mathrm{H}_{2} \mathrm{O}\right)_{n}{ }^{-}$clusters from the Johnson group. ${ }^{113}$ This spectrum is noteworthy by the absence of a peak for the monomer, consistent with its dipole moment being too small to support a dipole-bound anion, and by the occurrence of magic numbers at $n=2,6,7$, and 11, and a monotonically decreasing intensity distribution for $n \geq 15$. The origin of the magic numbers in the $\left(\mathrm{H}_{2} \mathrm{O}\right)_{n}{ }^{-}$mass spectra is still a matter of debate, although, there seems to be a correlation 
between the dipole moment of the neutral cluster and the intensity of the peak in the mass spectrum, at least for the smaller clusters. For example, the most stable forms of the $n=$ 2, 6, 7 and 11 clusters, which appear as magic numbers in the anion mass spectrum, have sizable dipole moments, ${ }^{117-119}$ whereas the most stable isomers of the $\left(\mathrm{H}_{2} \mathrm{O}\right)_{n}, n=3-5$, and 8 clusters, for which the corresponding anions are either absent or appear only weakly in the mass spectrum, have zero or small dipole moments. This suggests that the interaction of the excess electron with the dipole field of the neutral clusters may be an important factor in the initial electron capture process. On the other hand, Kim et al. have noted that the $n=10$ cluster also has a sizable $(\approx 2.7 \mathrm{D})$ dipole moment but appears only weakly in the mass spectrum, which would appear to contradict the hypothesis that there is a correlation between the dipole moment and anion formation. ${ }^{118,119}$ However, it is possible that the low yield of $\left(\mathrm{H}_{2} \mathrm{O}\right)_{10}{ }^{-}$in the mass spectrum is the consequence of a relatively low population of $\left(\mathrm{H}_{2} \mathrm{O}\right)_{10}$ in the neutral cluster distribution.

The proceeding discussion assumes that the observed $\left(\mathrm{H}_{2} \mathrm{O}\right)_{n}{ }^{-}$ions result from electron capture by preformed neutral $\left(\mathrm{H}_{2} \mathrm{O}\right)_{n}$ clusters. In the experiments of Johnson et al., electrons are ejected into an expansion containing water and Argon, and the Ar-free $\left(\mathrm{H}_{2} \mathrm{O}\right)_{n}{ }^{-}$clusters are believed to result from electron capture by $\left(\mathrm{H}_{2} \mathrm{O}\right)_{n} \mathrm{Ar}_{m}$ clusters followed by evaporation of Ar atoms. The most abundant isotope of Ar has a mass nearly identical to that of the water dimer, and the weak peaks that appear to correspond to $\left(\mathrm{H}_{2} \mathrm{O}\right)_{4}{ }^{-},\left(\mathrm{H}_{2} \mathrm{O}\right)_{8}{ }^{-}$, and $\left(\mathrm{H}_{2} \mathrm{O}\right)_{9}{ }^{-}$, for the most part, are actually due to $\left(\mathrm{H}_{2} \mathrm{O}\right)_{2}{ }^{-} \mathrm{Ar},\left(\mathrm{H}_{2} \mathrm{O}\right)_{4}{ }^{-} \mathrm{Ar}$ and $\left(\mathrm{H}_{2} \mathrm{O}\right)_{7}{ }^{-} \mathrm{Ar}$, respectively. ${ }^{113}$

Although there is a consensus that the observed forms of $\left(\mathrm{H}_{2} \mathrm{O}\right)_{n}{ }^{-}, n=2-4$, have chain-like structures, the situation regarding the larger clusters is less clear. ${ }^{59,110,120-122}$ 
Neither the mass spectra nor the measured electron detachment energies allow one to definitely establish the geometrical structures. Recently, the Johnson group has succeeded in measuring vibrational spectra in the $\mathrm{OH}$ stretching region for the $\quad\left(\mathrm{H}_{2} \mathrm{O}\right)_{n}{ }^{-} \mathrm{Ar}_{m}, n=5$ 9, clusters. ${ }^{58,59,123}$ The presence of attached Ar atoms assures that the clusters are cold ( $\mathrm{T}$ $\preccurlyeq 50 \mathrm{~K})$. The comparison of measured spectra with ab initio calculated spectra for various isomers of the bare (i.e., non-Ar solvated) clusters greatly narrows down the possible structures. However, even with the availability of the IR data, the structures of the $\left(\mathrm{H}_{2} \mathrm{O}\right)_{n}{ }^{-}, n \geq 5$, clusters remain subject to debate. For example, whereas Ayotte et al. ${ }^{59}$ have proposed that the observed IR spectra of $\left(\mathrm{H}_{2} \mathrm{O}\right)_{6}{ }^{-}$and it deuterium substituted isotopomers are due to a chain-like isomer, Kim and coworkers have suggested a more three-dimensional structure as the origin of the spectrum. ${ }^{10,120,122}$ One might expected that comparison of the experimental IR spectrum with the calculated spectra for different isomers would permit definitive assignment for a cluster the size of $\left(\mathrm{H}_{2} \mathrm{O}\right)_{6}{ }_{6}^{-}$. However, due to the need to employ large, flexible basis sets, is has been necessary to adopt either density functional theory (e.g., Becke3LYP ${ }^{124-126}$ ) or the MP2 method for calculating the frequencies. Since the Becke3LYP method considerably overbinds the excess electron, while the MP2 procedure considerably underbinds it and since the vibrational frequencies and intensities depend on the degree of localization of the excess electron, neither of these methods can account for the observed IR spectrum in a quantitative manner. ${ }^{59}$ Density functional methods also suffer from the inability to treat long-range dispersion interactions. ${ }^{127}$ The remedy, namely to calculate the vibrational spectrum at the $\operatorname{CCSD}(\mathrm{T})$ level, is too computationally demanding at present. This signals the need for 
alternative theoretical approaches to treat the anion states of clusters of water and of other polar molecules.

Models for including dispersion interaction between an excess electron interacting with a cluster of polar molecules have been introduced by two groups. The Drude-model approach presented above is due to our group. Sindelka et al. ${ }^{36}$ have also recently introduced a model for calculating second-order dispersion interactions between an excess electron and a polar molecule or cluster of polar molecules. The main difference is that while Eq. 24 employs a discretization of the continuum, Sindelka et al. calculated the dispersion energy by doing an integral over the continuum. A second difference is that we have damped the short-range coupling between the electron and the polarizable monomers, while Sindelka et al. used an undamped interaction. However, this damping is relatively unimportant for the second-order energies. The two approaches also differ in terms of the choice of excitation energy associated with the monomer. However, as argued above, the dispersion energy is relative insensitive to the monomer excitation energy.

\section{Applications of the Drude model to water clusters}

We now consider in more detail the use of one-electron model potentials for describing the interaction of an excess electron with water. Water has been chosen for focus because of the large body of experimental and theoretical work done on excess electrons interacting with water in clusters, films, and the bulk, and because there are several unresolved issues concerning electron-water systems that can not be answered by current ab initio methods. 
There is a rich history of one-electron model approaches to treating electron-water systems. In particular, we note the pioneering work of Landman and co-workers, ${ }^{74}$ Rossky et al., ${ }^{75}$ Staib and Borgis, ${ }^{76}$ and Berne and co-workers. ${ }^{77}$ The key features of the models introduced by these researchers, as well as of a more recent model of Mosyak et al., ${ }^{81}$ are summarized in Table IV. These models have been used to address a variety of problems, including estimating the size water cluster for which it is more favorable for an excess electron to be bound in the interior as opposed to the surface, ${ }^{128-130}$ calculating electron transport through water films, ${ }^{131}$ and simulating the dynamics of the solvated electron following photo-excitation. ${ }^{132,133}$ Most of these one-electron models allow for polarization of the water molecules by the excess electron, but none allow for dispersion interactions between the excess electron and the water molecules. As is clear from Table I, the dispersion contribution to the electron binding energy is typically an order of magnitude more important than polarization (at least for small clusters) and it is generally comparable to the electrostatic binding energies. This raises the possibility that dispersion interactions could significantly affect the dynamics of solvated electrons or impact the preference for electron binding in the interior $v$ s. the surface of a cluster.

In this work, the Dang-Chang (DC) polarizable model for water, ${ }^{134}$ which has been found to do a good job at describing small water clusters and bulk water, ${ }^{134,135}$ is combined with the Drude model described in section 3. The unique feature of the current model is the use of Drude oscillators to treat electron-water monomer polarization and dispersion. The resulting one-electron model potential approach is applied to $\left(\mathrm{H}_{2} \mathrm{O}\right)_{3}{ }^{-}$and $\left(\mathrm{H}_{2} \mathrm{O}\right)_{4}{ }^{-}$, and the results are compared with the predictions of ab initio CCSD(T) 
calculations. The computational details are described in Section III, with the results being presented in Section IV, followed by Discussion and Conclusions sections.

\section{COMPUTATIONAL METHODOLOGY}

\section{A. Model Potential}

The DC water model employs the experimental geometry of the gas phase monomer $\left(\mathrm{ROH}=0.9572 \AA, \mathrm{HOH}\right.$ angle $\left.=104.52^{\circ}\right)$ and is a rigid-monomer model. Point charges are located at the positions of the $\mathrm{H}$ atom $(\mathrm{Q}=0.519 \mathrm{e})$ and on the rotational axis, displaced $0.215 \AA$ from the $\mathrm{O}$ atom (towards the $\mathrm{H}$ atom) $(\mathrm{Q}=-1.038 \mathrm{e})$. This gives

a dipole moment of $1.848 \mathrm{D}$, nearly identical to the experimental value of $1.825 \mathrm{D}^{136}$ and quadruple moment components of $\mathrm{Q}_{x x}=2.235 \mathrm{D} \cdot \AA \mathrm{Q}_{\mathrm{yy}}=-2.047 \mathrm{D} \cdot \AA$, and $\mathrm{Q}_{z z}=-$ $0.188 \mathrm{D} \cdot \AA$ in fairly good agreement with experiment values of $\mathrm{Q}_{\mathrm{xx}}=2.626 \mathrm{D} \cdot \AA, \mathrm{Q}_{\mathrm{yy}}=$ $-2.493 \mathrm{D} \cdot \AA$, and $\mathrm{Q}_{\mathrm{zz}}=-0.134 \mathrm{D} \cdot \AA^{137}{ }^{137}$ The DC model also locates a polarizable center at the same position as the negative charge. The polarizability is taken to be isotopic, with the numerical value of $1.444 \AA^{3}$, chosen to match the experiment. ${ }^{138}$ Finally; a single Lennard-Jones site is centered on the $\mathrm{O}$ atom $(\epsilon=0.1825 \mathrm{kcal} / \mathrm{mol}, \sigma=3.2340 \AA)$.

The water-water interactions thus include electrostatic (charge-charge), chargeinduced dipole, and Lennard-Jones type interactions. The induced dipoles on the different monomers are allowed to interact, and the polarization equations given the individual induced dipole moments are solved self consistently.

The Hamiltonian (in atomic units) for an excess electron interacting with a single water molecule and neglecting polarization and dispersion is taken to be 


$$
H_{D C}^{e l}=-\frac{1}{2} \nabla^{2}-\sum_{j} \frac{Q_{j}}{r_{j}}+V^{e x}+V^{r e p}
$$

where the sum is over the charge sites of the monomer, and $V^{e x}$ and $V^{\text {rep }}$ represent the exchange and electron-molecule repulsive interactions, respectively. The subscript "DC" implies that the charges and their locations are from the DC model. As will be discussed below, we also consider a second model based on Hartree Fock (HF) charges in which case the electronic Hamiltonian is designated $H_{H F}^{e l} \cdot V^{\text {rep }}$ is generated following the procedure of Schnitker and Rossky, ${ }^{75}$ except that we represent it in terms of Gaussian instead of Slater functions. Also, following Schnitker and Rossky, $V^{e x}$ is ignored as it is expected to be relatively unimportant for the electron binding to water clusters.

Electron-molecule polarization and dispersion are accounted for by associating with the monomer a Drude oscillator consisting of two charges $+q,-q$, separated by a distance $R$ and coupled harmonically through the force constant $k$. The reduced mass of the oscillator is $m_{o}$. The Drude oscillator is located at the same position as the polarizable center in the DC model. This results in a coupling term of the form

$$
V^{\text {couple }}=\frac{q \mathbf{r} \cdot \mathbf{R}}{r^{3}} \cdot\left(1-e^{-b r^{2}}\right)
$$

where $\mathbf{r}$ is the position of the electron relative to the oscillator, and $\left(1-e^{-b r^{2}}\right)$ is a damping factor, which attenuates unphysical short-range interactions between the excess electron and the Drude oscillator. The total Hamiltonian then becomes

$$
H=H^{e l}+H^{\text {osc }}+V^{\text {couple }} .
$$

Both the Drude oscillator and the excess electron are treated quantum mechanically using a product basis set of the form $\left|\phi_{\beta} \chi_{i}\right\rangle$ where $\phi_{\beta}$ and $\chi_{i}$ are eigenfunctions of $H^{e l}$ and 
$H^{o s c}$, respectively. The electron binding energy for the full model Hamiltonian is calculated using the second-order perturbation theory and the configuration interaction (CI) methods. In addition, electron binding energies are also calculated using $H^{e l}$ alone, i.e., neglecting coupling to the Drude oscillators. The latter binding energies are refered to as Koopmans' theorem $(\mathrm{KT})^{67}$ values, since they result from a static approximation neglecting polarization and dispersion.

As shown in Reference 33, the second-order correction to the electron binding energy for the model Hamiltonian system can be decomposed into polarization and dispersion contributions. The former is proportional to $q^{2} / k$, which is simply the polarizability of the Drude oscillator, here set equal to either the HF or DC polarizability values of a water monomer. (Anisotropic polarizabilities can be accounted for by introducing separate $\mathrm{k}_{\mathrm{x}}, \mathrm{k}_{\mathrm{y}}$ and $\mathrm{k}_{\mathrm{z}}$ force constants.) The second-order dispersion contribution is:

$$
\varepsilon^{\text {disp }}=\sum_{s} \sum_{\beta \neq 0} \frac{\frac{q^{2}}{2 k}\left|\left\langle 0\left|\frac{s}{r^{3}}\right| \beta\right\rangle\right|^{2}}{-1+\left(\varepsilon_{0}-\varepsilon_{\beta}\right) \sqrt{\frac{m_{o}}{k}}}
$$

where the first summation runs over $s=\mathrm{x}, \mathrm{y}$, and $\mathrm{z}$, and the second runs over the excited electronic levels. However, since the excess electron is weakly bound, the sum over $\beta$ is dominated by terms for which $\left|\left(\varepsilon_{0}-\varepsilon_{\beta}\right) \sqrt{\frac{m_{o}}{k}}\right|<<1$, the dependence on $m_{o}$ should be quite weak. In this work, $m_{o}$ is taken to be the mass of an electron, and $q$ is taken to be 1 . Note that if the excess electron were highly localized, Eq. 41 would reduce to the London expression for dispersion. 
The extension of the Drude model to a cluster of molecules is straightforward. The Hamiltonian for the excess electron now includes electrostatic and repulsive interactions with each monomer, as well as interactions with a Drude oscillator on each molecule. The basis set for an electron interacting with $n$ Drude oscillators consists of functions of the form $\left|\phi_{\alpha} \chi_{l}^{(1)} \cdots \chi_{m}^{(n)}\right\rangle$ where $\phi_{\alpha}$ is again an electron orbital and $\chi_{l}^{j}$ is the $l^{\text {th }}$ level associated with the $j^{\text {th }}$ oscillator.

Intermolecular induction and polarization can be treated either through the standard approach for the DC model or via coupling between the Drude oscillators. The latter approach has the advantage of including, in a self-consistent manner, three- and higher-body induction and dispersion effects, both among the monomers as well as in the electron and monomer interactions. In the present study, we adopt an intermediate approach, in which the induction is treated using Drude oscillators but the intermolecular dispersion is accounted for via the Lennard-Jones terms in the DC water model. This decouples electron-molecule and molecule-molecule dispersion, which should be a good approximation as three-body dispersion effects are expected to be relatively unimportant for water clusters.

In applying the model Hamiltonian approach to clusters, we incorporate into the zeroth-order electronic Hamiltonian the interaction of the excess electron with the induced dipoles on the monomers. That is, the zeroth-order energy levels of the excess electron are calculated in the field of the charges plus the induced dipole moments.

The electronic basis set is chosen to consist of even-tempered series of $s$ and $p$ primitive Gaussian-type functions, the number and exponents of which depend on the cluster being treated. Additional details are provided below after the specific water 
clusters to be studied are introduced. A basis set of the form $\left(\psi_{l}, \psi_{m}, \psi_{n}\right), 0 \leq l+m+n \leq$ 2 , where $l, m$, and $n$ are quantum numbers associated with a three-dimensional harmonic oscillator in Cartesian representation, is employed on each Drude oscillator. Test calculations show that this oscillator basis set is adequate for describing electron-water polarization and dispersion as well as intermolecular induction.

Although the ultimate goal of the model potential calculations is to provide a quantitatively correct description of electron binding to water clusters, it is important to check that the model employed correctly describes electron binding in the absence of electron-water polarization and dispersion. However, one cannot simply compare the electron binding energies calculated using $H_{D C}^{e l}$, described above, with ab initio Koopmans' therorem (KT) binding energies. The reason for this is that $H_{D C}^{e l}$ employs charges that give the correct dipole moment and realistic quadruple moment components, whereas $a b$ initio KT binding energies are obtained using the Hatree-Fock (HF) procedure, which considerably overestimates the dipole moment for the water monomer. Thus to facilitate comparison, we have also carried out model potential calculations with the DC charges replaced by charges determined from Hartree-Fock electrostatic potential calculations on the water monomer. Even though this set of calculations using $H_{H F}^{e l}$ neglects electron-water polarization and dispersion, it is still necessary to include intramolecular polarization as this is included in the $a b$ initio Hartree-Fock calculations. This is accomplished by choosing $q^{2} / k$ to reproduce the Hartree-Fock polarizability of a water monomer and allowing the permanent charges in the $H_{H F}^{e l}$ model to interact with the Drude oscillators. 
In our earlier work on $(\mathrm{HCN})_{\mathrm{n}}^{-}$and $(\mathrm{HNC})_{\mathrm{n}}^{-}, n=1,2$, it was found that the oneelectron model, with charges and polarizabilities from Hartree-Fock calculations, and with the repulsive potential derived using the procedure described in Reference 75, gave electron binding energies considerably lower in magnitude than the ab initio KT values. This was primarily a consequence of the approximations used in the generation of the repulsive core. This problem was solved by rescaling the repulsive potential so that the electron binding energies for the $\mathrm{HCN}$ and $\mathrm{HNC}$ monomers calculated using the model potential procedure reproduced the ab initio KT binding energies. The scaling factors obtained for the monomers also proved satisfactory for the dimers. Moreover, with this rescaling, the model potential method including coupling between the excess electrons and the Drude oscillators was found to give polarization and dispersion contributions to the electron binding energies close to the ab initio results.

For water clusters, it is necessary to modify this strategy somewhat because the monomer does not bind an electron. (Actually, in the Born-Oppenheimer approximation, a water monomer does weakly bind an electron. However, were corrections to the BornOppenheimer approximation made, the excess electron would no longer bind.) For that reason, we have used the water dimer, which does have a bound anion state, to determine the scaling factor for the repulsive potential. This scaling factor is used in all subsequent calculations of electron water interaction, including those discussed below based on the model potential employing DC charges and polarizability values.

The calculations on the water dimer were also used to determine the value of $b$ in the factor damping the coupling of the electron to the Drude oscillators. Specifically, $b$ 
was chosen so that the model potential CI binding energy reproduced the ab initio $\operatorname{CCSD}(\mathrm{T})$ result. This gave a $b$ value of 0.43 .

\section{B. Details of $\left(\mathrm{H}_{2} \mathrm{O}\right)_{3}{ }^{-}$and $\left(\mathrm{H}_{2} \mathrm{O}\right)_{4}^{-}$calculations}

In this work, we consider both cyclic chain-like and crown-like local minima of the $\left(\mathrm{H}_{2} \mathrm{O}\right)_{3}{ }^{-}$and $\left(\mathrm{H}_{2} \mathrm{O}\right)_{4}{ }^{-}$clusters (see Figure 9). In the crown-like structures, the free $\mathrm{OH}$ groups all point in the same direction. The neutral $\left(\mathrm{H}_{2} \mathrm{O}\right)_{3}$ and $\left(\mathrm{H}_{2} \mathrm{O}\right)_{4}$ clusters, at the optimized structures of the anions, have large dipole moments, and thus the excess

electron can be viewed as being dipole-bound. ${ }^{21-24,68}$ Interestingly, for the neutral clusters, neither the chain-like nor the crown-like structures are potential energy minima. Thus the binding of the excess electron overcomes the tendency of these neutral clusters to rearrange. For $\left(\mathrm{H}_{2} \mathrm{O}\right)_{3}{ }^{-}$we also present results for the transition state for interconversion of the chain-like and crown-like structures. ${ }^{139}$

We now turn to the electronic basis sets used in the model potential and ab initio calculations. In the model potential calculations, the basis set consisted of two $s$ Gaussian functions with exponents 0.02526 and 0.1027 and two $p$ functions with exponents 0.141 and 0.727 on each $\mathrm{H}$ atom ${ }^{140}$ together with a large set of diffuse $s$ and $p$ Gaussian functions on the $\mathrm{O}$ atom on the terminal water (at the + dipole end) in the chain like structures and on the rotational axis for the crown-like clusters. In the latter case the displacement of the center of the diffuse functions from the plane of the $\mathrm{O}$ atoms was optimized so as to maximize the electron binding energies. For the model potential calculations on $\left(\mathrm{H}_{2} \mathrm{O}\right)_{3}{ }^{-}$, the large, single-center portion of the basis set was chosen to consist of an even-tempered series of six $s$ and five $p$ functions. For the calculations on the crown-like structure of $\left(\mathrm{H}_{2} \mathrm{O}\right)_{4}{ }^{-}$, five $s$ and four $p$ functions were used, whereas for 
the linear form of $\left(\mathrm{H}_{2} \mathrm{O}\right)_{4}{ }^{-}$, four $s$ and three $p$ functions were used. (For species with a large dipole moment the dipole bound electron is more localized, and a smaller basis set can be employed.) The exponents of the basis functions are listed in Table V. Test calculations revealed that the adoption of still more flexible basis sets had very little effect on the electron binding energies.

The $a b$ initio calculations employed a flexible basis set suitable for describing the neutral water molecules together with the same single-center sets of diffuse $s$ and $p$ functions as used for the model potential calculations. A (11s6p2d/6s $2 p) \rightarrow$ $[5 s 4 p 2 d / 4 s 2 p]$ contracted Gaussian basis set was first adopted for the water molecules. (The uncontracted basis set is given in parentheses and the contracted set in brackets. The $\mathrm{O}$ atom basis set is specified first, followed by the $\mathrm{H}$ atom basis set.) For the $\mathrm{O}$ atoms, the $s$ and $p$ functions were taken from the aug-cc-pVTZ basis set and the $d$ functions from the aug-cc-pVDZ basis set. ${ }^{83}$ Similarly for the $\mathrm{H}$ atoms, the basis set was formed by combining the $s$ functions from the aug-cc-pVTZ basis set with the $p$ functions from the aug-cc-pVDZ basis set. This mixed basis set was found to give at the MP2 level a dipole moment and polarizability of the water monomer close to the corresponding experimental values, while being less computationally demanding than the full aug-cc-pVTZ basis set. The large sets of supplemental diffuse functions are centered in the same locations as in the model potential calculations.

Ab initio results are reported at the KT, MP2, and $\mathrm{CCSD}(\mathrm{T})$ levels of theories. For the last two methods the electron binding energies are obtained by subtracting the energy of the neutral from that of the anion at the specified level of theory. The second-order contributions to the electron binding energies are divided into dispersion and non- 
dispersion components using the procedure of Gutowski et al. ${ }^{23,26,70}$ This procedure associates the dispersion contribution with those terms in the expression for the secondorder correlation energy of the anion that involve simultaneous excitations from the dipole-bound orbital and one of the occupied orbitals of the water cluster.

To facilitate comparison of the model potential and $a b$ initio results, the same geometries were used for the two sets of calculations. The geometries of the anions were optimized at the ab initio MP2 level of theory under the constraint of rigid monomers, using the $\mathrm{OH}$ bond length and $\mathrm{HOH}$ angle values employed in the DC model. These geometries were then used for the ab initio calculations on the neutral and anionic species and for the model potential calculations on the anions.

\section{RESULTS}

\section{A. $\left(\mathrm{H}_{2} \mathrm{O}\right)_{2}^{-}$}

Table VI summarizes the results for $\left(\mathrm{H}_{2} \mathrm{O}\right)_{2}{ }^{-}$. By construction, the KT binding energy from the model potential employing the HF charges and polarizabilities, (model I) matches the ab initio KT value of $11.1 \mathrm{meV}$, and CI result from the model based on $H_{D C}^{e l}$ (model II) reproduces the ab initio $\mathrm{CCSD}(\mathrm{T})$ binding energy of $33.0 \mathrm{meV}$. Experimental estimates of the vertical detachment energy of $\left(\mathrm{H}_{2} \mathrm{O}\right)_{2}{ }^{-}$are $30 \pm 4 \mathrm{meV}$ and $45 \pm 6$ meV. ${ }^{109,141}$

It should be noted that the DC model tends to underestimate the induced dipole moments of water clusters. For example, for the dimer, with the geometry employed here, the DC model gives a net dipole moment of $4.02 \mathrm{D}$, compared with the dipole moment of 4.13 D obtained from ab initio MP2 calculations; for the chain-like form of the tetramer, 
the discrepancy is $0.29 \mathrm{D}$. In general, the underestimation of the dipole moments would be expected to lead to an underestimation of the electron binding energies. However, by choosing the scaling factor of the repulsive potential so that the KT binding energy calculated using model I reproduces the ab initio KT electron binding energy and the damping factor $b$ so that the CI binding energy calculated using model II reproduces the ab initio $\operatorname{CCSD}(\mathrm{T})$ binding energy of the water dimer, we partially correct for this shortcoming of the DC model.

\section{B. $\left(\mathrm{H}_{2} \mathrm{O}\right)_{3}{ }^{-}$}

Table VII reports for $\left(\mathrm{H}_{2} \mathrm{O}\right)_{3}{ }^{-}$the electron binding energies obtained using models I and II and from the $a b$ initio calculations. We consider first the results of the $a b$ initio calculations. In the KT approximation the excess electron is bound by 3.3 and 66.7 $\mathrm{meV}$ in the crown-like and chain-like forms of $\left(\mathrm{H}_{2} \mathrm{O}\right)_{3}$, respectively. The corresponding binding energies obtained from the $\operatorname{CCSD}(\mathrm{T})$ calculations are 13.0 and $127.0 \mathrm{meV}$. The MP2 binding energies are roughly intermediate between the KT and $\operatorname{CCSD}(\mathrm{T})$ values. As has been found for other dipole-bound anions, polarization (estimated by the difference between the KT and $\triangle \mathrm{SCF}$ binding energies) makes relatively small contributions to the electron binding energies. ${ }^{24}$ The decomposition procedure of Gutowski et al. gives second-order dispersion contributions to the electron binding energies of 4.0 and 43.8 $\mathrm{meV}$ for the crown-like and chain-like forms of $\left(\mathrm{H}_{2} \mathrm{O}\right)_{3}$, respectively. These dispersion contributions are roughly comparable to the KT binding energies. The non-dispersion contributions to the electron binding energies act so as to reduce the binding energies by 1.7 and $11.8 \mathrm{meV}$ for the crown- and chain-like structures, respectively. This is primarily 
a consequence of the reduction of the monomer dipole moments brought about by inclusion of electron correlation effects.

The one-electron model-potential approach employing HF charges and the HF polarizability for the monomers (model I) gives KT binding energies very close to the corresponding ab initio values. The polarization and dispersion contributions calculated using model I are also in excellent agreement with the ab initio results.

The net electron binding energies calculated through second-order using model I are considerably (8-34\%) larger than the ab initio MP2 results. This is largely due to the use of HF atomic charges in model I, whereas the ab initio MP2 calculations build in a charge renormalization, which causes a reduction of the dipole moments of the water monomers.

We now examine the results obtained using the one-electron model potential with the DC charges and polarizability (model II). Most significantly, model II gives electron binding energies at the $\mathrm{CI}$ level very close to the $a b$ initio $\operatorname{CCSD}(\mathrm{T})$ results. For the chain-like form of $\left(\mathrm{H}_{2} \mathrm{O}\right)_{3}{ }^{-}$, the ab initio $\mathrm{CCSD}(\mathrm{T})$ and model potential calculations give electron binding energies of 127.0 and $128.6 \mathrm{meV}$, respectively. The corresponding results for the crown-like form of $\left(\mathrm{H}_{2} \mathrm{O}\right)_{3}{ }^{-}$are 13.0 and $13.6 \mathrm{meV}$. The experimental value of the vertical electron detachment energy of $\left(\mathrm{H}_{2} \mathrm{O}\right)_{3}{ }^{-}$(presumably with a chain-like geometry) is $150 \pm 15 \mathrm{meV},{ }^{142}$ which is somewhat larger than the model potential CI and ab initio $\operatorname{CCSD}(\mathrm{T})$ values for the chain-like form of the anion. This discrepancy is primarily a consequence of our use of rigid-monomers in both the model potential and $a b$ initio calculations, which leads to an underestimation of the dipole moment of the neutral molecule. 
Table VII also reports results for the transition state for interconversion of the crown-like and chain-like isomers of $\left(\mathrm{H}_{2} \mathrm{O}\right)_{3}{ }^{-}$. The electron binding energy for this transition state is intermediate between that of the two minimum energy structures. This is consistent with the trends in the dipole moments of the corresponding neutral clusters. Again there is excellent agreement between the electron binding energy from model II CI calculations and that from the ab initio $\operatorname{CCSD}(\mathrm{T})$ calculations.

\section{C. $\left(\mathrm{H}_{2} \mathrm{O}\right)_{4}^{-}$}

Table VIII reports for $\left(\mathrm{H}_{2} \mathrm{O}\right)_{4}{ }^{-}$the electron binding energies obtained using models I and II and from the ab initio calculations. The excess electron is bound much more strongly in the $\left(\mathrm{H}_{2} \mathrm{O}\right)_{4}{ }^{-}$isomers than in their $\left(\mathrm{H}_{2} \mathrm{O}\right)_{3}{ }^{-}$counterparts, consistent with the larger dipole moments for the neutral $\left(\mathrm{H}_{2} \mathrm{O}\right)_{4}$ than $\left(\mathrm{H}_{2} \mathrm{O}\right)_{3}$ clusters. At the ab initio KT level, the crown and chain-like forms of $\left(\mathrm{H}_{2} \mathrm{O}\right)_{4}{ }^{-}$are bound by 12.5 and $110.2 \mathrm{meV}$, respectively. The corresponding values at the MP2 level are 20.9 and $170.3 \mathrm{meV}$, and at the CCSD(T) level, they are 36.5 and $209.7 \mathrm{meV}$. The decomposition procedure of Gutowski et al. again reveals that there are large dispersion and non-dispersion contributions to the electron binding energies which act in opposite directions. However, because the dispersion contributions are larger in magnitude, there is a net enhancement of the electron binding energies upon inclusion of second-order correlation effects.

Model I gives KT binding energies and second-order dispersion contributions to the binding energies close to the $a b$ initio results. As expected, MP2 calculations with model I overestimate the electron binding energies as compared to the ab initio MP2 results. 
The CI calculations with model II give electron binding energies for the crownlike and chain-like forms of $\left(\mathrm{H}_{2} \mathrm{O}\right)_{4}{ }^{-}$very close to the $a b$ initio $\mathrm{CCSD}(\mathrm{T})$ values. Specifically the model potential calculations give electron binding energies of 40.0 and $212.5 \mathrm{meV}$ for the crown-like and chain-like isomers, respectively, whereas the corresponding ab initio CCSD(T) values are 36.5 and $209.7 \mathrm{meV}$. The agreement is better than might have been expected, especially for the chain-like structure, given the considerable $(\approx 0.29 \mathrm{D})$ underestimation of the net dipole moment of the neutral cluster by the DC model. It may be that the error due to the underestimation of the dipole moment is offset by slightly too weak a damping of the coupling of the electron to the Drude oscillator.

We are unaware of an experimental measurement of the vertical detachment energy for the chain-like form of $\left(\mathrm{H}_{2} \mathrm{O}\right)_{4}{ }^{-}$. However, we anticipate that the present calculations (both $\mathrm{CCSD}(\mathrm{T})$ ab initio and CI model potential) give the electron binding energies $10-20 \%$ smaller then the "true" value due to the use of rigid monomer approximation.

\section{Electron densities and importance of single excitations.}

Figure 10 reports the charge distribution of the crown-like form of $\left(\mathrm{H}_{2} \mathrm{O}\right)_{4}{ }^{-}$ obtained using the MP2 and CI methods in conjunction with model II. The charge distribution obtained in the KT approximation and using model II is nearly identical to that obtained using the MP2 method, and thus has not been plotted. The similar charge densities obtained from the KT and MP2 approximations is somewhat surprising given the sizable enhancement in electron binding energy in going from the KT to the MP2 approximation. However, this is consistent with the observation that the wavefunction 
through first order is dominated by the reference configuration, the coefficient of which is 0.998 in the normalized wavefunction. Hence, even though dispersion interactions make a sizable contribution to the electron binding energy, they have very little impact on the distribution of the excess electron.

In contrast to the relative insensitivity of the distribution of the excess electron to first-order corrections to the wavefunction, there is a large difference between the charge distributions obtained from the MP2 and full CI model potential calculations, with the charge distribution associated with the full CI wavefunction being much more localized. The coefficient of the reference configuration in the CI wavefunction for the crown-like form of $\left(\mathrm{H}_{2} \mathrm{O}\right)_{4}{ }^{-}$is only 0.82 .

The large change in the wavefunction (and in the electron binding energy) in going from the MP2 to the CI method is due primarily to configurations in which the electron is excited but the Drude oscillators are unexcited. In a perturbative treatment, these single excitations first enter the wavefunction in second-order and the energy in fourth-order, and they be thought of as a renormalization in response to the dispersion interactions. The reason that these single excitations prove so important is apparent from examination of the perturbative expressions, from which it is seen that they enter with factors of $\varepsilon_{0}-\varepsilon_{j}$ or $\varepsilon_{i}-\varepsilon_{j}$, where $\varepsilon_{0}$ and $\varepsilon_{j}$ are energies associated with $H^{e l}$, in the energy denominators. In contrast, the terms involving excitation of both the electron and a Drude oscillator involve factors in the denominator of the form $\varepsilon_{0}-\varepsilon_{j}+\varepsilon_{D}$, where $\varepsilon_{D}$ is the energy spacing between levels of the Drude oscillator. Since the energy levels of the excess electron are closely spaced compared to those of the Drude oscillator, configurations in which the electron is excited but the Drude oscillators are unexcited 
lead to sizable corrections to the wavefunction and the electron binding energy. The sizable high-order corrections to the electron binding energies of dipole-based anions found in all-electron $a b$ initio calculations ${ }^{23,24,26,27,68}$ have a similar origin, i.e., they arise from configurations, in which the excess electron is excited but the electrons of the molecule or cluster are unexcited.

Figure 11 displays the charge distribution of the excess electron of the chain-like form of $\left(\mathrm{H}_{2} \mathrm{O}\right)_{4}{ }^{-}$calculated using the MP2 and CI methods in conjuction with model II. As for the crown-like anion, the KT calculations using model II give nearly the same electron distribution as obtained from the MP2 calculations, whereas there is a considerable contraction of charge density upon inclusion of configurations involving excitation of the electron only

\section{E. Renormalized MP2-level model potential}

Because our long-range goal is to apply the one-electron Drude model to describe electron binding to large water clusters, the computational speed of the calculations is of importance. This leads us to ask whether the MP2 method can be "renormalized" to give near CI quality results. Here we consider a crude renormalization in which the magnitudes of the permanent charges in model II have been scaled so that for $\left(\mathrm{H}_{2} \mathrm{O}\right)_{2}{ }^{-}$ MP2 calculations approximately reproduce the electron binding energy from the ab initio $\operatorname{CCSD}(\mathrm{T})$ calculations. This renormalized model (hereafter designated as model III) underestimates the electron binding energies of the crown structures (by up to 20\%) and overestimates the electron binding energies of the chain-like structures by up to $8 \%$ (see Table IX). Moreover, it gives electron distributions (Fig. 12 and Fig. 13) that are too extended (i.e., diffuse), compared to those from the CI calculations and using model II. 
Nonetheless, model III should be useful for qualitative (even semi-quantitative) prediction of the electron binding energies of large water clusters.

\section{DISCUSSION AND CONCLUSIONS}

The present study shows that the Dang-Chang water model, coupled with a oneelectron model potential employing Drude oscillators to describe electron-molecule polarization and dispersion, is able to account in a near quantitative manner for the binding of an excess electron to small water clusters. Dispersion and high-order "renormalization" corrections lead to large enhancements of the electron binding energies compared to those predicted from a "purely" electrostatic model neglecting dispersion interactions. Configurations in which the excess electron is excited but the Drude oscillators are unexcited are found cause sizable enhancements of the electron binding energies and significant contraction of the charge distributions of the excess electron. This contraction greatly increases the charge density near the $\mathrm{H}$ atoms near the end of the chains in the chain-like isomers and in the vicinity of the free $\mathrm{OH}$ groups in the crownlike isomers. This, in turn, should prove important for the intensities of the $\mathrm{OH}$ stretching vibrations in IR spectroscopy of the anion and also for the presence of the $\mathrm{OH}$ stretching vibrational structure in the detachment spectra.

For comparison with experimental electron detachment energies and for calculating vibrational spectra, it will be necessary to extend the present model to allow for flexible monomers. This flexibility is especially important for the electron binding energies as both the permanent and induced dipoles associated with the monomers change with distortions of the monomers. 
Although the present investigation has focused on dipole-bound anions of water clusters, the Drude-model approach should also be suited for describing electron binding to mixed clusters containing polar molecules and other atoms or molecules that do not have low-lying valence-type anion states. For example, the Drude model has recently been applied to $\left(\mathrm{H}_{2} \mathrm{O}\right)_{2}{ }^{-} \mathrm{Ar}_{m}, m \leq 12$, clusters, again, using a one-electron Hamiltonian, but with polarization and dispersion interactions involving the Ar atoms as well as the water molecules being described by Drude oscillators. ${ }^{143}$ These calculations reveal that the variation in the electron binding energies caused by the clustering with Ar atoms is the result of an interplay of several factors, including, changed electrostatics due to the induced dipoles on the Ar atoms, an "excluded-volume" effect due to the repulsive potential on the Ar atoms, and polarization and dispersion interactions between the excess electron and the Ar atoms. They also reveal that if there are solvent atoms or molecules occupying the region of space that would be occupied by the excess electron in the absence of the solvent, then the binding energy may become so small that the excess electron would not remain bound if corrections to the $\mathrm{BO}$ approximation were included even though the dipole moment is larger than 2.4 D.

\section{Parallel Tempering Monte Carlo Simulations of the Finite Temperature Behavior of $\left(\mathrm{H}_{2} \mathrm{O}\right)_{6}^{-}$}

\section{General Considerations:}

Electrons in bulk water are of fundamental importance in radiation chemistry, electrochemistry, and biochemistry. ${ }^{88} 92$ The hydrated electron has been known since the early 1960 's. ${ }^{144}$ Anionic water clusters were first observed mass spectroscopically by 
Haberland and coworkers in $1984 .{ }^{116}$ The $\left(\mathrm{H}_{2} \mathrm{O}\right)_{n}{ }^{-}$mass spectrum is dominated by peaks at $n=2,6,7$, and $\geq 11 .{ }^{113,116,145}$ The origin of this intensity pattern is still under debate. Recent advances in vibrational predissociation spectroscopy using Ar solvation techniques have allowed the vibrational spectra of the $n=5$ - 9 anionic water clusters to be determined in the $\mathrm{OH}$ stretch region. ${ }^{58,59}$ The vibrational spectra of the $\left(\mathrm{H}_{2} \mathrm{O}\right)_{n}{ }^{-}$clusters in this size range are similar in appearance, suggesting a common structure motif. Ayotte et al. ${ }^{59}$ have proposed that chain-like structures are responsible for the observed spectra, but alternative models have been proposed. ${ }^{110,146,147}$

Although water clusters do not have low-lying unfilled valence orbitals, an excess electron can bind when the water monomers are arranged so that a sufficiently attractive electrostatic potential results. In those cases that the monomer dipoles are arranged so that the net dipole is in excess of about $2.5 \mathrm{D}$, an excess electron can bind to give a so called dipole-bound anion. ${ }^{8,21,22,35,62,148}$ It was long believed that theoretical methods allowing for the electrostatic interactions, and perhaps also, polarization of the water molecules by the excess electron, were adequate for describing $\left(\mathrm{H}_{2} \mathrm{O}\right)_{n}{ }^{-}$species. $^{21,22,74-}$ ${ }^{77,105}$ Within an ab initio electronic structure framework, this would lead one to believe that the Hartree-Fock method provides a good zeroth-order description of the wavefunction of the anion and of the electron binding energies of these species. This expectation has also served as the basis of several one-electron model approaches that have been developed for describing an excess electron interacting with water. ${ }^{74-77,81}$ These model potentials have been used in Monte-Carlo and molecular dynamics simulations (MD) of excess electrons interacting with bulk water, water films, and water clusters. $^{128-133}$ 
Over the past few years it has been realized that dispersion interactions between an excess electron and the polar molecules in a cluster play a major role in the electron binding. ${ }^{23,24,26,27,32,36,149,150}$ Such interactions are obviously absent in the Hartree-Fock method and in traditional one-electron models. Indeed, it has been found that, within an ab initio framework, $\operatorname{CCSD}(\mathrm{T})^{31}$ or CCSDT calculations ${ }^{32}$ using large, flexible basis sets are required to adequately describe the effects of dispersion in these systems. 23,24,26,27,32,149,150 Ab initio calculations at this level are computationally prohibitive for water clusters containing more than six molecules. Monte-Carlo or MD simulations on $\left(\mathrm{H}_{2} \mathrm{O}\right)_{n}{ }^{-}$clusters based on accurate ab initio energetics (and also forces, in the case of MD simulations) are thus out of the question.

Recently, we introduced a new one-electron model for describing an excess electron interacting with polar molecules. ${ }^{33-35}$ This model, which employs quantum Drude oscillators to describe dispersion interactions, gives electron binding energies comparable to those from ab initio $\operatorname{CCSD}(\mathrm{T})$ calculations, while requiring orders of magnitude less CPU time. In the present work, the Drude model is used to carry out parallel tempering Monte-Carlo simulations ${ }^{151}$ on $\left(\mathrm{H}_{2} \mathrm{O}\right)_{6}{ }^{-}$. Several modifications to the original Drude model code for water which lead to a significant speedup of the program are described.

\section{Drude model}

\section{A. Drude Model for electron-molecule interactions}

The Drude model was originally introduced to describe the dispersion interactions between atoms or molecules. ${ }^{82}$ In the Drude model, two charges, $+q$, and $-q$, coupled 
harmonically through a force constant $k$ are placed on each atom or molecule. The polarizability ( $\alpha$ ) of a Drude oscillator is given by $q^{2} / k$, generally taken to correspond to the experimental polarizability of the atom or molecule of interest. If the Drude oscillators are treated quantum mechanically, a London-type expression is obtained for the dispersion interaction between the oscillators.

In extending this approach to electron-polar molecule interactions, we introduced a coupling term of the form

$$
V^{e D}=\frac{q \mathbf{r} \cdot \mathbf{R}}{r^{3}} f(r),
$$

between the excess electron and a Drude oscillator. ${ }^{33,34}$ Here $\boldsymbol{R}$ is a vector giving the position of the $+q$ charge of the Drude oscillator relative to the $-q$ charge, which is held fixed, $\boldsymbol{r}$ is the position vector of the electron relative to the center of the Drude oscillator, and $f(r)$ is a damping function, chosen to be $1-\exp \left(-b r^{2}\right)$, used to cut off the unphysical short-range interactions. ${ }^{152}$ (In Eq. 42 and in subsequent equations atomic units are employed.) $V^{e D}$ was combined with a model Hamiltonian, $H^{e l}$, allowing for the interaction of the excess electron with the charge distribution of the polar molecules and including pseudopotentials to represent short-range repulsion of the excess electron with the electron distributions of the monomers. The full system was described by the Hamiltonian

$$
H=H^{e l}+H^{o s c}+V^{e D}+V^{D D},
$$

where $H^{o s c}$ and $V^{e D}$ were summed over all the Drude oscillators in the system, and $V^{D D}$ allowed for interactions between the Drude oscillators.

In solving the resulting one-electron Schrödinger equation, a product basis of the form $\left|\phi_{i}(\boldsymbol{r}) \chi_{j}^{(1)}\left(\boldsymbol{R}_{\boldsymbol{I}}\right) \chi_{k}^{(2)}\left(\boldsymbol{R}_{2}\right)^{\cdots}\right\rangle$, where $\phi_{i}$ is a Gaussian type electron orbital and $\chi_{j}^{(m)}\left(\boldsymbol{R}_{\boldsymbol{m}}\right)$ is 
the $j^{\text {th }}$ oscillator level associated with the $m^{\text {th }}$ Drude oscillator, was used. Information on the oscillator and electron basis set are given below. The electron binding energy was obtained by use of a configuration interaction single-plus-doubles (CISD) calculation, where the double excitations allowed and only allowed for simultaneous excitations of the excess electron and of one of the oscillators.

\section{B. Drude Model For Water}

In applying the Drude model to water, the polarizable Dang-Chang (DC) water model, ${ }^{134}$ which has been found to provide a good description of neutral water clusters, ${ }^{134,135,153}$ was employed to represent water-water interactions. ${ }^{34,35}$ The DC model employs a positive charge $(0.519)$ on each $\mathrm{H}$ atom and a negative charge $(-1.038)$ on the rotational axis, but displaced off the $\mathrm{O}$ atom toward the $\mathrm{H}$ atoms. The $\mathrm{DC}$ model also associates a Lennard-Jones site with the $\mathrm{O}$ atom of each monomer and an isotropic polarizable site at the same location as the negative charge. In our modification of the DC model for treating excess electron/water interactions, the polarizable site was replaced with a Drude oscillator with the same polarizability. The Drude oscillators were treated quantum mechanically, for describing both intermolecular induction and for describing the interaction of the excess electron with the oscillators. Intermolecular induction between water molecules was treated by iteratively solving the ground state wavefunction of the Drude oscillators (i.e., $H^{o s c}+V^{D D}$ ) until convergence was reached. This procedure gives net (permanent plus induced) dipoles on each water molecule in the cluster nearly identical to those calculated by solving the classical polarization problem for the original DC model. 
The electronic Hamiltonian including contributions from the induced dipoles has the form:

$$
H_{c m}^{e l}=-\frac{1}{2} \nabla^{2}-\sum_{j} \frac{Q_{j}}{r_{j}}+V^{r e p}+\sum_{i} \frac{\left\langle 0_{i}\left|q \mathbf{R}_{\mathbf{i}} \cdot \mathbf{r}_{i}\right| 0_{i}\right\rangle}{r_{i}^{3}}
$$

where the first sum is over the charge sites of the monomers and the second, which describes the coupling of the excess electron to the induced dipoles, is over the Drude oscillators. The subscript "cm" refers to the choice of atomic charges used in the model. Even though the final Drude model for water employed the DC charges, for the purpose of comparing with ab initio Koopmans' Theorem $(\mathrm{KT})^{67}$ calculations, and for determining the repulsive potential, it was also useful to consider a model employing charges derived from Hartree-Fock calculations on the water monomer. $V^{\text {rep }}$ was derived following the procedure of Schnitker and Rossky. ${ }^{75}$ With this choice of $V^{\text {rep }}$, the excess electron is too weakly bound. To remedy this, the repulsive core was scaled so that for the water dimer the electron binding energy obtained from Eq. (44) when employing Hartree-Fock charges reproduces the ab initio KT result.

\section{Modified Drude Model for Water}

The most CPU-demanding step in a Drude model calculation of electron binding energy is the formation of the CISD matrix and the determination of its lowest eigenvalue. The size of the CISD matrix is of the order $N_{o s c} \cdot n_{o} \cdot n_{e}$, where $N_{o s c}$ is the number of oscillators, $n_{o}$ is the number of harmonic oscillator basis functions associated with each oscillator, and $n_{e}$ is the number of electronic basis functions. Since the electron is weakly bound in a spatially extended orbital, a large set electronic basis functions is required. Based on the strategy for choosing the basis set used in our earlier study of 
$\left(\mathrm{H}_{2} \mathrm{O}\right)_{2}{ }^{-},\left(\mathrm{H}_{2} \mathrm{O}\right)_{3}{ }^{-}$, and $\left(\mathrm{H}_{2} \mathrm{O}\right)_{4}{ }^{-}$, it is anticipated that 80-90 Gaussian basis functions would be required to treat the binding of an excess electron to a cluster the size of $\left(\mathrm{H}_{2} \mathrm{O}\right)_{6}$. Our earlier work employed ten harmonic oscillator basis functions of the form $x^{i} y^{j} z^{k} e^{-\beta\left(x^{2}+y^{2}+z^{2}\right)}$, where $0 \leq i+j+k \leq 2$, for each Drude oscillator. With these basis sets, the dimension of the CI matrix for $\left(\mathrm{H}_{2} \mathrm{O}\right)_{6}{ }^{-}$would be on the order of 5000. MonteCarlo simulations requiring construction of a matrix of this size and extraction of its lowest eigenvalue at each step would be computationally prohibitive, particularly if the simulations were to be run long enough to ensure convergence.

Significant reduction in CPU time would result if it were possible to employ only four, i.e. $s, p_{x}, p_{y}, p_{z}$-like basis functions per oscillator. However, test calculations reveal that large errors result in the calculated dispersion energies if this smaller basis set is used for the oscillators. This is on account of the dual role of the oscillators in describing intramolecular induction as well as electron-molecule dispersion. In essence, the induction interactions cause a mixing between the $s$ and $p$ oscillator functions which, in turn, makes excitations into $d$-like oscillator functions important for describing electronwater dispersion interctions. This analysis suggests that the errors due to the use of the four-function oscillator basis set could be significantly reduced were the intramolecular polarization described classically and the quantum Drude oscillators used to describe the electron-oscillator interactions only. In this approach, the electronic Hamiltonian becomes

$$
H_{c m}^{e l^{\prime}}=-\frac{1}{2} \nabla^{2}-\sum_{j} \frac{Q_{j}}{r_{j}}+\sum_{i} \frac{\mu_{i} \cdot r_{i}}{r_{i}^{3}}+V^{r e p},
$$


where $\mu_{j}$ is the induced dipole moment on the $j^{\text {th }}$ water molecule calculated by solving the classical self-consistent polarization equations.

Test calculations on various $\left(\mathrm{H}_{2} \mathrm{O}\right)_{6}{ }^{-}$isomers reveal that the modified Drude model with the smaller oscillator basis set gives nearly the same electron binding energies as the original approach (Eq. 44) in which both intramolecular induction and electron-molecule dispersion were treated quantum mechanically using Drude oscillators and ten basis functions were employed per oscillator. The adoption of the Hamiltonian described in Eq. 45 and the smaller four-function oscillator basis set results in over an order of magnitude reduction in the CPU time. Further performance improvement resulted from implementing a semi-direct CI method to obtain the lowest eigenvalue of the CI matrix. With these changes, the time to form the CISD matrix and to determine its lowest eigenvalue for a cluster of the size $\left(\mathrm{H}_{2} \mathrm{O}\right)_{6}{ }^{-}$is reduced to about $1 \mathrm{sec}$ on a $2 \mathrm{Ghz}$ Pentium IV computer. However, the total CPU time, including evaluating the integrals over the repulsive cores, is about 10 seconds.

As will be discussed below, a multi-center expansion of Gaussians was used as the basis set for the excess electron. To facilitate integral evaluation, the repulsive core was also represented in terms of Gaussian functions. The CPU timings cited above were obtained using the 59-Gaussian function representation of the repulsive core on each water monomer developed in Ref. 34. Due to the complicated representation of the repulsive core, a large number of three-center integrals involving the repulsive core and the electronic basis functions result, making this the most time consuming part of the calculation after the changes described above have been implemented to speed up the formation of the CISD matrix and the determination of the lowest eigenvalue. To address 
this problem, a new repulsive core with only three $s$ Gaussians on each Hydrogen atom and no functions on the Oxygen atom was developed. As before, this new repulsive core was rescaled so that the electron-binding energy for $\left(\mathrm{H}_{2} \mathrm{O}\right)_{2}{ }^{-}$obtained using Eq. 45 with Hartree-Fock charges reproduced that from the ab initio KT-level calculations. The electron binding energies for larger $\left(\mathrm{H}_{2} \mathrm{O}\right)_{n}{ }^{-}$clusters obtained using this new repulsive core are nearly identical to those obtained using the 59-function representation of the repulsive core. All results discussed below were obtained using the new repulsive core. With the modified Drude model and the simplified repulsive core the total CPU time for an energy evaluation for $\left(\mathrm{H}_{2} \mathrm{O}\right)_{6}{ }^{-}$is about $2 \mathrm{sec}$ on a $2 \mathrm{GHz}$ Pentium IV PC.

\section{Basis set and Parameters}

In describing the binding of excess electrons to water clusters, large, flexible basis sets must be employed. When using localized basis functions, as is done in this work, there rises the problem of where these functions should be centered. This is particularly problematical in Monte-Carlo (or molecular dynamics) simulations exploring a wide range of geometrical configurations. For example, in our T=190 K Monte-Carlo simulations of $\left(\mathrm{H}_{2} \mathrm{O}\right)_{6}{ }^{-}$, the dipole moment of the neutral cluster varies from almost zero to about 10 Debye, and, thus, the basis set must be flexible enough to allow for a wide range of spatial distributions of the excess electron. At the same time, the number of basis functions has to be kept as small as possible to minimize computational cost. One approach would be to locate a large set of $s$ and $p$ Gaussian functions on each monomer. Such an approach would be both very demanding computationally and prone to linear dependency. The alternative of putting a large set of Gaussian functions on a single center would require inclusion of very high angular momentum functions to converge the 
electron binding energy for an arbitrary geometry and thus would also be computationally demanding. A compromise, which works well for many systems, is to combine a large single-center expansion of diffuse $s$ and $p$ functions, perhaps floating, with smaller sets of $s$ and $p$ functions centered on each monomer. In our initial application of the Drude model to water clusters, the large single-center expansion of $s$ and $p$ functions was augmented with a $2 s 2 p$ set of Gaussian functions on each Hydrogen atom.

In the present study, the $2 s 2 p$ basis set on each $\mathrm{H}$ atom was replaced by a $2 s 1 p$ basis set, thereby reducing the overall CPU time for a Drude model calculation on $\left(\mathrm{H}_{2} \mathrm{O}\right)_{6}{ }^{-}$by about a factor of two. The exponents of the two $s$ Gaussian functions, 0.1027 , and 0.02526 , were taken from our earlier work, as was the exponent of the $p$ function (0.141). In the latter case, the tighter $p$ function was retained. In order to compensate for the small $(\leqslant 5 \%)$ decrease in electron binding energies due to the removal of the second $p$ function on each $\mathrm{H}$ atom, the repulsive scaling factor and the damping parameter $b$ were adjusted slightly so that the electron binding energies of $\left(\mathrm{H}_{2} \mathrm{O}\right)_{2}{ }^{-}$calculated using the Drude model, with and without inclusion of dispersion interactions, reproduced the corresponding values obtained with the original Drude model, using the larger $2 s 2 p$ basis set on each $\mathrm{H}$ atom. (The same large single-center electron basis set was used in both cases.) This gave a scaling factor of 7.0 and a damping parameter of 0.5 .

For the Monte-Carlo simulations described below, the large single-center set of diffuse functions was chosen to consist of an even-tempered sequence of five $s$ functions with exponents ranging from $7.5^{*} 10^{-2}$ to $1.2^{*} * 10^{-4}$, and an even-tempered sequence of four $p$ functions with exponents ranging from $6.0^{*} 10^{-2}$ to $4.8^{*} 10^{-4}$, both located at the center of mass of the cluster. Table X compares the electron binding energies for five isomers of 
$\left(\mathrm{H}_{2} \mathrm{O}\right)_{6}{ }^{-}$as calculated using the modified Drude model both with the $5 s 4 p$ single-center expansion and with a larger $8 s 7 p$ single-center expansion, the location of which was variationally optimized. (In both cases, the electronic basis set also included the two $s$ and one $p$ function on each $\mathrm{H}$-atom as described above.) Excellent agreement is found between the electron binding energies calculated using the Drude model with the larger $8 s 7 p$ basis set and those obtained from ab initio calculations. The Drude model calculations reveal that the dispersion and higher order contributions to the electron binding energies are exceedingly important, ranging from 164 to $391 \mathrm{meV}$, depending on the isomer. It is also found that the Drude model employing the smaller single-center expansion, located at the center of mass, underestimates the electron binding energies. However, with the exception of the chain isomer, the errors in the electron binding energies due to the restrictions on the electronic basis set are quite small $(<10 \%)$. For the chain isomer, the error is $19 \%(70 \mathrm{meV})$, reflecting the inadequacy of the relatively small $(5 s 4 p)$ single-center basis set, when located so far $(\approx 7 \AA)$ from the terminal acceptor monomer. Nonetheless, the smaller $5 s 4 p$ single-center expansion should suffice for the purpose of the Monte-Carlo simulations, which are intended to provide information on the relative populations of different isomers as a function of temperature.

\section{Parallel Tempering Simulations}

The Monte-Carlo simulations were carried out using the parallel tempering algorithm ${ }^{151}$ to avoid quasi-ergodic behavior caused by large energy barriers. With this algorithm, simulations for a series of replicas (each at a different temperature) are carried out in parallel, with most attempted moves being confined to individual replicas and carried out using the Metropolis algorithm. The remaining attempted moves involve 
exchanges of configurations from replicas at adjacent temperatures. The acceptance criterion used for these moves assures detailed balance is obeyed. ${ }^{151}$

In this study, simulations of $\left(\mathrm{H}_{2} \mathrm{O}\right)_{6}$ and $\left(\mathrm{H}_{2} \mathrm{O}\right)_{6}{ }^{-}$were carried out at eight temperatures, in a geometric ratio ${ }^{154}$ from 50 to $190 \mathrm{~K}$. The highest temperature was chosen so that potential energy barriers are readily overcome. Exchanges between replicas were attempted every 60 trial moves. For the simulations on the neutral clusters, an acceptance ratio of 40-50\% was observed for exchanges between each pair of "adjacent" replicas. For the simulations on the anionic clusters, an acceptance ratio of about $30-40 \%$ was achieved for the exchanges with the exception of those between the 157 and $190 \mathrm{~K}$ replicas, for which acceptance ratio was only about $10 \%$. This is problematical only in that it makes the simulation less efficient, and more steps are required to reach ergodicity.

The water monomers were kept rigid at the DC geometry. Each individual trial move involved either a translation or a rotation of one or more water molecules. Both the number of water monomers and the specific water monomers in the moving unit were chosen at random. Test calculations show that larger step sizes can be used if two or more water molecules are sometimes moved as a subunit instead of individually as is usually done in Monte-Carlo simulations. The maximum translational and rotational steps were adjusted dynamically and independently every 1000 trial moves to maintain an acceptance ratio of 0.5 for the Metropolis algorithm. No evaporative events were found at any of the temperatures employed.

In simulating the anionic water clusters, there is the distinct possibility of sampling configurations for which the "anion" lies energetically above the neutral cluster 
(at the same geometry). Such configurations would be subject to electron autoionization, giving the neutral cluster plus a free electron. In our simulations the electron cannot escape to an infinite distance from the cluster due to the constraints of the basis set. This, of course, could make recapture processes more prevalent than in the experiments.

The initial geometries for the simulations were chosen at random. The simulations were pre-equilibrated for 200,000 steps, after which the average potential energy and heat capacity at each temperature were no longer rapidly changing. The production simulations were carried out for 2,000,000 steps for each temperature, with 3997 structures, chosen at a fixed interval, being saved for each replica.

In the simulations of the anion, the dipole moment of the corresponding neutral cluster was monitored, and the electron binding energy was recalculated only if the dipole moment change associated with an individual move or with a sequence of consecutive moves exceeded $2 \%$ in magnitude. For dipole moment changes smaller than this, the total energy was calculated by adding to the energy of the neutral cluster at the present geometry the electron binding energy from the previous iteration. This strategy was motivated by the observation, that, in general, for moves for which the dipole moment is not appreciably altered, the changes in the total energy tend to be dominated by the energy changes associated with the neutral cluster. In order to deal with the possibility that two completely different structures with similar dipole moments are connected by a pathway of very similar dipole moment intermediate configurations, the electron binding energies were always recalculated after ten consecutive trial moves even if the dipole moment did not change by more than $2 \%$. This strategy led to a factor of two savings in CPU time for the high temperature simulations and a factor of five savings for the low 
temperature simulations. This was taken into account in distributing the various replicas over CPUs, so that, more low-temperature replicas were run on a given CPU than were high temperature replicas. For the 3997 saved geometries, the electron binding energies were recalculated and compared with those estimated using the procedure described above. These calculations show that the errors in the electron binding energies and total energies due to reuse of the electron binding energies from previous geometries never exceeded $5 \%$ and $2 \%$, respectively.

\section{Results and Discussion}

Of particular interest are the populations of various structures as a function of temperature. We first attempted to characterize the structures by determining the inherent structures, ${ }^{155}$ i.e., by optimizing sampled structures to the "closest" local minima. However, this approach was found to greatly undercount chain-like structures for the anionic clusters since most such structures collapsed to non-chain structures during the conjugate-gradient-like minimization. To address this problem, we employed instead the moment of inertia to distinguish structures. The moment of inertia tensor of the cluster

was calculated using $J_{s_{i}, s_{j}}=\sum m \cdot s_{i} \cdot s_{j}$, where $m$ is the mass of the water monomer, and $s_{i}$ and $s_{j}$ are Cartesian coordinates of the center of mass of a specific monomer, with the origin taken to be the center of the mass of the entire cluster. The principle axes were chosen so that the off-diagonal elements of the inertia tensor were zero. The three principal moments of inertia satisfy $J_{x} \leq J_{y} \leq J_{z}$. The motivation for this approach is the realization that chain-like structures should have larger $J_{z}$ values than non-chain structures. Histograms giving the population as a function of $J_{z}$ are plotted and analyzed. 
Roughly speaking, the $J_{z}$ values can be mapped onto structures as reported in Table XI. This Table includes only a subset of possible isomers, in particular, those which acquire significant population in one or more replicas as well as the ring structure. Several configurations sampled in the simulations at $\mathrm{T}=157$, and $190 \mathrm{~K}$ together with their $J_{z}$ values are shown in Figure 14. This figure and Table XI associated names such as "cage", "prism" and "open-book" to various configurations. However it should be noted that the cage, prism, and open-book structures for the anionic cluster have different arrangements of the H-bonds in the neutral clusters.

Figure 15a reports for neutral cluster the $J_{z}$ histograms for a subset of the eight replicas. The area under the various curves in Figure 15a were integrated with respect to $J_{z}$ and the cumulative populations are plotted in Figure 15b. In the lowest temperature replica $(50.0 \mathrm{~K})$, there are pronounced peaks near $J_{z}=6.25$ and 7.6, corresponding to prism-like, and cage-like structures, respectively. At a temperature of $60.5 \mathrm{~K}$, nearly all the population is associated with prism-like structures. At $\mathrm{T}=88.6 \mathrm{~K}$ the population of prism structures has fallen off somewhat and a small population of broken prism (with one Hydrogen-bond of the prism broken and $\left.J_{z} \approx 7.0\right)$ and distorted cage $\left(J_{z} \approx 8.5\right)$ structures has built up. As the temperature is further increased, the population shifts mainly to open-book and ring structures $\left(J_{z}=9-10\right)$. These results are consistent with previous Monte-Carlo simulations of $\left(\mathrm{H}_{2} \mathrm{O}\right)_{6} \cdot{ }^{135}$. Chain-like structures are not detected for the neutral cluster at temperatures as high as $190 \mathrm{~K}$.

Figure 16a reports for $\left(\mathrm{H}_{2} \mathrm{O}\right)_{6}{ }^{-}$the $J_{z}$ histograms for a subset of the eight replicas. The cumulative populations are shown in Fig. 16c. At the lowest temperature (50 K) considered, most $(\approx 95 \%)$ of the population is associated to prism-like structures with $J_{z}$ 
values between 6 and 7. At $60.5 \mathrm{~K}$, about $80 \%$ of the population remains associated with prism-like structures and with most of the remaining population being due to cage-like structures $\left(J_{z} \approx 8.0\right)$. Inspection of the configurations sampled at 157 and $190 \mathrm{~K}$ reveals that open-book (Fig. 14c), cage-plus-tail (Fig. 14d), ring-plus-tail (Fig. 14e, 14f), ringplus-two-tails (Fig. 14g) and chain-like structures (Fig. 14h, 14i) are abundant. Although the chain-like structures account for only about $2 \%$ of the structures at $157 \mathrm{~K}$, at $190 \mathrm{~K}$ they account for about $50 \%$ of the observed configurations. Since the basis set used for the simulations underestimate by about $70 \mathrm{meV}$ the stability of the chain-like isomer (at its optimized geometry), it is expected that chain-like structures would be somewhat more important at the lower temperatures were a more flexible basis set adopted.

These results show that for the $\left(\mathrm{H}_{2} \mathrm{O}\right)_{6}$ " there is an evolution from "compact" clusters to more "open” structures with increasing temperature. The evolution corresponds to the breaking of Hydrogen-bonds, with the maximum number (9) of the Hydrogen-bonds being for the prism-like structures that dominates at low temperature and the smallest number (5) of Hydrogen-bonds being for the chain-like structures that dominate at high temperatures.

Both the tweezers structure depicted in Table $X$ and Figure $17 \mathrm{e}^{146,156}$ and the dbs4'dbs 2 structure depicted in Table $X$ and Figure $17 \mathrm{~d}^{157}$ may be the dominant species observed experimentally. A common feature of these three structures is the fourmembered-ring at the "bottom" of the isomer and the two water molecules on the "top" of the four-membered-ring. In the tweezers structure, the two "on top" molecules are not bonded to one another and have their dipoles oriented so that the excess electron can be partially localized between them. In the dbs4'dbs2 structure the two "on-top" monomers 
are bonded together, and the single-donor $\mathrm{OH}$ groups of the four-membered-ring are oriented counter-clockwise when viewed from the "top" of the cluster. It is clear that there should be another isomer, denoted here as dbs4dbs2, with a clockwise orientation of the single-donor $\mathrm{OH}$ groups of the four-membered-ring. To the best of our knowledge, the dbs4dbs2 isomer has not been previously described in the literature. Our Drude model calculations predict that at their optimized structures the dbs $4 \mathrm{dbs} 2$ is about $1.2 \mathrm{kcal} / \mathrm{mol}$ more stable than the dbs4'dbs2 isomer.

The tweezers structures have $J_{z}$ values close to those of the cage structures, whereas the dbs4dbs2 and dbs4'dbs2 isomers have $J_{z}$ values close to that of the prism isomers. Thus, the $J_{z}$ values alone do not enable us to determine whether these isomers are present in the simulations. To address this issue, for each replica, we identified from the saved configurations all those with $J_{x}, J_{y}$, and $J_{z}$ values, dipole moments, and total energies within specified tolerances (10\% to $20 \%$ ), of the corresponding values associated with the dbs4dbs2, dbs4'dbs2, and tweezers isomers at their optimized geometries. The structures thus selected were visually inspected to determine their structures. Table XII reports the number of times that each of these three structures was observed out of 3997 sampled configurations at each of the eight temperatures considered.

The tweezers structure was not detected in the sampled configurations from any of the replicas. Both the dbs4dbs2 and dbs4'dbs2 structures were detected, with one to three examples of the latter being detected in the $\mathrm{T}=88.6-190 \mathrm{~K}$ replicas. The more stable dbs4dbs2 species was sampled in each of the $\mathrm{T}=60.5-190 \mathrm{~K}$ replicas, occurring most frequently (20 times or $0.5 \%$ of the population) in the $157 \mathrm{~K}$ replica. Thus, none of 
the dbs4dbs2, dbs4'dbs2, or tweezers structures acquire sizable population under equilibrium conditions. Although the experimental formation process may result in a nonequilibrium distribution, it is likely that none of these isomers is responsible for the dominant $\left(\mathrm{H}_{2} \mathrm{O}\right)_{6}{ }^{-}$ion observed experimentally.

\section{Conclusions}

Our Monte-Carlo simulations of the anionic $\left(\mathrm{H}_{2} \mathrm{O}\right)_{6}{ }^{-}$cluster indicate that chainlike structures account for about $50 \%$ of the configurations sampled at a $\mathrm{T}=190 \mathrm{~K}$ simulation. Although, early in the expansion neutral water clusters could have temperatures near this value, our simulations of the neutral cluster do not reveal any chain-like structures even at $\mathrm{T}=190 \mathrm{~K}$. Rather, open-book and ring-like structures dominate for the neutral cluster at this temperature. Thus formation of $\left(\mathrm{H}_{2} \mathrm{O}\right)_{6}{ }^{-}$clusters with chain-like structures via electron capture by neutral clusters with an isomer distribution reflecting that present in a $190 \mathrm{~K}$ equilibrium distribution, would require breaking one or two Hygrogen-bonds. In clusters with Ar atoms attached, the cluster temperature should be below $50 \mathrm{~K}$, which implies that were equilibrium attained, prismlike anionic clusters should dominate.

The present study predicts the prism (Fig. 17a), cage (Fig. 17b), and dbs4dbs2 (Fig. 17h) isomers of $\left(\mathrm{H}_{2} \mathrm{O}\right)_{2}{ }^{-}$to be particularly stable with total binding energies being $48.90,-48.32$, and $-48.16 \mathrm{kcal} / \mathrm{mol}$, respectively. The corresponding vertical electron detachment energies are $376 \mathrm{meV}, 437 \mathrm{meV}, 658 \mathrm{meV}$, as compared to the $480 \mathrm{meV}$

experimental value. ${ }^{58}$ The Drude model calculations used rigid water monomers, and, based on test calculations on $\left(\mathrm{H}_{2} \mathrm{O}\right)_{3}{ }^{-}$and $\left(\mathrm{H}_{2} \mathrm{O}\right)_{4}{ }^{-}$, the calculated VDE's would be expected to increase by $30-50 \mathrm{meV}$ were this constraint relaxed. Hence both the prism 
and cage structures of $\left(\mathrm{H}_{2} \mathrm{O}\right)_{6}{ }^{-}$have electron binding energies fairly close to the $480 \mathrm{meV}$ experimental value. Additional experimental and theoretical work will be required to establish whether either of these two isomers is indeed responsible for the dominant form of $\left(\mathrm{H}_{2} \mathrm{O}\right)_{6}{ }^{-}$observed experimentally.

\section{Use of the designation "dipole-bound"}

In recent years there has been a tendency to reserve the term "dipole-bound" anion for species in which the electron binding energy is relatively weak, e.g., less than $0.1 \mathrm{eV} .{ }^{158,159}$ According to such a criterion, the ground state anions of the chain form of $\left(\mathrm{H}_{2} \mathrm{O}\right)_{6}$ and of $\mathrm{NaCl}$, for example, would not be classified as dipole-bound. Although the excess electron is bound by over $0.4 \mathrm{eV}$ in these species, ${ }^{113,145,160}$ most of its charge density is still "outside" of the valence region of the neutral molecule or cluster. Also the one-electron Drude-type model is as successful at describing binding of an excess electron to $\left(\mathrm{H}_{2} \mathrm{O}\right)_{6}$ and to $\mathrm{NaCl}$ as to species such as $\mathrm{CH}_{3} \mathrm{CN}$ and $\left(\mathrm{H}_{2} \mathrm{O}\right)_{2}$, which display much weaker electron binding. For these reasons, we believe that it is also appropriate to refer to these more strongly bound anions as dipole-bound.

It is interesting to consider the application of the one-electron Drude model to ionic species, using $\mathrm{NaCl}$ as the example. There are two ways of setting up a Drude model for describing an excess electron interacting with $\mathrm{NaCl}$ : (1) choosing atomic charges that reproduce the experimental dipole moment and employing a single polarizable site with the polarizability chosen to reproduce the experimental molecular polarizability, or (2) employing charges of +1 and -1 on the $\mathrm{Na}$, and $\mathrm{Cl}$, respectively, and introducing Drude sites on each ion and allowing polarization of each Drude oscillator by the other ion. (Since $\mathrm{Na}^{+}$is much less polarizable than $\mathrm{Cl}^{-}$, to a good approximation, only 
a single Drude oscillator located on the $\mathrm{Cl}^{-}$ion, need be employed.) In the second approach, it is necessary to damp the interactions of the ions with the Drude oscillators to obtain a correct value of the induced dipole moment. For ionic species such as $\mathrm{NaCl}$, in contrast to the non-ionic species discussed above, the polarization and dispersion contributions to the electron binding energy are comparable in magnitude and are small compared to the electrostatic contribution to the binding of the excess electron. This is a consequence of the more highly-localized excess electron in $\mathrm{NaCl}$ compared to the more weakly-bound anions discussed above.

\section{8. "Solvated-electron" and related systems}

Arrangements of polar molecules with small, or even zero, net dipole moments can bind excess electrons. ${ }^{43,161,162}$ Geometries with the positive ends of the monomer dipoles pointing toward one another and with the excess electron "trapped" in the resulting attractive potential in the interior of the cluster are often referred to as "solvated-electron" species. The hydrated electron ${ }^{103,105,107}$ is probably the most famous solvated-electron species. (Here we have assumed that the commonly accepted picture of the hydrated electron - in which the electron is bound in an approximately spherical cavity surrounded by water molecules with "free" $\mathrm{OH}$ groups pointing toward the center of the cavity - is correct. Recent theoretical studies have demonstrated that dimers of various biomolecules such as urea can also form solvated-electron anions. ${ }^{163}$

To illustrate the properties of solvated-electron species, we consider the $(\mathrm{HF})_{n}{ }^{-}, n$ $=2-6$ clusters, with the molecules arranged so that the net dipoles are zero. These species have been examined in detail by Gutowski and co-workers, ${ }^{70,161}$ and the geometrical arrangements of the first three members of this species are shown in Figure 18. Although 
these structures are obviously unstable for the neutral molecules, the resulting anions are stable in the sense that, they lie energetically below the neutral clusters at the same geometry arrangement. There are other geometrical arrangements of the $(\mathrm{HF})_{n}{ }^{-}$clusters that are intermediate between dipole-bound and symmetric solvated-electron in nature. The asymmetrically solvated species are typically more stable than the fully-symmetric solvated species. ${ }^{161}$ There is experimental evidence for an asymmetrically solvated form of $(\mathrm{HF})_{3}-161$

Table XIII summarizes the contributions, obtained from ab initio calculations, of various terms to the electron binding energies for $(\mathrm{HF})_{n}{ }^{-}, n=2-6$, solvated-electron species. In each case, the results are reported at the geometry for which the anion, constrained to the symmetry indicated in the Table and described by MP2 calculations, has its potential energy minimum. From comparison of the results in this Table with those in Table I, it is seen that the dispersion contributions to the electron binding energies are even more important for the "solvated-electron" systems than for the corresponding dipole-bound anions. For example for $(\mathrm{HF})_{3}{ }_{3}$, the second-order dispersion contribution to the electron binding energy is $427 \mathrm{meV}$ for the solvated-electron species but only $77 \mathrm{meV}$ for the corresponding dipole-bound anion. In part, this is a consequence of the strong dependence of the dispersion interaction between the excess electron and a particular monomer on the distance of the monomer from the excess electron. (See Eq. 25.) In chain-like isomers, the dispersion contribution to the electron binding is greatest for the terminal monomer closest to the excess electron, and falls off rapidly, along the chain. 
Skurski et al ${ }^{164,165}$ have also considered species with two dipoles oriented as shown in Figure 19a which also have zero net dipole moment. These authors have referred to electrons bound to this charge arrangement as "bi-dipole" bound. Molecules or clusters with charge arrangements such as those shown in Figures 19b and 19c, also have zero net dipole moments and may bind an excess electron to give a so-called quadrupole-bound anions. ${ }^{62,148,166-169}$ The ground state of $(\mathrm{BeO})_{2}$ anion with a $\mathrm{D}_{2 \mathrm{~h}}$ geometry is an example of a quadrupole-bound anion. ${ }^{167,168}$ Although one can refer to $(\mathrm{BeO})_{2}{ }^{-}$as quadrupole bound, such species do not have a critical quadrupole moment analogous to the critical dipole moment for electron binding in dipole fields. ${ }^{148,166,169} \mathrm{It}$ has been proposed that formamide dimer, p-dinitrobenzene, and even $\mathrm{CS}_{2}$ form quadrupole-bound anions. ${ }^{39,170-172}$ However, we are unaware of any conclusive ab initio calculations that demonstrate quadrupole bound anions for these species.

The solvated-electron, bi-dipole-bound and quadrupole-bound anion species are closely related in that, the binding of an excess electron in each case (as well as in dipolebound anions) results from the existence of a highly attractive electrostatic potential over a sufficiently large region of space. If the highly attractive region does not "span" a large region of space, then by consequence of the uncertainty principle, "confinement" of the electron is accompanied by a large increase in the kinetic energy causing it to become unbound. For this reason, the solvated-electron form of $(\mathrm{HF})_{2}{ }^{-}$becomes unstable to electron detachment when the $\mathrm{H}$ atoms of the two HF molecules are brought to a separation of about $5.2 \AA$. 


\section{Conclusions}

In this thesis we have focused on recent theoretical work on dipole-bound anions and the closely related solvated-electron species. Particular emphasis has been placed on elucidating the role of electron correlation effects, especially dispersion-type interactions in describing the binding of an excess electron. Dispersion-type interactions make large contributions to the electron binding in both dipole-bound and solvated-electron anions. This is an important observation since, until very recently, all one-electron models introduced to treat excess electrons interacting with water and other polar molecules, have neglected dispersion-type interactions. It is demonstrated, that these interactions can be quantitatively described within a one-electron framework by the use of quantum Drude oscillators. The Drude model approach results in a huge reduction of computer time compared to traditional ab initio electronic structure methods. For example, for $\left(\mathrm{H}_{2} \mathrm{O}\right)_{12}{ }^{-}$, a single-point Drude model calculation using the CI method requires only several seconds of CPU time on a Pentium IV PC, whereas an ab initio CCSD(T) calculation on this anion, described by a flexible basis set, would take several weeks of CPU time.

This made possible parallel tempering Monte-Carlo simulations on anionic water clusters. Simulations on $\left(\mathrm{H}_{2} \mathrm{O}\right)_{6}{ }^{-}$carried out at eight different temperatures revealed three new forms of lowest energy isomers. This paved the road to further calculations that may lead to final elucidation of the experimental IR vibrational spectra.

The importance of dispersion effects in the binding of excess electrons as dipolebound or solvated-electron anions is sometimes "masked" in ab initio treatments since charge renormalization effects often act so as to make the electrostatic potential less 
attractive for the excess electron and thus to decrease electron binding. Higher-order electron correlation effects can also significantly impact electron binding energies and can cause a significant contraction of the charge distribution of the excess electron, which has important implications for vibrational spectroscopy of dipole-bound and solvatedelectron species.

The need to include high-order correlation effects, e.g., by means of the $\operatorname{CCSD}(\mathrm{T})$ procedure, greatly restricts the size system for which accurate vibrational spectra can be calculated which is, for example, a significant handicap in making definitive assignments of the isomers responsible for the observed $\left(\mathrm{H}_{2} \mathrm{O}\right)_{6}{ }^{-}$IR spectra. The new generation of model potentials employing Drude oscillators has the potential of solving this problem, but first it will be necessary to remove the constraint of rigid water monomers and to derive expressions for analytical gradients. The computational speed of the one-electron Drude model approach should permit coupling with approaches such as the vibrational SCF method ${ }^{208}$, which would allow for inclusion of anharmonicity effects in calculation of the spectra. 


\section{Reference:}

1 Part of this thesis is reproduced from Ref. 33 Ref. 34, Ref. 35, and Ref.37

E. Fermi and E. Teller, Phys. Rev. 72, 399 (1947).

R. F. Wallis, R. Hermans, and H. W. Milnes, J. Mol Spectrosc 4, 51 (1960).

O. H. Crawford, Proc. R. Soc. London 91, 279 (1967).

O. H. Crawford and A. Dalgarno, Chem. Phys. Lett. 1, 23 (1967).

W. B. Brown and R. E. Roberts, J. Chem. Phys. 46, 2006 (1967).

J. E. Turner, V. E. Anderson, and K. Fox, Phys. Rev. 174, 81 (1968).

W. R. Garrett, Phys. Rev. A. 3, 961 (1971).

W. R. Garrett, J. Chem. Phys. 73, 5721 (1980).

W. R. Garrett, J. Chem. Phys. 77, 3666 (1982).

K. M. Griffing, J. Kenney, J. Simons, and K. D. Jordan, J. Chem. Phys. 63, 4073 (1975).

K. D. Jordan and W. Luken, J. Chem. Phys. 64, 2760 (1976).

K. D. Jordan and J. Wendoloski, Chem. Phys. 21, 145 (1977).

K. D. Jordan and J. Wendoloski, Mol. Phys. 35, 223 (1978).

G. H. Shortley, Phys. Rev. 38, 120 (1931).

L. D. Laudau and E. M. Lifshitz, in Quantum Mechanics (Pergamon Press, Oxford, 1959), pp. 118.

M. Born and R. Oppenheimer, Ann. Phys. 84, 457 (1927).

O. H. Crawford, Chem. Phys. Lett. 2, 461 (1968).

W. R. Garrett, Chem. Phys. Lett. 5, 393 (1970).

D. C. Clary, J. Phys. Chem. 92, 3173 (1988).

K. D. Jordan, Acc. Chem. Res. 12, 36 (1979).

J. Simons and K. D. Jordan, Chem. Rev. 87, 535 (1987).

M. Gutowski, P. Skurski, A. I. Boldyrev, J. Simons, and K. D. Jordan, Phys. Rev.

A. 54, 1906 (1996).

M. Gutowski, K. D. Jordan, and P. Skurski, J. Phys. Chem. A 102, 2624 (1998).

K. Yokoyama, G. W. Leach, J. B. Kim, W. C. Lineberger, A. I. Boldyrev, and M. Gutowski, J. Chem. Phys. 105, 10706 (1996).

M. Gutowski and P. Skurski, Recent Res. Dev. Phys. Chem. 3, 245 (1999).

M. Gutowski and P. Skurski, Chem. Phys. Lett. 300, 331 (1999).

L. Adamowicz, J. Chem. Phys. 91 (12), 7787 (1989).

L. Adamowicz and E. A. McCullough, J. Phys. Chem. 88, 2045 (1984).

C. Desfrancois, V. Periquet, S. Carles, J. P. Schermann, D. M. A. Smith, and L. Adamowicz, J. Chem. Phys. 110 (9), 4309 (1999).

J. A. Pople, M. Head-Gordon, and K. Raghavachari, J. Chem. Phys. 87, 5968 (1987).

K. A. Peterson and M. Gutowski, J. Chem. Phys. 116, 3297 (2002).

F. Wang and K. D. Jordan, J. Chem. Phys. 114 (24), 10717 (2001).

F. Wang and K. D. Jordan, J. Chem. Phys. 116 (16), 6973 (2002).

K. D. Jordan and F. Wang, Ann. Rev. Chem. Phys. 54, 367 (2003).

M. Sindelka, V. Spirko, and P. Jungwirth, J. Chem. Phys. 117, 5113 (2002).

F. Wang and K. D. Jordan, J. Chem. Phys. (submitted). 
R. N. Compton, H. S. Carman, Jr., C. Desfrancois, H. Abdoul-Carime, J. P. Schermann, J. H. Hendricks, S. A. Lyapustina, and K. H. Bowen, J. Chem. Phys. 105, 3472 (1996).

C. Desfrancois, V. Periquet, S. A. Lyapustina, T. P. Lippa, D. W. Robinson, and K. H. Bowen, J. Chem. Phys. 111, 4569 (1999).

F. Lecomte, S. Carles, C. Desfrancois, and M. A. Johnson, J. Chem. Phys. 113 (24), 10973 (2000).

D. M. A. Smith, J. Smets, and L. Adamowicz, J. Phys. Chem. A 103 (21), 4309 (1999).

D. M. A. Smith, J. Smets, and L. Adamowicz, J. Phys. Chem. A 103 (29), 5784 (1999).

C. S. Hall and L. Adamowicz, J. Phys. Chem. A 106 (25), 6099 (2002).

A. F. Jalbout, J. Smets, and L. Adamowicz, Chem. Phys. 273 (1), 51 (2001). J. H. Hendricks, S. A. Lyapustina, H. L. de Clercq, J. T. Snodgrass, and K. H. Bowen, J. Chem. Phys. 104, 7788 (1996).

P. Skurski and J. Simons, J. Chem. Phys. 115 (18), 8373 (2001).

C. Desfrancois, S. Carles, and J. P. Schermann, Chem. Rev. 100, 3943 (2000).

J. Rak, P. Skurski, and M. Gutowski, J. Chem. Phys. 114, 10673 (2001).

M. Gutowski, P. Skurski, and J. Simons, J. Am. Chem. Soc. 122, 10159 (2000).

P. Skurski, M. Gutowski, R. Barrios, and J. Simons, Chem. Phys. Lett. 337, 143 (2001).

A. V. Davis, M. T. Zanni, C. Frischkorn, and D. M. Neumark, J. Electron

Spectrosc (108), 203 (2000).

D. Majumdar, J. Kim, and K. S. Kim, J. Chem. Phys. 112 (1), 101 (2000).

D. Serxner, C. E. Sessent, and M. A. Johnson, J. Chem. Phys. 105, 7231 (1996).

F. Vila and K. D. Jordan, J. Phys. Chem. A 106, 1391 (2002).

H.-Y. Chen and W.-S. Sheu, J. Am. Chem. Soc. 122, 7534 (2000).

H.-Y. Chen and W.-S. Sheu, Chem. Phys. Lett. 335, 475 (2001).

F. Guthe, M. Tulej, T. Mickhail, V. Pachkov, and J. P. Maier, Astrophys. J. 555, 466 (2001).

P. Ayotte, C. G. Bailey, J. Kim, and M. A. Johnson, J. Chem. Phys. 108, 444 (1998).

P. Ayotte, G. H. Weddle, C. G. Bailey, M. A. Johnson, F. Vila, and K. D. Jordan, J. Chem. Phys 110 (13), 6268 (1999).

L. Lehr, M. T. Zanni, C. Frischkorn, R. Weinkauf, and D. M. Neumark, Science 284, 635 (1999).

C. Desfrancois, H. Abdoul-Carime, N. Khelifa, and J. P. Schermann, Phys. Rev. Lett. 73 (18), 2436 (1994).

R. N. Compton and N. I. Hammer, in Advances in Gas-Phase Ion Chemstry, edited by N. G. Adams (JAI Press, Greenwich,CT, 2001), Vol. 4, pp. 257.

C. Desfrancois, Phys. Rev. A. 51 (5), 3667 (1995).

J. D. Power, Philos. Trans. R. Soc. London 274, 663 (1974).

B. L. Moiseiwitsch, Quantum Theory. (Academic Press, New York, 1961).

W. R. Garrett, J. Chem. Phys. 71, 651 (1979).

T. Koopmans, Physica (Amsterdam) 1, 104 (1934). 
M. Gutowski, P. Skurski, K. D. Jordan, and J. Simons, Int. J. Quantum Chem. 64, 183 (1997).

G. L. Gutsev and R. J. Bartlett, J. Chem. Phys. 105, 8785 (1996).

M. Gutowski and P. Skurski, J. Phys. Chem. B 101, 9143 (1997).

A. F. Jalbout and L. Adamowicz, J. Phys. Chem. A 105 (6), 1033 (2001).

A. F. Jalbout, C. S. Hall-Black, and L. Adamowicz, Chem. Phys. Lett. 354 (1-2), 128 (2002).

J. Schiedt, R. Weinkauf, D. M. Neumark, and E. W. Schlag, Chem. Phys. 239, 511 (1998).

R. N. Barnett, U. Landman, C. L. Cleveland, and J. Jortner, J. Chem. Phys. 88, 4421 (1988).

J. Schnitker and P. J. Rossky, J. Chem. Phys. 86, 3462 (1987).

A. Staib and D. Borgis, J. Chem. Phys. 103, 2642 (1995).

A. Wallqvist, D. Thirumalai, and B. J. Berne, J. Chem. Phys. 85, 1583 (1986).

D. C. Clary and D. M. Benoit, J. Chem. Phys. 111, 10559 (1999).

M. Sprik and M. L. Klein, J. Chem. Phys. 89, 7556 (1988).

H. Abdoul-Carime and C. Desfrancois, Eur. Phys. J. D 2 (2), 149 (1998).

A. Mosyak, P. Graf, I. Benjamin, and A. Nitzan, J. Phys. Chem. A 101, 429 (1996).

M. Rigby, E. B. Smith, W. A. Wakeham, and G. C. Maitland, The Forces

Between Molecules. (Clarendon, Oxford, 1986).

R. A. Kendall, T. H. Dunning, Jr, and R. J. Harrison, J. Chem. Phys. 96 (6796) (1992).

M. Gutowski (private communications)

P. J. Rossky (private communications)

The following procedure was used to determine the $\operatorname{CCSD}(\mathrm{T})$ charges: Charges were first fit using the QCISD electrostatic potential and dipole moments. They were then uniformly scaled so as to reproduce the $\operatorname{CCSD}(\mathrm{T})$ dipole moments. This results in a small change in the charges since the QCISD and CCSD(T) dipole moments are in close agreement.

Gaussian 98 (Revision A.9), M. J. Frisch, G. W. Trucks, H. B. Schlegel, G. E. Scuseria, M. A. Robb, J. R. Cheeseman, V. G. Zakrzewski, J. A. Montgomery, R. E. Stratmann, J. C. Burant, S. Dapprich, J. M. Millam, A. D. Daniels, K. N. Kudin, M. C. Strain, O. Farkas, J. Tomasi, V. Barone, M. Cossi, R. Cammi, B. Mennucci, C. Pomelli, C. Adamo, S. Clifford, J. Ochterski, G. A. Petersson, P. Y. Ayala, Q. Cui, K. Morokuma, D. K. Malick, A. D. Rabuck, K. Raghavachari, J. B. Foresman, J. Cioslowski, J. V. Ortiz, B. B. Stefanov, G. Liu, A. Liashenko, P. Piskorz, I. Komaromi, R. Gomperts, R. L. Martin, D. J. Fox, , T. Keith, M. A. AlLaham, C. Y. Peng, A. Nanayakkara, C. Gonzalez, M. Challacombe, P. M. W.

Gill, B. G. Johnson, W. Chen, M. W. Wong, J. L. Andres, M. Head-Gordon, E. S. Replogle, and J. A. Pople, Gaussian, Inc., Pittsburgh, PA, 1998.

R. Laenen, T. Roth, and A. Laubereau, Phys. Rev. Lett. 85, 50 (2000).

C. Silva, P. K. Walhout, K. Yokoyama, and P. F. Barbara, Phys. Rev. Lett. 80, 1086 (1998).

D. M. Bartels, A. R. Cook, M. Mudaliar, and C. D. Jonah, J. Phys. Chem. A 104

(8), 1686 (2000). 
R. A. Crowell and D. M. Bartels, J. Phys. Chem. 100, 17713 (1996).

D. M. Bartels, D. Gosztola, and C. D. Jonah, J. Phys. Chem. A 105, 8069 (2001).

J. V. Coe, Int. Rev. Phys. Chem. 20 (1), 33 (2001).

M. J. Tauber and R. A. Mathies, Chem. Phys. Lett. 354 (5-6), 518 (2002).

A. Staib and D. Borgis, J. Chem. Phys. 104, 9027 (1996).

W. Sheu and P. J. Rossky, Chem. Phys. Lett. 213, 233 (1993).

F. H. Long, H. Shi, K. Lu, and B. Eisenthal, J. Chem. Phys. 98, 7252 (1994).

Y. Gaudeul, H. Gelabert, and M. Ashokkumar, Chem. Phys. 197, 167 (1995).

J. A. Kloepfer, V. H. Vilchiz, V. A. Lenchenkov, and S. E. Bradforth, Chem.

Phys. Lett. 298, 120 (1998).

A. L. Sobolewski and W. Domcke, Phys. Chem. Chem. Phys. 4 (1), 4 (2002).

F. F. Muguet, H. Gelabert, and Y. Gaudeul, J. Chem. Phys. (93), 1808 (1996).

T. R. Tuttle, Jr. and S. Golden, J. Phys. Chem. 95, 5725 (1991).

E. J. Hart and M. Anbar, The Hydrated Electron. (Wiley-Interscience, New York, 1970).

L. Kevan, Acc. Chem. Res. 14 (5) (1981).

J. Schnitker and P. J. Rossky, J. Chem. Phys. 86, 3471 (1987).

P. Ayotte and M. A. Johnson, J. Chem. Phys. 106 (2), 811 (1997).

M. J. Tauber and R. A. Mathies, J. Phys. Chem. A 105, 10952 (2001).

H. Haberland, C. Ludewigt, H. Shindler, and R. Worsnop, Z. Phys. A 320, 151 (1985).

Y. Boutellier, C. Desfrancois, H. Abdoul-Carime, and J. P. Schermann, J. Chem. Phys. 105, 6420 (1996).

K. S. Kim, I. Park, S. Lee, K. Cho, J. Y. Lee, J. Kim, and J. D. Joannopoulos, Phys. Rev. Lett. 76 (6), 956 (1996).

J. V. Coe, J. Phys. Chem. A 101 (11), 2055 (1997).

T. Maeyama, T. Tsumura, A. Fujii, and N. Mikami, Chem. Phys. Lett. 264, 292 (1997).

J. Kim, I. Becker, O. Cheshnovsky, and M. A. Johnson, Chem. Phys. Lett. 297, 90 (1998).

D. M. A. Smith, J. Smets, Y. Elkadi, and L. Adamowicz, J. Chem. Phys. 109, 1238 (1998).

Y. V. Novakovskaya and N. F. Stepanov, Int. J. Quantum Chem. 88, 496 (2002).

H. Haberland, H. G. Schindler, R. Worsnop, and B. Bunsenges, Phys. Chem. 88, 271 (1984).

H. M. Lee, S. B. Suh, J. Y. Lee, P. Tarakeshwar, and K. S. Kim, J. Chem. Phys. 112 (22), 9759 (2000).

H. M. Lee, S. B. Suh, J. Y. Lee, P. Tarakeshwar, and K. S. Kim, J. Chem. Phys. 114 (7), 3343 (2001).

H. M. Lee, S. B. Suh, and K. S. Kim, J. Chem. Phys. 114 (24), 10749 (2001).

S. B. Suh, H. M. Lee, J. Kim, J. Y. Lee, and K. S. Kim, J. Chem. Phys. 113, 5273 (2000).

D. M. A. Smith, J. Smets, and L. Adamowicz, J. Chem. Phys. 110 (8), 3804 (1999).

H. M. Lee, S. B. Suh, and K. S. Kim, Bulletin of the Korean Chem. Soc. 21 (6), 555 (2000). 
C. G. Bailey, J. Kim, and M. A. Johnson, J. Phys. Chem. 100, 16782 (1996).

A. D. Becke, J. Chem. Phys. 98, 5648 (1993).

B. Mielich, A. Savin, H. Stoll, and H. Preuss, Chem. Phys. Lett. 157, 200 (1989).

C. Lee, W. Yang, and R. G. Parr, Phys. Rev. B. 37, 785 (1988).

M. Elstner, P. Hobza, T. Frauenheim, S. Suhai, and E. Kaxiras, J. Chem. Phys. 114 (12), 5149 (2001).

R. N. Barnett, U. Landman, D. Scharf, and J. Jortner, Acc. Chem. Res. 22, 350 (1989).

M. Marchi, M. Sprik, and M. L. Klein, J. Chem. Phys. 89, 4918 (1988).

U. Landman, R. N. Barnett, C. L. Cleveland, J. Luo, D. Scharf, and J. Jortner, in

Few Body Systems and Multiparticle Dynamics, edited by D. Micha (AIP, New York, 1987), pp. 200.

I. Benjamin, D. Evans, and A. Nitzan, J. Chem. Phys. 106, 6647 (1997).

R. N. Barnett, U. Landman, and A. Nitzan, J. Chem. Phys. 89, 2242 (1988).

R. N. Barnett, U. Landman, and A. Nitzan, Phys. Rev. Lett. 62, 106 (1989).

L. X. Dang and T. M. Chang, J. Chem. Phys. 106, 8149 (1997).

J. M. Pedulla and K. D. Jordan, Chem. Phys. 239, 593 (1998).

A. C. Shepard, Y. Beers, G. P. Klein, and L. S. Rothman, J. Chem. Phys. 59, 2254 (1973).

J. Verhoeven and A. Dymanus, J. Chem. Phys. 52, 3222 (1970).

F. H. Stillinger, in The Liquid State of Matter; Fluids, Simple and Complex, edited by E. W. Montroll and J. L. Lebowitz (North-Holland, Amsterdam, 1982), pp. 341.

D. R. Alfonso and K. D. Jordan, J. Chem. Phys. 116 (9), 3612 (2002).

These functions are the same functions as the two outermost functions in the $\mathrm{H}$ atom basis set used in the all-electron calculations.

S. T. Arnold, J. G. Eaton, D. Patel-Misra, H. W. Sarkas, and K. H. Bowen, Ion and Cluster Ion Spectroscopy and Structure. (Elsevier, Amsterdam, 1989).

S. T. Arnold, Ph.D. thesis, Johns Hopkins University, 1993.

Tsai, B and Jordan, KD. Unpublished results

E. J. Hart and J. W. Boag, J. Am. Chem. Soc. 84, 4090 (1962).

J. V. Coe, G. H. Lee, J. G. Eaton, S. T. Arnold, H. W. Sarkas, K. H. Bowen, C. Ludewigt, H. Haberland, and R. Worsnop, J. Chem. Phys. 92, 3980 (1990).

S. Lee, J. Kim, S. J. Lee, and K. S. Kim, Phys. Rev. Lett. 79 (11), 2038 (1997).

K. S. Kim, unpublished results

J. Simons and P. Skurski, in Theoretical Prospects of Negative Ions, edited by J.

Kalcher (Research Signpost, Trivandrum, 2002), pp. 117.

P. Skurski, M. Gutowski, and J. Simons, Int. J. Quantum Chem. 80 (4-5), 1024 (2000).

M. Gutowski and P. Skurski, J. Chem. Phys. 107, 2968 (1997).

J. P. Neirotti, F. Calvo, D. L. Freeman, and J. D. Doll, J. Chem. Phys. 112 (23), 10340 (2000).

A damping function that has the form of a constant minus a Gaussian is used here to facilitate evaluation of integrals.

C. J. Burnham and S. S. Xantheas, J. Chem. Phys. 116 (4), 1479 (2002). 
K. Makrodimitris, G. K. Papadopoulos, C. Philippopoulo, and D. N. Theodorou, J. Chem. Phys. 117 (12), 5876 (2002).

F. H. Stillinger and T. A. Weber, J. Phys. Chem. 87, 2833 (1983).

K. S. Kim, S. Lee, J. Kim, and J. Y. Lee, J. Am. Chem. Soc. 119, 9329 (1997). K. S. Kim, unpublished results

H. Abdoul-Carime, J. P. Schermann, and C. Desfrancois, Few-Body Systems 31, 183 (2002).

A. S. Mullin, K. K. Murray, C. P. Schulz, and W. C. Lineberger, J. Phys. Chem. 97 (40), 10281 (1993).

S. E. Novick, P. L. Jones, T. J. Mulloney, and W. C. Lineberger, J. Chem. Phys. 70 (5), 2210 (1979).

M. Gutowski, C. S. Hall, L. Adamowicz, J. H. Hendricks, H. L. de Clercq, S. A. Lyapustina, J. M. Nilles, S. J. Xu, and K. H. Bowen, Phys. Rev. Lett. 88 (14), 143001 (2002).

K. K. Sunil and K. D. Jordan, Chem. Phys. Lett. 164, 509 (1989).

P. Skurski and J. Simons, J. Chem. Phys. 116 (14), 6118 (2002).

P. Skurski, M. Gutowski, and J. Simons, Chem. Phys. Lett. 322, 175 (2000).

M. Gutowski, P. Skurski, and J. Simons, Int. J. Mass Spectrom 201, 245 (2000).

M. V. N. Ambika Prasad, R. F. Wallis, and R. Hermans, Phys. Rev. B. 40, 5924 (1989).

K. D. Jordan and J. F. Liebman, Chem. Phys. Lett. 62, 143 (1979).

M. Gutowski and P. Skurski, Chem. Phys. Lett. 303, 65 (1999).

M. V. N. Ambika Prasad, R. F. Wallis, and J. Hermans, Solid State Communications 77, 973 (1991).

G. L. Gutsev, R. J. Bartlett, and R. N. Compton, J. Chem. Phys. 108, 6756 (1998).

A. Halamaries, C. W. Walker, K. A. Simth, and F. B. Dunning, J. Chem. Phys. 89 (7226) (1988).

K. Harth, M. W. Ruf, and H. Z. Hotop, Z. Phys. D 14, 149 (1989).

K. D. Jordan and P. D. Burrow, Acc. Chem. Res. 11, 341 (1978).

C. Desfrancois, V. Periquet, S. Carles, J. P. Schermann, and L. Adamowicz, Chem. Phys. 239 (1-3), 475 (1998).

C. Desfrancois, H. Abdoul-Carime, and J. P. Schermann, J. Chem. Phys. 104 (7792) (1996).

K. R. Lykke, R. D. Mead, and W. C. Lineberger, Phys. Rev. Lett. 52 (25), 2221 (1984).

D. Wetzel and J. I. Brauman, J. Chem. Phys. 90 (1), 68 (1989).

R. D. Nelson, D. R. Lide, and A. A. Margott, Selected Values of Electric Dipole Moments for Molecules in Gas Phase. (Natl. Bur. Stand., Washington, D. C., 1967).

G. L. Gutsev and R. J. Bartlett, J. Chem. Phys. 105 (19), 8785 (1996).

A. Modelli and M. Venuti, Int. J. Mass Spectrom 205, 7 (2001).

T. Sommerfeld, Phys. Chem. Chem. Phys. 4, 2511 (2002).

C. Desfrancois, V. Periquet, Y. Boutellier, and J. P. Schermann, J. Phys. Chem. A 102 (8), 1274 (1998).

J. H. Hendricks, S. A. Lyapustina, H. L. d. Clercq, and K. H. Bowen, J. Chem. Phys. 108 (1), 8 (1998). 
K. Aflatooni, G. A. Gallup, and P. D. Burrow, J. Phys. Chem. 102, 6205 (1998). M. D. Sevilla, B. Besler, and A. O. Colson, J. Phys. Chem. 99, 1060 (1995). J. H. Hendricks, S. A. Lyapustina, H. L. de Clercq, and K. H. Bowen, J. Chem. Phys. 108 (1), 8 (1997). M. D. Sevilla, B. Besler, and A. O. Colson, J. Chem. Phys. 98, 2215 (1994). P. Skurski, J. Rak, and J. Simons, J. Chem. Phys. 115 (24), 11193 (2001). J. Rak, J. Blazejowski, and R. J. Zauhar, J. Organomet. Chem. 57 (3720) (1992). P. Jungwirth and V. Spirko, Phys. Rev. Lett. 84 (6), 1140 (2000).

P. Skurski, M. Gutowski, and J. Simons, Int. J. Quantum Chem. 76, 197 (1998). C. Sarasola, J. E. Fowler, and J. M. Ugalde, J. Chem. Phys. 110, 11717 (1999). C. Sarasola, J. E. Fowler, J. M. Elorza, and J. M. Ugalde, Chem. Phys. Lett. 337, 355 (2001).

P. Skurski and J. Simons, J. Chem. Phys. 112 (15), 6563 (2000).

E. A. Woronowic, W. H. Robertson, G. H. Weddle, E. M. Myshakin, K. D. Jordan, and M. A. Johnson, J. Phys. Chem. A 106 (31), 7086 (2002).

J. A. Weber, W. H. Kelly, M. A. Robertson, and M. A. Johnson, J. Chem. Phys. 114 (6), 2698 (2001).

E. M. Myshakin, W. H. Robertson, G. H. Weddle, K. D. Jordan, and M. A. Johnson, J. Chem. Phys. in press. (2002).

O. M. Cabarcos, C. J. Weinheimer, J. M. Lisy, and S. S. Xantheas, J. Chem. Phys. 110 (1), 5 (1999).

R. W. Schoenlein, J. G. Fujimoto, G. L. Eesley, and T. W. Capehat, Phys. Rev. Lett. 61, 2596 (1988).

P. M. Echenique and J. B. Pendry, Progress in Sur. Sci. 32, 111 (1989).

K. J. Taylor, C. Jin, J. Conceicao, L. Wang, O. Cheshnovsky, B. R. Johnson, P. J. Nordlander, and R. E. Smalley, J. Chem. Phys. 93 (10), 7515 (1990).

C. J. Martyna and B. J. Berne, J. Chem. Phys. 90, 3744 (1989).

H. Haberland and K. H. Bowen, in Clusters of atoms and molecules II. Solvation and chemistry of free clusters and embeded, supported and compressed clusters, edited by H. Haberland (Springer, Berlin, 1994), pp. 134.

P. Stampfli and K. Bennemann, Phys. Rev. A. 38, 4431 (1988).

H. Haberland, T. Kolar, and T. Reiners, Phys. Rev. Lett. 63 (12), 1219 (1989).

C. J. Martyna and B. J. Berne, J. Chem. Phys. 88 (7), 4516 (1988).

M. Rosenblit and J. Jortner, J. Phys. Chem. 98, 9365 (1994).

J. O. Jung and R. B. Gerber, J. Chem. Phys. 105, 10322 (1996).

P. Skurski and M. Gutowski, J. Chem. Phys. 108, 6303 (1998).

Y. Boutellier, C. Desfrancois, H. Abdoul-Carime, and S. J. P., J. Chem. Phys. 105, 6420 (1996).

J. H. Hendricks, H. L. de Clercq, S. A. Lyapustina, and K. H. Bowen, J. Chem. Phys. 107, 2962 (1997).

H. J. C. Berendsen, J. P. M. Postma, W. F. Van Gunsteren, and J. Hermans, in Intermolecular Forces, edited by B. Pullman (Reidel, Dordrecht, 1981), pp. 331. J. R. Reimers and R. O. Watts, Chem. Phys. 85, 83 (1984).

H. L. Lemberg and F. H. Stillinger, J. Chem. Phys. 62, 1677 (1975).

L. X. Dang, J. Chem. Phys. 97, 2659 (1992). 


\section{Tables}


Table I: Contributions to the vertical detachment energy $\left(\mathrm{cm}^{-1}\right)$ of the dipole-bound anions of selected polar molecules and their clusters as described by ab initio calculations. ${ }^{\text {a }}$

\begin{tabular}{|c|c|c|c|c|c|c|c|c|c|c|c|}
\hline System & $\mu_{\mathrm{HF}}(\mathrm{D})$ & $\mu_{\text {corr }}(\mathrm{D})$ & $\mathbf{E A}^{\mathrm{KT}}$ & $\Delta \mathbf{E}^{\text {relax }}$ & $\Delta \mathbf{E}^{(2)-d i s p}$ & $\Delta \mathbf{E}^{(2)-n o n d i s p}$ & $\Delta \mathbf{E}^{(3)}$ & $\Delta \mathbf{E}^{(4)}$ & $\Delta \mathbf{E}^{\mathrm{HO}}$ & $\mathbf{E}^{\text {total }}$ & $\mathbf{E A}^{\operatorname{expt} \mathbf{j}}$ \\
\hline $\mathrm{HCN}^{-b}$ & 3.29 & $3.05^{\mathrm{h}}$ & 11.2 & 0.4 & 11.2 & -10.9 & -0.1 & 1.2 & -0.2 & 13.2 & \\
\hline$(\mathrm{HCN})_{2}^{-\mathrm{c}}$ & 7.60 & $6.88^{h}$ & 483 & 49 & 215 & -232 & 15 & 17 & -17 & 530 & \\
\hline$\left(\mathrm{H}_{2} \mathrm{O}\right)_{2}^{-\mathrm{d}}$ & 4.41 & $4.18^{i}$ & 111 & 7 & 114 & -10 & 0 & 20 & 100 & 312 & $363 \pm 48^{\mathrm{k}}$ \\
\hline$(\mathrm{HF})_{2}{ }^{-\mathrm{e}}$ & 3.98 & $3.78^{i}$ & 165 & 14 & 177 & -73 & -3 & 27 & 81 & 387 & $508 \pm 24^{1, \mathrm{~m}}$ \\
\hline$(\mathrm{HF})_{3}^{-\mathrm{f}}$ & 6.54 & $6.27^{\mathrm{i}}$ & 950 & 104 & 625 & -227 & -24 & 93 & 145 & 1666 & $1613-2420^{d}$ \\
\hline $\mathrm{CH}_{3} \mathrm{CN}^{-\mathrm{g}}$ & 4.34 & $3.94^{\mathrm{i}}$ & 53 & 3 & 57 & -38 & 4 & 8 & 22 & 108 & $145-150^{\mathrm{n}}$ \\
\hline
\end{tabular}

$\overline{{ }^{a}}$ The vertical detachment energies are the negatives of the electron binding energies. The second-order dispersion and non-dispersion contribution are estimated using a procedure of Gutowski and coworkers. ${ }^{23,26,70}$ The $\Delta \mathrm{E}^{\mathrm{HO}}$ contributions are obtained by comparing the results of MP4 and CCSDT calculations for HCN and MP4 and CCSD(T) calculations for the other systems.

${ }^{\mathrm{b} 32} ;{ }^{\text {c } 27} ;{ }^{\text {d 26}} ;{ }^{\text {e } 150} ;{ }^{70} ;{ }^{\text {g 209. }}{ }^{\text {h }}$ MP2 dipole; ${ }^{\text {i }}$ QCISD dipole.

${ }^{\mathrm{j}}$ Experimental vertical detachment energies.

${ }^{\mathrm{k}}{ }^{141} ;{ }^{1}$ A separate experiment ${ }^{210}$ gave a VDE of $242 \pm 32$ for $(\mathrm{HF})_{2}{ }^{-} ;{ }^{\mathrm{m} 211} ;{ }^{\mathrm{n}} 63$. 
Table II. Parameters used in the model potentials for $\mathrm{HCN},(\mathrm{HCN})_{2}, \mathrm{HNC}$ and $(\mathrm{HNC})_{2}{ }^{\mathrm{a}}$.

\begin{tabular}{|c|c|c|c|c|c|c|c|c|}
\hline \multirow{3}{*}{$\begin{array}{l}\text { Molecule } \\
\text { HCN, } \\
(\mathrm{HCN})_{2}\end{array}$} & \multicolumn{2}{|c|}{$\begin{array}{l}\text { Bond length } \\
(\AA)\end{array}$} & \multicolumn{2}{|c|}{$\begin{array}{c}\text { Monomer- } \\
\text { polarizability }\left(\mathrm{a}_{0}{ }^{3}\right)^{\mathrm{b}}\end{array}$} & \multicolumn{2}{|c|}{ "HF charges } & \multicolumn{2}{|c|}{$\begin{array}{l}\operatorname{CCSD}(\mathrm{T}) \\
\text { charges }\end{array}$} \\
\hline & $\mathrm{R}_{\mathrm{HC}}$ & 1.078 & $\alpha_{x x}$ & 14.24 & $\mathrm{Q}_{\mathrm{H}}$ & 0.269 & $\mathrm{Q}_{\mathrm{H}}$ & 0.223 \\
\hline & $\mathrm{R}_{\mathrm{CN}}$ & 1.183 & $\alpha_{\mathrm{yy}}$ & 14.24 & $\mathrm{Q}_{\mathrm{C}}$ & 0.071 & $\mathrm{Q}_{\mathrm{C}}$ & 0.103 \\
\hline & $\mathrm{R}_{12}$ & 2.183 & $\alpha_{\mathrm{zz}}$ & 24.63 & $\mathrm{Q}_{\mathrm{N}}$ & -0.340 & $\mathrm{Q}_{\mathrm{N}}$ & -0.326 \\
\hline \multirow{3}{*}{$\begin{array}{l}\mathrm{HNC} \\
(\mathrm{HNC})_{2}\end{array}$} & $\mathrm{R}_{\mathrm{HN}}$ & 0.995 & $\alpha_{x x}$ & 15.68 & $\mathrm{Q}_{\mathrm{H}}$ & 0.315 & $\mathrm{Q}_{\mathrm{H}}$ & 0.293 \\
\hline & $\mathrm{R}_{\mathrm{NC}}$ & 1.170 & $\alpha_{\mathrm{yy}}$ & 15.68 & $\mathrm{Q}_{\mathrm{N}}$ & -0.073 & $\mathrm{Q}_{\mathrm{N}}$ & 0.011 \\
\hline & $\mathrm{R}_{12}$ & 2.076 & $\alpha_{\mathrm{zz}}$ & 23.53 & $\mathrm{Q}_{\mathrm{C}}$ & -0.242 & $\mathrm{Q}_{\mathrm{C}}$ & -0.304 \\
\hline
\end{tabular}

$\overline{\overline{\text { a.The origins }}} \overline{\overline{\text { for the Drude oscillators are located on }}} \overline{\overline{ }} \overline{\overline{\text { the } \mathrm{CN}}} \overline{\overline{\mathrm{N} \text { groups } 2}} \overline{\overline{\mathrm{Bohr}}} \overline{\overline{\text { from } \mathrm{H}}}$ atoms.

${ }^{b}$ Polarizabilities calculated at the MP2/aug-cc-pVTZ level of theory. 
Table III. Excess electron binding energies for $\mathrm{HCN}, \mathrm{HNC},(\mathrm{HCN})_{2}$ and $(\mathrm{HNC})_{2}{ }^{a}$

\begin{tabular}{|c|c|c|c|c|c|c|c|c|c|}
\hline \multirow[t]{2}{*}{ Molecule } & \multirow[t]{2}{*}{ "Approach } & \multirow[t]{2}{*}{$\bar{~} \mu$ (Debye) } & \multicolumn{7}{|c|}{ Contributions to binding energy $\left(\mathrm{cm}^{-1}\right)$} \\
\hline & & & KT & Polariz. & $\begin{array}{l}\text { Disp. } \\
2^{\text {nd }} \text { order }\end{array}$ & $\begin{array}{l}\text { Non- } \\
\text { disp. } 2^{\text {nd }} \\
\text { order }\end{array}$ & $\begin{array}{l}\text { Total } \\
\text { through } \\
2^{\text {nd }} \text { order }\end{array}$ & $\begin{array}{l}\text { Higher } \\
\text { order }\end{array}$ & $\begin{array}{l}\text { Net } \\
\text { binding }\end{array}$ \\
\hline \multirow[t]{3}{*}{$\mathrm{HCN}$} & $a b$ initio & $\begin{array}{l}3.33(\mathrm{HF}) \\
3.01(\mathrm{CCSD}(\mathrm{T}))\end{array}$ & -11.7 & -0.47 & -13.0 & 12.5 & -12.7 & -0.5 & $\begin{array}{l}-13.2 \\
(-9.0)\end{array}$ \\
\hline & Model I & 3.32 & -11.7 & -0.48 & -11.0 & & -23.2 & -10.5 & -33.7 \\
\hline & Model II & 3.01 & -3.50 & -0.08 & -3.67 & & -7.25 & -5.0 & -12.3 \\
\hline \multirow[t]{3}{*}{$\mathrm{HNC}$} & ab initio & $\begin{array}{l}\text { 2.87(HF) } \\
3.11(\mathrm{CCSD}(\mathrm{T}))\end{array}$ & -2.72 & -0.06 & -4.13 & -5.0 & -11.9 & -23.8 & $\begin{array}{l}-35.7 \\
(-41.3)\end{array}$ \\
\hline & Model I & 2.87 & -2.72 & -0.06 & -3.60 & & -6.4 & -6.7 & -13.1 \\
\hline & Model II & 3.11 & -7.53 & -0.29 & -8.92 & & -16.7 & -12.6 & -29.3 \\
\hline \multirow[t]{5}{*}{$(\mathrm{HCN})_{2}$} & $a b$ initio & $\begin{array}{l}\text { 7.54(HF) } \\
6.85(\mathrm{CCSD}(\mathrm{T}))\end{array}$ & -461 & -52 & -222 & 211 & -524 & -1 & $(-525)$ \\
\hline & Model I & 7.24 & -405 & -40 & -135 & & -581 & -41 & -622 \\
\hline & Model II & 6.55 & -259 & -24 & -103 & & -386 & -41 & -427 \\
\hline & Model III & 7.54 & -475 & -48 & -147 & & -670 & -41 & -711 \\
\hline & Model IV & 6.85 & -315 & -30 & -117 & & -463 & -41 & -504 \\
\hline \multirow[t]{5}{*}{$(\mathrm{HNC})_{2}$} & $a b$ initio & $\begin{array}{l}\text { 6.70(HF) } \\
7.31(\mathrm{CCSD}(\mathrm{T}))\end{array}$ & -366 & -43 & -244 & -217 & -870 & -93 & $(-963)$ \\
\hline & Model I & 6.31 & -291 & -32 & -127 & & -450 & -48 & -498 \\
\hline & Model II & 6.85 & -396 & -44 & -151 & & -591 & -48 & -639 \\
\hline & Model III & 6.71 & -383 & -43 & -148 & & -574 & -47 & -621 \\
\hline & Model IV & 7.31 & -516 & -57 & -172 & & -746 & -46 & -792 \\
\hline
\end{tabular}


${ }^{a}$ Models I and II employ monomer atomic charges from HF and CCSD(T) calculations, respectively, For the dimers, these models also include the effect of the lowest-order induced dipole moments. The induced dipoles in models III and IV have been scaled so that the model potentials give the same net dipole moments for the dimers as obtained from HF and CCSD(T) calculations, respectively.

${ }^{b}$ When reporting net $a b$ initio binding energies, CCSDT results are used where available, the CCSD(T) electron binding energies are put in parentheses. 
Table IV. Characteristics of various one-electron models for describing negatively charged clusters of water molecules or an excess electron in bulk water.

\begin{tabular}{|c|c|c|}
\hline Potential & Water-water ${ }^{\mathrm{a}, \mathrm{b}}$ & Electron-water \\
\hline $\begin{array}{l}\text { Schnitker- } \\
\text { Rossky }^{75}\end{array}$ & $\begin{array}{l}\text { SPC } \\
=212, \text { rigid, non-polarizable, } \mu \\
=27 \mathrm{D}\end{array}$ & 2-body polarizable, ${ }^{\mathrm{c}}-\frac{\alpha}{2 r^{4}}\left[1-e^{-\left(r / r_{c}\right)^{6}}\right]$ \\
\hline Barrett- & RWK2-M ${ }^{213}$, flexible, non- & $\begin{array}{lll}- & \alpha\end{array}$ \\
\hline Staib-Borgis $^{76}$ & $\begin{array}{l}\text { pTIP4P }{ }^{79} \text {, rigid, polarizable via } \\
\text { fluctuating charge, } \mu=1.85 \mathrm{D}\end{array}$ & $\begin{array}{l}\text { fluctuating charge, self consistent } \\
\text { polarizable }\end{array}$ \\
\hline $\begin{array}{l}\text { Wallqvist - } \\
\text { Berne }^{77}\end{array}$ & $\begin{array}{l}\text { modified central force } \\
\text { potential }^{214} \text {, flexible, non- } \\
\text { polarizable, } \mu=1.86 \mathrm{D}\end{array}$ & 2-body polarizable, $-\frac{\alpha}{2 r^{4}}\left[1-e^{-\left(r / r_{c}\right)^{12}}\right]$ \\
\hline Mosyak, et al. $^{81}$ & $\begin{array}{l}\text { pfSPC }^{81,215} \text {, flexible, polarizable, } \\
\mu=2.02 \mathrm{D}\end{array}$ & $\begin{array}{l}\text { self-consistent polarizable, } \\
-\frac{1}{2} \sum \mu_{j} \cdot E_{j}, \text { with }-\frac{1}{2} \frac{\alpha}{\left[\left|\vec{r}-\overrightarrow{R_{j}}\right|^{2}+R_{c}^{2}\right]^{2}}\end{array}$ \\
\hline Wang-Jordan ${ }^{34}$ & $\begin{array}{l}\text { Dang-Chang }{ }^{134} \text {, rigid, } \\
\text { polarizable, } \mu=1.85 \mathrm{D}\end{array}$ & $\begin{array}{l}\text { self-consistent polarization and dispersion } \\
\text { via Drude oscillators }\end{array}$ \\
\hline
\end{tabular}

${ }^{\mathrm{a}}$ Models with fixed monomer geometries are designated as rigid, and those in which the monomer geometries can relax are designated as flexible.

${ }^{\mathrm{b}}$ The dipole moments are for the isolated monomers.

${ }^{\mathrm{c}}$ Two-body polarizable models allow the excess electron to polarize the water molecules, (with polarizability centered on $\mathrm{O}$ ), but neglect couplings between the induced dipoles. Models designated as self-consistent polarizable treat electron-water and water-water polarization self-consistently.

${ }^{\mathrm{d}}$ The polarizable water model of Mosyak et al. introduces a polarizable site with polarizability $\alpha=1.44 \AA^{3}$ at the position of the $\mathrm{O}$ atom.

${ }^{\mathrm{e}}$ The polarizable center is on the $M$ site, which is on the $\mathrm{C}_{2}$ axis, $0.215 \AA$ from the oxygen atom, displaced toward the $\mathrm{H}$ atoms. ${ }^{34}$ 
Table V. Diffuse gaussian functions used in calculations on the $\left(\mathrm{H}_{2} \mathrm{O}\right)_{\mathrm{n}}{ }^{-}$clusters.

\begin{tabular}{|c|c|c|c|c|c|c|c|}
\hline \multicolumn{2}{|c|}{$\left(\mathrm{H}_{2} \mathrm{O}\right)_{2}^{-}$} & \multicolumn{2}{|c|}{$\left(\mathrm{H}_{2} \mathrm{O}\right)_{3}{ }^{-}$} & \multicolumn{2}{|c|}{ Crown $\left(\mathrm{H}_{2} \mathrm{O}\right)_{4}^{-}$} & \multicolumn{2}{|c|}{ Chain $\left(\mathrm{H}_{2} \mathrm{O}\right)_{4}^{-}$} \\
\hline$s$ & $p$ & $s$ & $p$ & $s$ & $p$ & $s$ & $p$ \\
\hline $2.5 \times 10^{-2}$ & $6.0 \times 10^{-2}$ & $7.5 \times 10^{-2}$ & $6.0 \times 10^{-2}$ & $3.0 \times 10^{-2}$ & $3.0 \times 10^{-2}$ & $3.0 \times 10^{-2}$ & $3.0 \times 10^{-2}$ \\
\hline $5.0 \times 10^{-3}$ & $1.2 \times 10^{-2}$ & $1.5 \times 10^{-2}$ & $1.2 \times 10^{-2}$ & $6.0 \times 10^{-3}$ & $6.0 \times 10^{-3}$ & $6.0 \times 10^{-3}$ & $6.0 \times 10^{-3}$ \\
\hline $1.0 \times 10^{-3}$ & $2.4 \times 10^{-3}$ & $3.0 \times 10^{-3}$ & $2.4 \times 10^{-3}$ & $1.2 \times 10^{-3}$ & $1.2 \times 10^{-3}$ & $1.2 \times 10^{-3}$ & $1.2 \times 10^{-3}$ \\
\hline $2.0 \times 10^{-4}$ & $4.8 \times 10^{-4}$ & $6.0 \times 10^{-4}$ & $4.8 \times 10^{-4}$ & $2.4 \times 10^{-4}$ & $2.4 \times 10^{-4}$ & $2.4 \times 10^{-4}$ & \\
\hline $4.0 \times 10^{-5}$ & $9.6 \times 10^{-5}$ & $1.2 \times 10^{-4}$ & $9.6 \times 10^{-5}$ & $4.8 \times 10^{-5}$ & & & \\
\hline & & $2.4 \times 10^{-5}$ & & & & & \\
\hline
\end{tabular}


Table VI. Electron binding energies (meV) for the water dimer. ${ }^{\text {a }}$

\begin{tabular}{crlcll}
\hline \hline Method & \multicolumn{5}{c}{ Energy contribution } \\
\cline { 2 - 6 } & $\mathrm{KT}$ & Polariz. & Disp. & $\mathrm{MP}^{\mathrm{b}}$ & $\mathrm{CCSD}(\mathrm{T}) / \mathrm{CI}^{\mathrm{c}}$ \\
\hline ab initio & 11.1 & 0.6 & 12.0 & 19.7 & 33.0 \\
Model I & 11.1 & 0.6 & 11.1 & 22.8 & 40.0 \\
Model II & 6.4 & 0.3 & 7.9 & 14.7 & 33.0 \\
\hline \hline
\end{tabular}

${ }^{\mathrm{a}}$ scaling factor 6.8, damping factor $b=0.43$.

${ }^{\mathrm{b}} \mathrm{MP} 2$ denotes the electron binding energies calculated through second-order, and which sum the KT, polarization (Polariz.), second-order dispersion (Disp.), second-order nondispersion contributions.

${ }^{\mathrm{c}}$ This column reports electron binding energies obtained from $a b$ initio $\operatorname{CCSD}(\mathrm{T})$ and model-potential CI calculations, 
Table VII. Electron binding energies (meV) of the water trimer.

\begin{tabular}{clrlrrr}
\hline \hline Structure & Method & \multicolumn{5}{c}{ Energy contribution } \\
\cline { 3 - 7 } & & KT & Polariz. & Disp. & MP2 $^{\mathrm{a}}$ & CCSD(T)/CI \\
\hline Crown & ab initio & 3.3 & 0.1 & 4.0 & 5.7 & 13.0 \\
& Model I & 3.3 & 0.1 & 3.9 & 7.3 & 17.4 \\
& Model II & 1.4 & 0.1 & 2.2 & 3.7 & 13.6 \\
Transition & ab initio & 34.8 & 3.3 & 31.6 & 59.8 & 87.0 \\
State & Model I & 34.8 & 3.0 & 28.7 & 66.6 & 101.7 \\
& Model II & 23.2 & 2.1 & 23.6 & 48.9 & 89.5 \\
Chain & ab initio & 60.7 & 4.4 & 43.8 & 97.1 & 127.0 \\
& Model I & 60.4 & 4.3 & 40.6 & 105.3 & 142.1 \\
& Model II & 45.0 & 3.5 & 36.5 & 85.0 & 128.6 \\
\hline \hline
\end{tabular}

\footnotetext{
${ }^{a}$ MP2 denotes the electron binding energies calculated through second-order, and which sum the KT, polarization (Polariz.), second-order dispersion (Disp.), second-order nondispersion contributions.

${ }^{\mathrm{b}}$ This column reports electron binding energies obtained from ab initio $\operatorname{CCSD}(\mathrm{T})$ and model-potential CI calculations.
} 
Table VIII. Electron binding energies (meV) of the water tetramer.

\begin{tabular}{llrlrrr}
\hline Structure & Method & \multicolumn{5}{c}{ Energy Contribution } \\
\cline { 3 - 7 } & & \multicolumn{1}{c}{ KT } & Polariz. & Disp. & \multicolumn{1}{c}{ MP2 $^{\mathrm{a}}$} & \multicolumn{1}{c}{ CCSD(T)/CI } \\
\hline \multirow{7}{*}{ Crown } & ab initio & 12.5 & 0.8 & 12.1 & 20.9 & 36.5 \\
& Model I & 12.9 & 0.9 & 11.7 & 25.6 & 46.6 \\
& Model II & 7.3 & 0.5 & 8.5 & 16.3 & 40.0 \\
Chain & ab initio & 110.2 & 8.2 & 67.7 & 170.3 & 209.6 \\
& Model I & 109.2 & 8.1 & 62.6 & 179.9 & 227.2 \\
& Model II & 87.8 & 7.3 & 59.7 & 154.7 & 212.5 \\
\hline \hline
\end{tabular}

${ }^{\mathrm{a}} \mathrm{MP} 2$ denotes the electron binding energies calculated through second-order, and which sum the KT, polarization (Polariz.), second-order dispersion (Disp.), second-order nondispersion contributions.

${ }^{\mathrm{b}}$ This column reports electron binding energies obtained from ab initio $\operatorname{CCSD}(\mathrm{T})$ and model-potential CI calculations. 
Table IX. Comparison of electron binding energies (meV) from MP2 calculations with model III, CI calculations with model II, and $a b$ initio calculations.

\begin{tabular}{cccllll}
\hline \hline Methods & \multicolumn{6}{c}{ Energy Contribution } \\
\cline { 2 - 7 } & $\begin{array}{c}\left(\mathrm{H}_{2} \mathrm{O}\right)_{2} \\
\text { chain }\end{array}$ & $\begin{array}{l}\left(\mathrm{H}_{2} \mathrm{O}\right)_{3} \\
\text { crown }\end{array}$ & $\begin{array}{l}\left(\mathrm{H}_{2} \mathrm{O}\right)_{3} \\
\text { TS }\end{array}$ & $\begin{array}{l}\left(\mathrm{H}_{2} \mathrm{O}\right)_{3} \\
\text { chain }\end{array}$ & $\begin{array}{l}\left(\mathrm{H}_{2} \mathrm{O}\right)_{4} \\
\text { crown }\end{array}$ & $\begin{array}{l}\left(\mathrm{H}_{2} \mathrm{O}\right)_{4} \\
\text { chain }\end{array}$ \\
\hline $\begin{array}{c}\text { ab initio } \\
\text { CCSD(T) }\end{array}$ & 33.0 & 13.0 & 87.0 & 127.0 & 36.5 & 209.6 \\
$\begin{array}{c}\text { Model II } \\
\text { CI }\end{array}$ & 33.0 & 14.0 & 89.8 & 127.8 & 40.5 & 210.8 \\
$\begin{array}{c}\text { Model III } \\
\text { MP2 }\end{array}$ & 34.8 & 10.5 & 91.9 & 142.5 & 33.7 & 236.5 \\
\hline \hline
\end{tabular}


Table X. Electron binding energies (in $\mathrm{meV}$ ) and total energies relative to dbs4dbs2 isomer (in kcal/mol) calculated for selected $\left(\mathrm{H}_{2} \mathrm{O}\right)_{6}{ }^{-}$isomers. ${ }^{\mathrm{a}}$, b

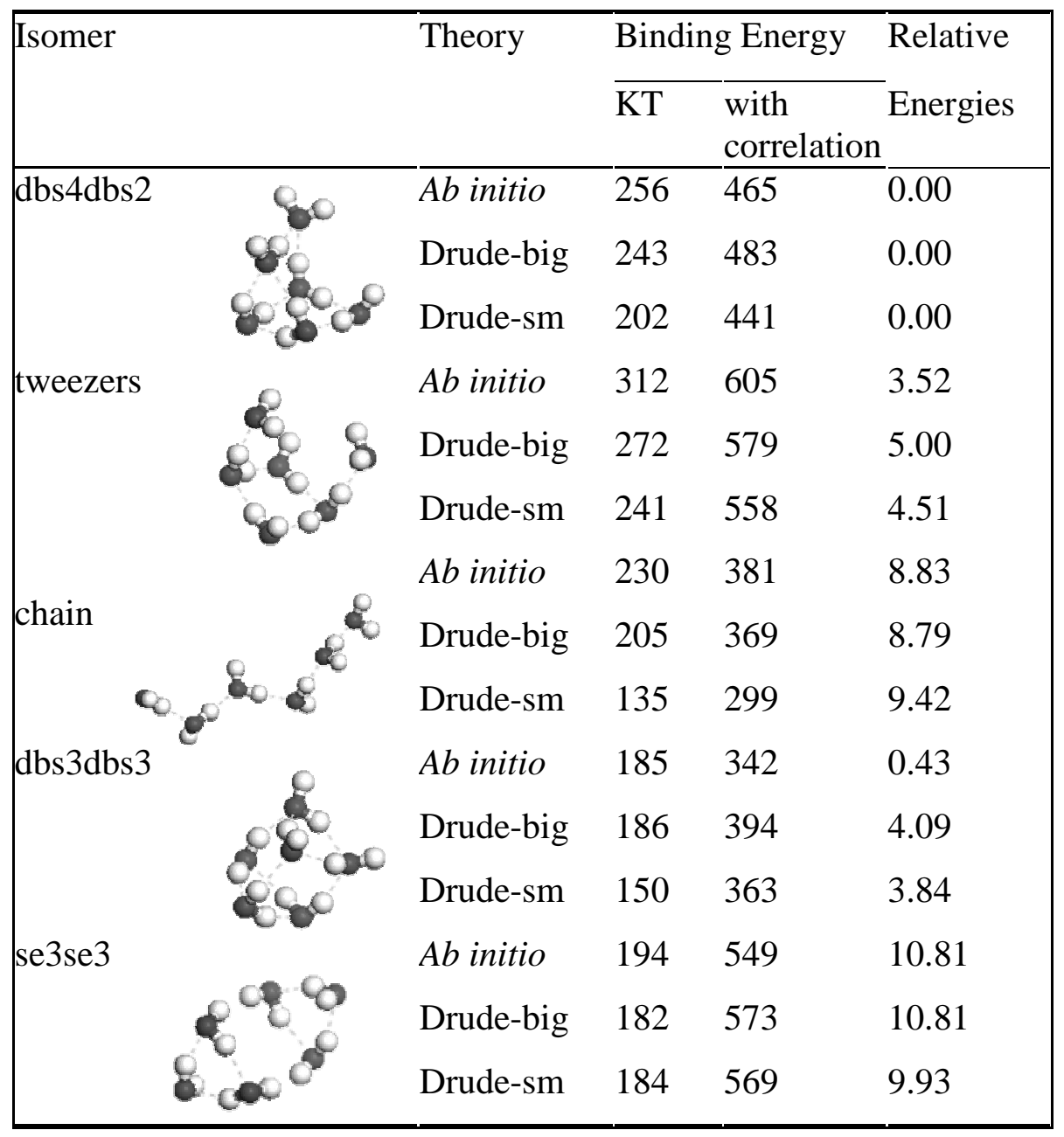

${ }^{\mathrm{a}}$ The $a b$ initio electron binding energies are from Ref. 54. The results including electron correlation were obtained at the $\operatorname{CCSD}(\mathrm{T})$ level of theory.

${ }^{\mathrm{b}}$ Drude model calculations used geometries from Ref. 4 adjusted so that the water monomers were restored to the geometries of an isolated, undistorted monomer. The two sets of Drude model results differ in terms of the size and location of the large singlecenter expansion of diffuse $s$ and $p$ functions. The Drude-big calculations use a large $8 s 5 p$ single-center expansion, the center of which was optimized. The Drude-sm calculations used the smaller $5 s 4 p$ single-center expansion described in the text and located at the center of mass. 
Table XI. $J_{z}$ values for selected isomers of $\left(\mathrm{H}_{2} \mathrm{O}\right)_{6}{ }^{-}$

\begin{tabular}{ll}
\hline Isomer $^{\mathrm{a}}$ & $J_{z}$ values \\
\hline prism; dbs4dbs2 & $6.0 \leq J_{z} \leq 7.5$ \\
cage & $7.5 \leq J_{z} \leq 8.5$ \\
open-book & $8.5 \leq J_{z} \leq 11.0$ \\
cage+tail & $8.5 \leq J_{z} \leq 10.0$ \\
ring & $9.0 \leq J_{z} \leq 10.0$ \\
ring plus tail(s) & $11.0 \leq J_{z} \leq 16.0$ \\
chain & $J_{z} \geq 16$ \\
\hline${ }^{\text {a }}$ The various structures are defined in Fig. 1
\end{tabular}


Table XII, Number of configurations with dbs4dbs2, dbs4'dbs2, and tweezers structures out of a total of 3997 configurations saved for each temperature in the parallel tempering Monte-Carlo simulations of $\left(\mathrm{H}_{2} \mathrm{O}\right)_{6}{ }^{-}$.

\begin{tabular}{llll}
\hline \hline \multirow{2}{*}{$\begin{array}{c}\text { Temperature } \\
\text { K })\end{array}$} & \multicolumn{3}{c}{ Target configuration } \\
\cline { 2 - 4 } & dbs4dbs2 & dbs4'dbs2 & tweezers \\
\hline 190.0 & 3 & 1 & 0 \\
157.0 & 20 & 2 & 0 \\
129.7 & 15 & 2 & 0 \\
107.2 & 11 & 3 & 0 \\
88.6 & 5 & 2 & 0 \\
73.2 & 7 & 0 & 0 \\
60.5 & 3 & 0 & 0 \\
50.0 & 0 & 0 & 0 \\
\hline \hline
\end{tabular}


Table XIII: Electron binding energies (meV) of the $(\mathrm{HF})_{n}, n=2-6$, solvated-electron species. $^{\text {a }}$

\begin{tabular}{lllllllll}
\hline Species & symm. & $\mathbf{E A}^{\mathbf{K T}}$ & $\Delta \mathbf{E}^{\text {relax }}$ & $\Delta \mathbf{E}^{(2)-d i s p}$ & $\Delta \mathbf{E}^{(2)-n o n d i s p}$ & $\Delta \mathbf{E}^{(3)}$ & $\Delta \mathbf{E}^{(4+H O)}$ & $\mathbf{E A}^{\text {total }}$ \\
\hline$(\mathrm{HF})_{2}$ & $\mathrm{D}_{\infty \mathrm{h}}$ & 12.3 & 3.5 & 42.0 & -16.7 & -0.5 & 12.8 & 53.4 \\
$(\mathrm{HF})_{3}$ & $\mathrm{D}_{3 \mathrm{~h}}$ & 243.8 & 158.9 & 427.1 & -211.6 & -17.4 & 32.1 & 632.9 \\
$(\mathrm{HF})_{4}$ & $\mathrm{~T}_{\mathrm{d}}$ & 807.9 & 404.4 & 685.4 & -427.3 & -26.1 & 32.2 & 1476.5 \\
$(\mathrm{HF})_{5}$ & $\mathrm{D}_{3 \mathrm{~h}}$ & 1357.9 & 497.3 & 753.6 & -513.1 & -21.8 & 26.0 & 2099.9 \\
$(\mathrm{HF})_{6}$ & $\mathrm{O}_{\mathrm{h}}$ & 1930.7 & 556.2 & 796.6 & -584.1 & -18.0 & 24.0 & 2691.2 \\
\hline
\end{tabular}

${ }^{\text {a) }}(\mathrm{HF})_{2}$ and $(\mathrm{HF})_{3}$ results from Ref. 70 .

b) $(\mathrm{HF})_{3},(\mathrm{HF})_{4}$, and $(\mathrm{HF})_{5}$ results from Ref. 84. 


\section{Figures}

Figure 1. Energies of an electron in the potential due to a finite dipole with $Q=1$. The energies are calculated using a large set of primitive $s, p, d$, and $f$ Gaussian-type functions centered on the positive charge. Energies less than about $10^{-4} \mathrm{eV}$ are unreliable due to the limitations of the basis set.

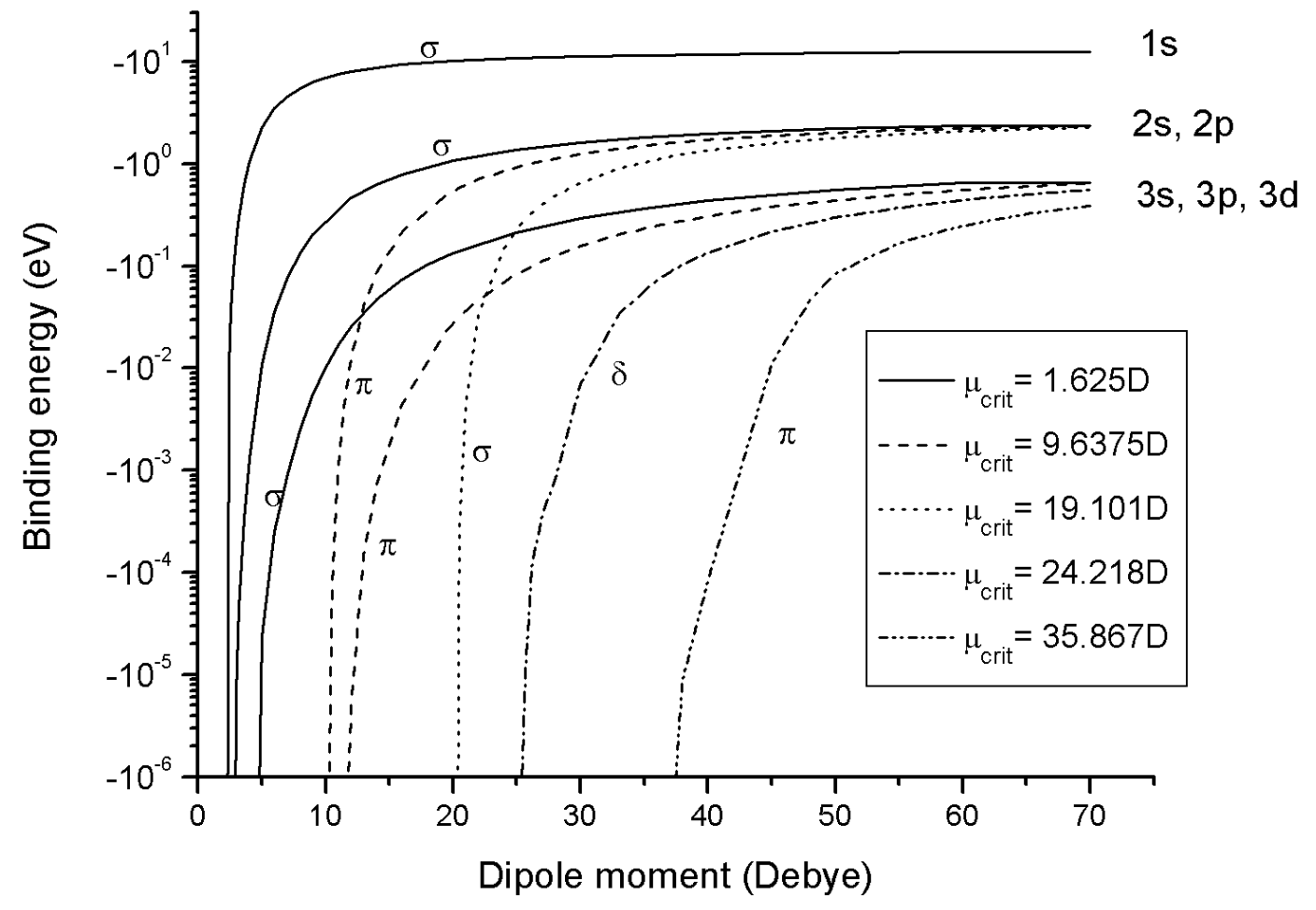


Figure 2. Charge distributions of an excess electron bound to a finite dipole: (a) $Q=1, \mu$ $=3$ and $6 \mathrm{D}$, no repulsive core, (b) $Q=0.5, \mu=3$ and $6 \mathrm{D}$, no repulsive core, (c) $Q=1, \mu$ $=3$ and $6 \mathrm{D}$, repulsive core, and (d) $Q=0.5, \mu=3$ and $6 \mathrm{D}$, repulsive core. Binding energies are also reported.
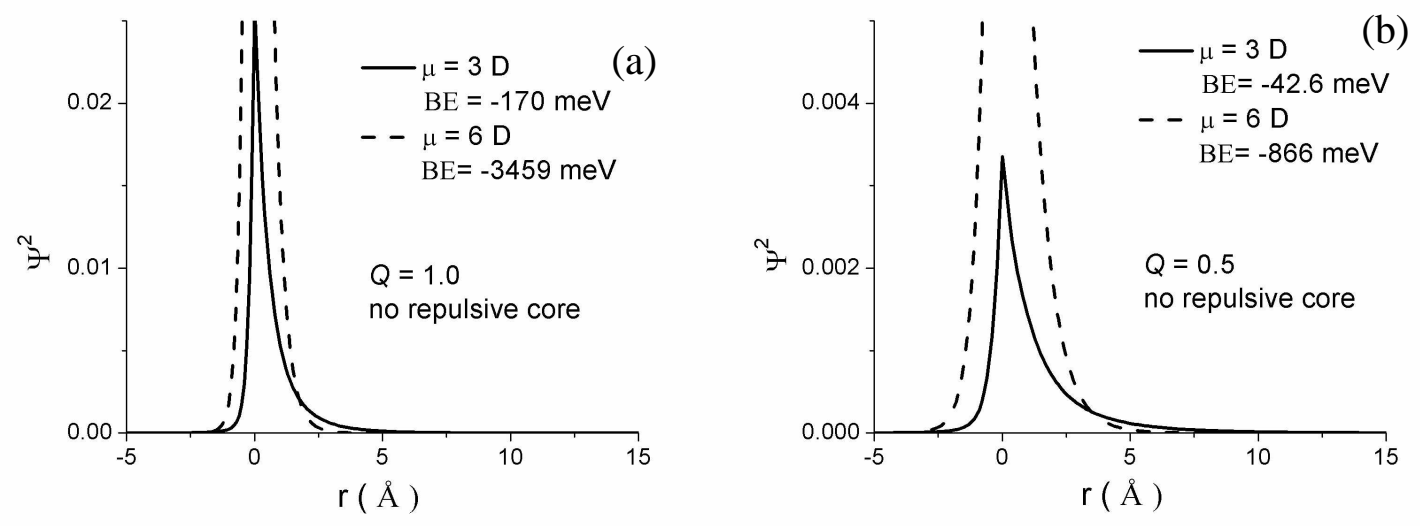

(c)
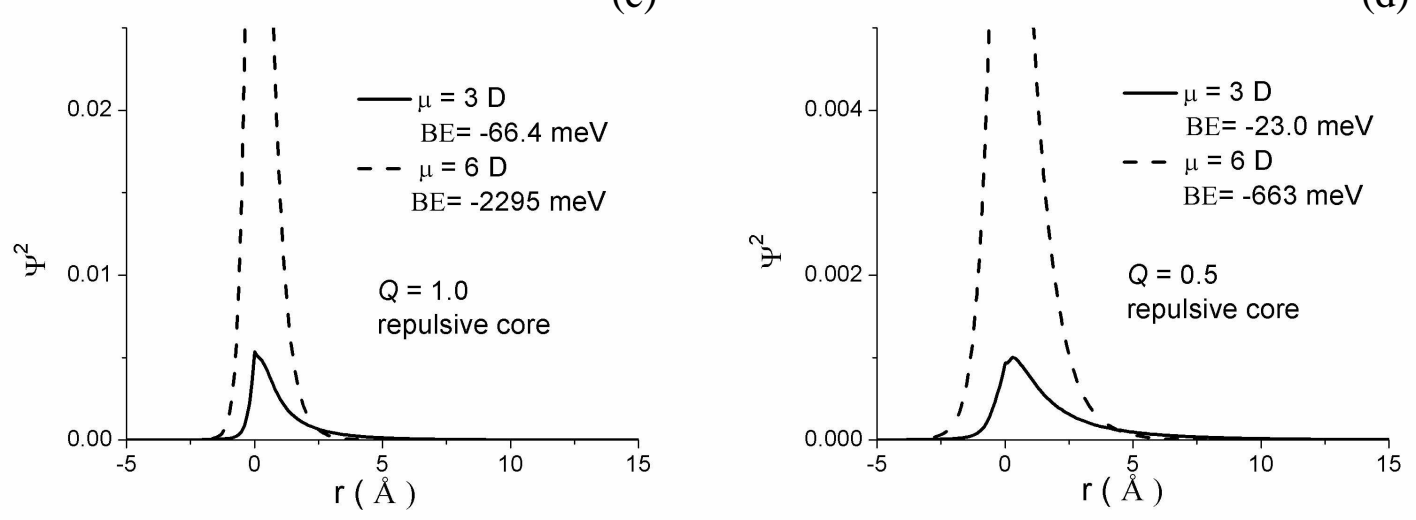
Figure 3. Definition of $\theta_{\mathrm{A}}, \theta_{\mathrm{B}}, \varphi$ and $\mathrm{r}$ used in Eq. 10

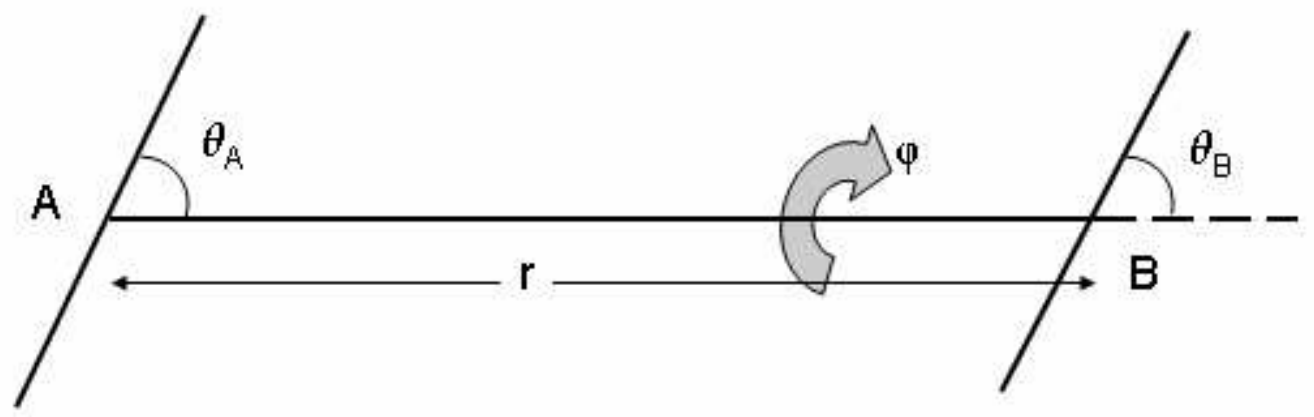


Figure 4. Drude model for describing the interaction between an excess electron and a neutral molecule with a permanent dipole described by two charges $+Q$ and $-Q$, separated by a distance $\left|\mathbf{r}_{1}-\mathbf{r}_{2}\right|$. Here the fixed $+q$ charge associated with the fictitious oscillator is located at the midpoint of the permanent dipole. The $-q$ charge associated with the Drude oscillator is separated from the $+q$ charge by $\mathbf{R}$.

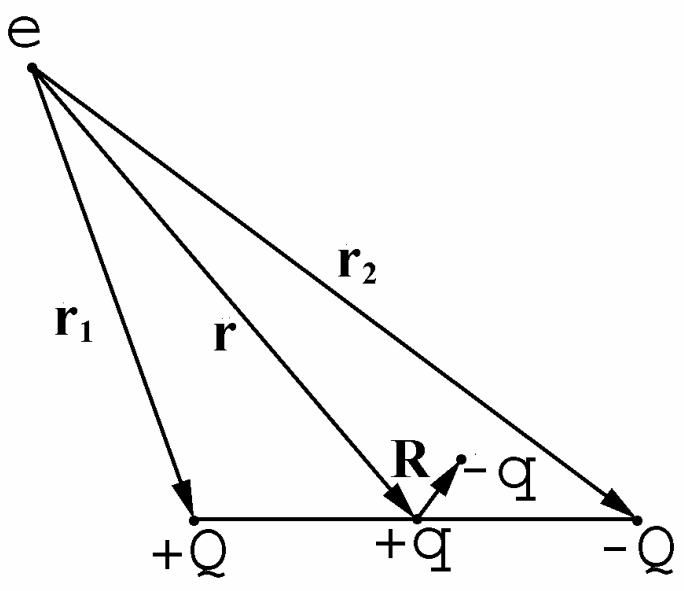


Figure 5. Treatment of polarization via the Drude model in the case of a molecular dimer, with the charge distribution of one monomer modeled by fixed point charges $Q_{l}^{(l)}$ and $Q_{2}^{(1)}=-Q_{1}{ }^{(1)}$ and that of the other modeled by fixed point charges $Q_{1}^{(2)}$ and $Q_{2}^{(2)}=-Q_{1}{ }^{(2)}$. The $+q$ and $-q$ charges are associated with the Drude oscillators.

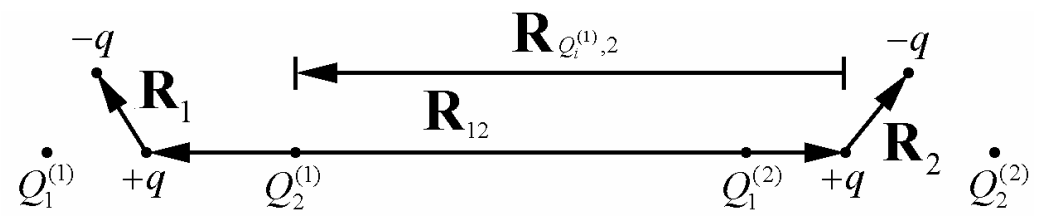


Figure 6. Definition of bond lengths used in Table I.

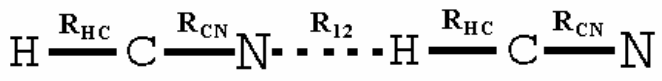

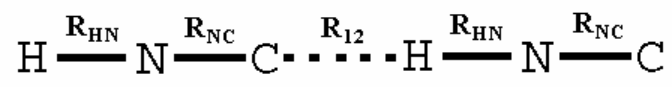


Figure 7. Comparison of the LUMO from the Hartree-Fock calculations and the most strongly bound electronic orbital from model I for HCN (a) and HNC (b).
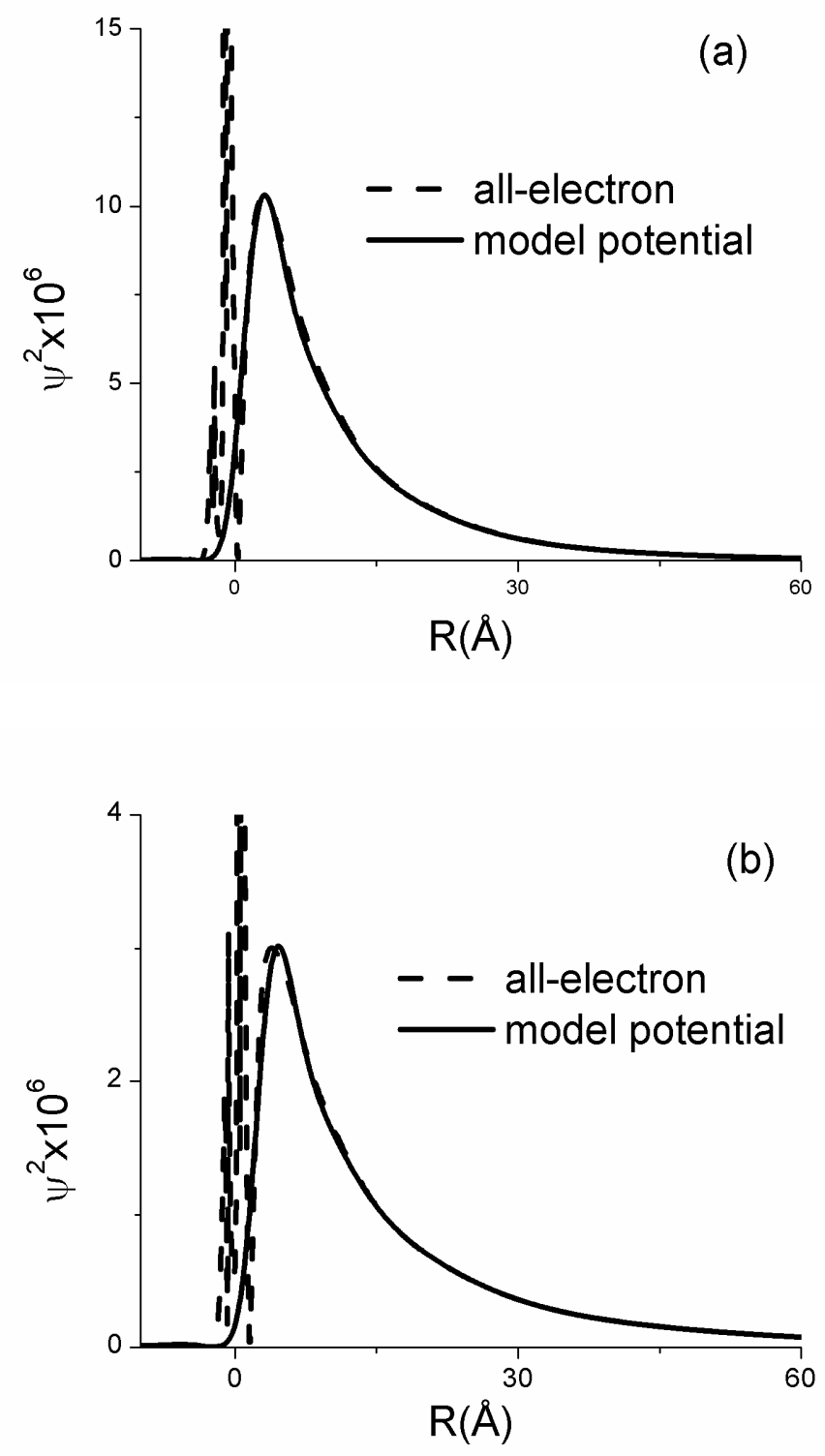
Figure 8. Time-of-flight mass spectrum of $\left(\mathrm{H}_{2} \mathrm{O}\right)_{n}{ }^{-}$from Ref. ${ }^{113}$. Note the magic numbers at $n=2,6,7$, and 11. Some of the minor peaks are due to mixed $\left(\mathrm{H}_{2} \mathrm{O}\right)_{n}{ }^{-} \mathrm{Ar}_{m}$ clusters. In fact, the peaks with appear to correspond to $\left(\mathrm{H}_{2} \mathrm{O}\right)_{4}{ }^{-}$and $\left(\mathrm{H}_{2} \mathrm{O}\right)_{8}{ }^{-}$are derive almost entirely from $\left(\mathrm{H}_{2} \mathrm{O}\right)_{2}{ }_{2} \mathrm{Ar}$ and $\left(\mathrm{H}_{2} \mathrm{O}\right)_{6}{ }^{-} \mathrm{Ar}$, respectively

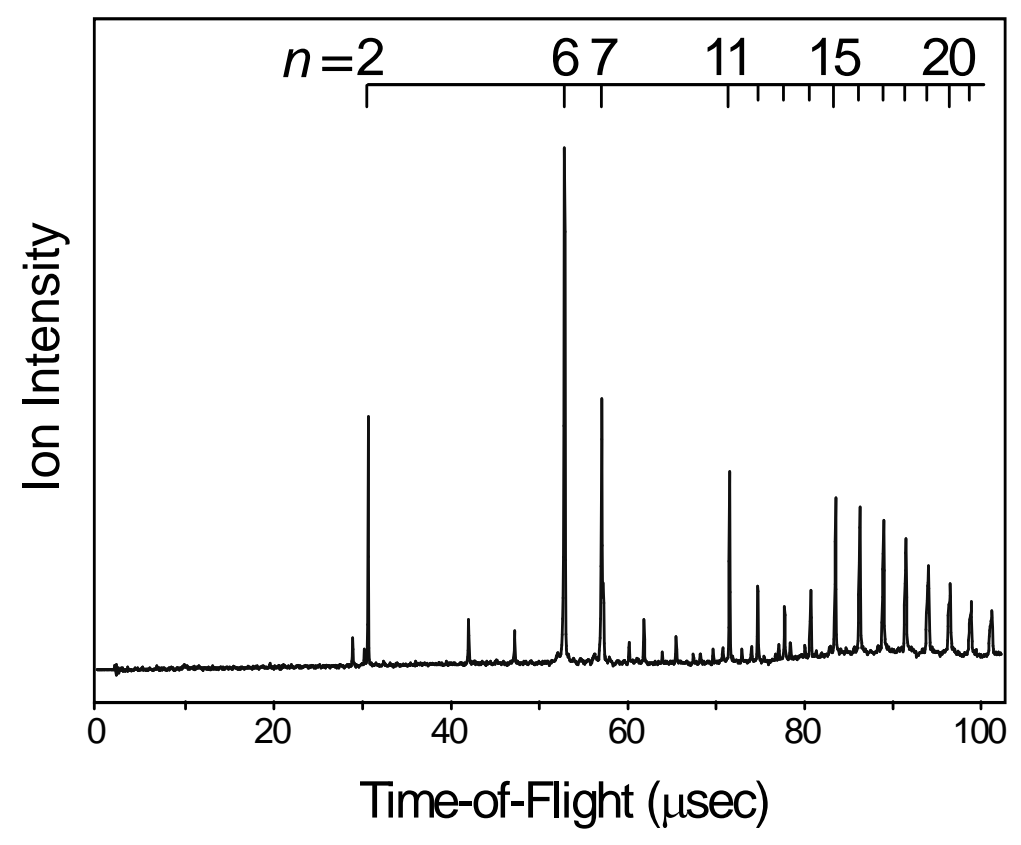


Figure 9. MP2 optimized geometries of the $\left(\mathrm{H}_{2} \mathrm{O}\right)_{n}{ }^{-}, \mathrm{n}=2-4$ clusters: $(\mathrm{a} 1)\left(\mathrm{H}_{2} \mathrm{O}\right)_{2}{ }^{-}$, Crown (b1) , transition state (b2), and chain (b3) structures of $\left(\mathrm{H}_{2} \mathrm{O}\right)_{3}{ }^{-}$, crown (c1) and chain (c2) forms of $\left(\mathrm{H}_{2} \mathrm{O}\right)_{4}$. For the crown and transition state structures, the small black dot indicates the center of the extended set of diffuse basis functions.

(a1)

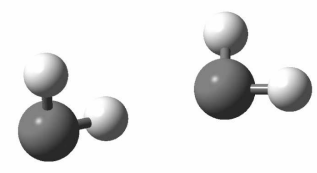

(b2)

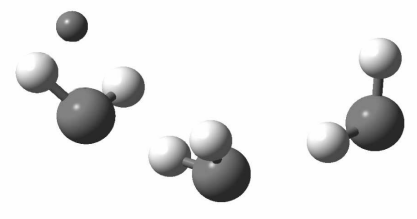

(c1)

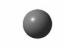

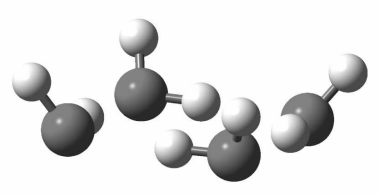

(b1)

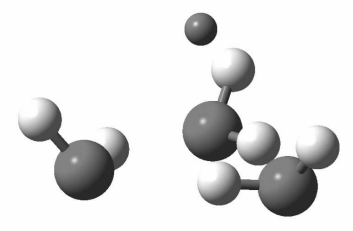

(b3)

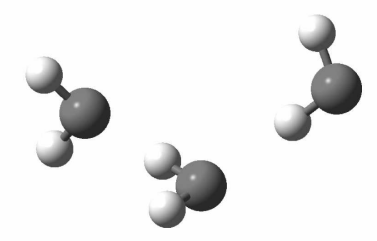

(c2)

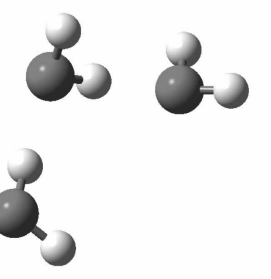


Fig 10. Plot of the electron density of the crown-like form of $\left(\mathrm{H}_{2} \mathrm{O}\right)_{4}{ }^{-}$calculated using the CI (solid line) and MP2 (dashed line) methods in conjunction with model II. The density is ploted along the $\mathrm{C}_{4}$ axis, with the origin being located in the plane of the four $\mathrm{O}$ atoms. (The free $\mathrm{OH}$ hydrogen atoms are located $0.75 \AA$ above the O-atom plane.)

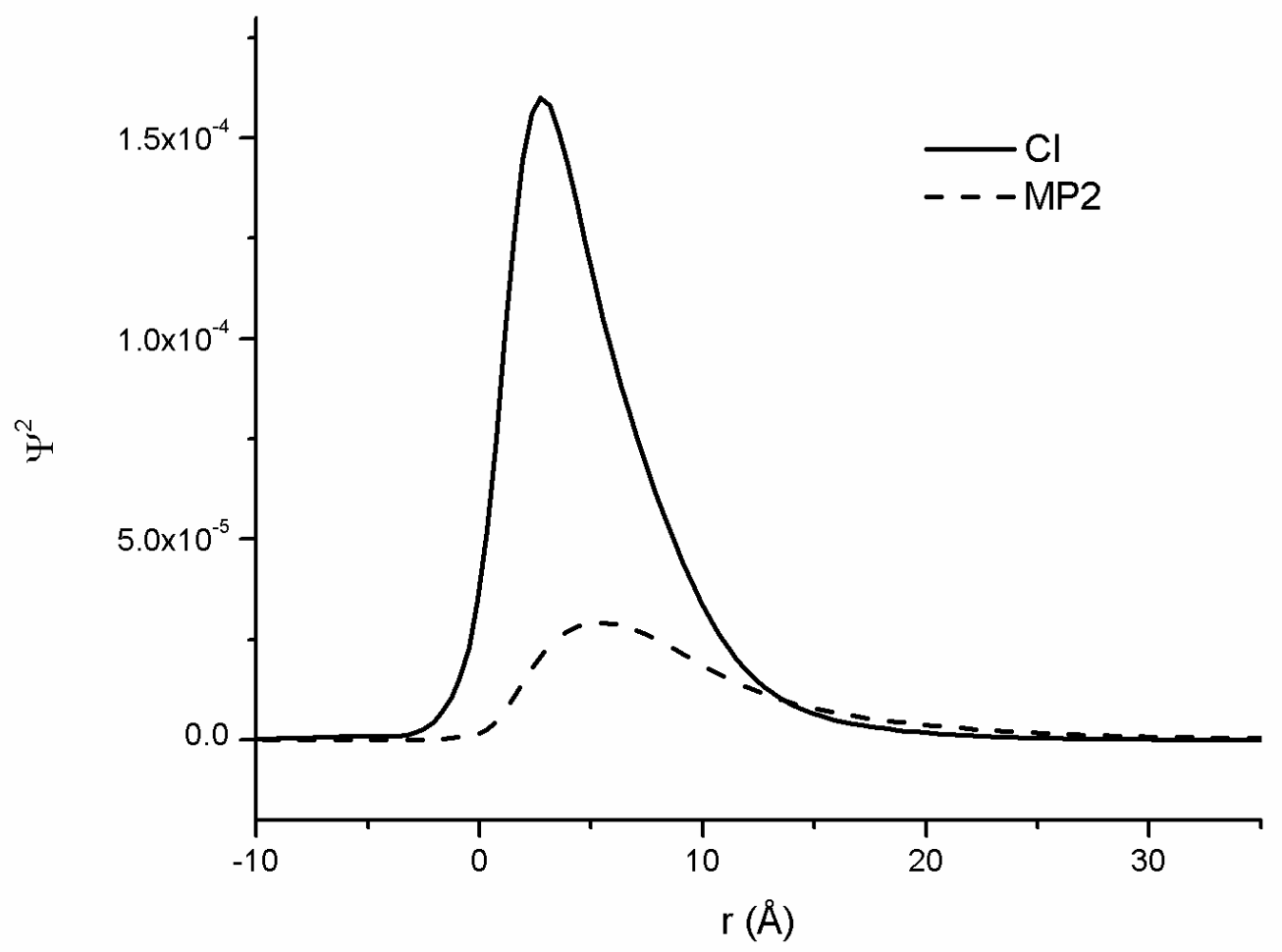


Figure 11. Plot of the electron density of the chain-like form of $\left(\mathrm{H}_{2} \mathrm{O}\right)_{4}{ }^{-}$calculated using the CI (solid line) and MP2 (dashed line) methods in conjunction with model II. The plot is along the direction of the dipole of the water chain. The origin is taken to coincide with the $\mathrm{O}$ atom of the terminal, acceptor water molecule.

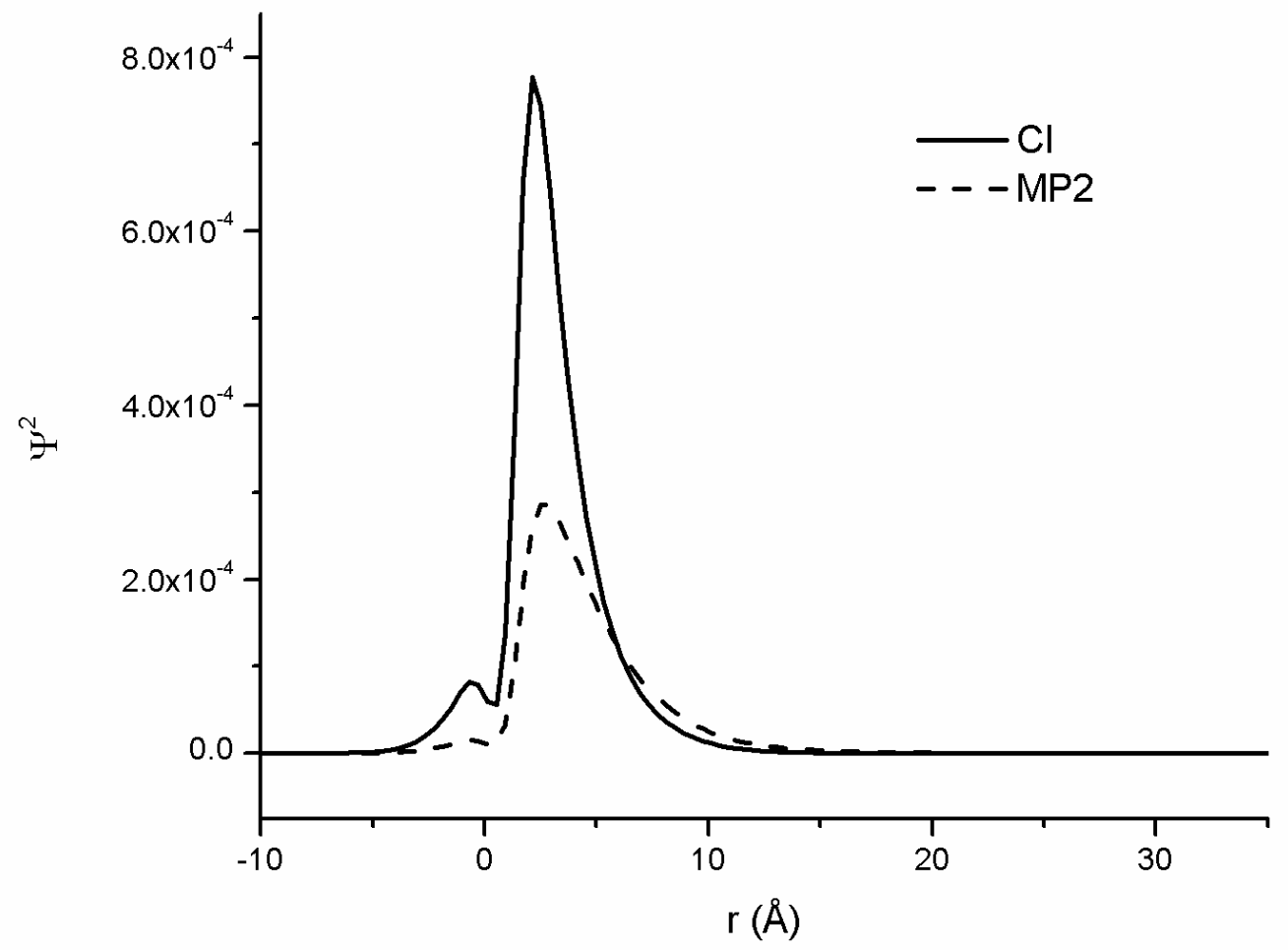


Figure 12. Plot of the electron density of the crown-like form of $\left(\mathrm{H}_{2} \mathrm{O}\right)_{4}{ }^{-}$calculated using the CI (solid line) and MP2 (dashed line) methods in conjunction with model II and model III, respectively. Model III is identical to model II except that the charges have been adjusted to reproduce the binding energy obtained from the model II CI calculations. The density is plotted along the $\mathrm{C}_{4}$ axis, with the origin being located in the plane of the four $\mathrm{O}$ atoms.

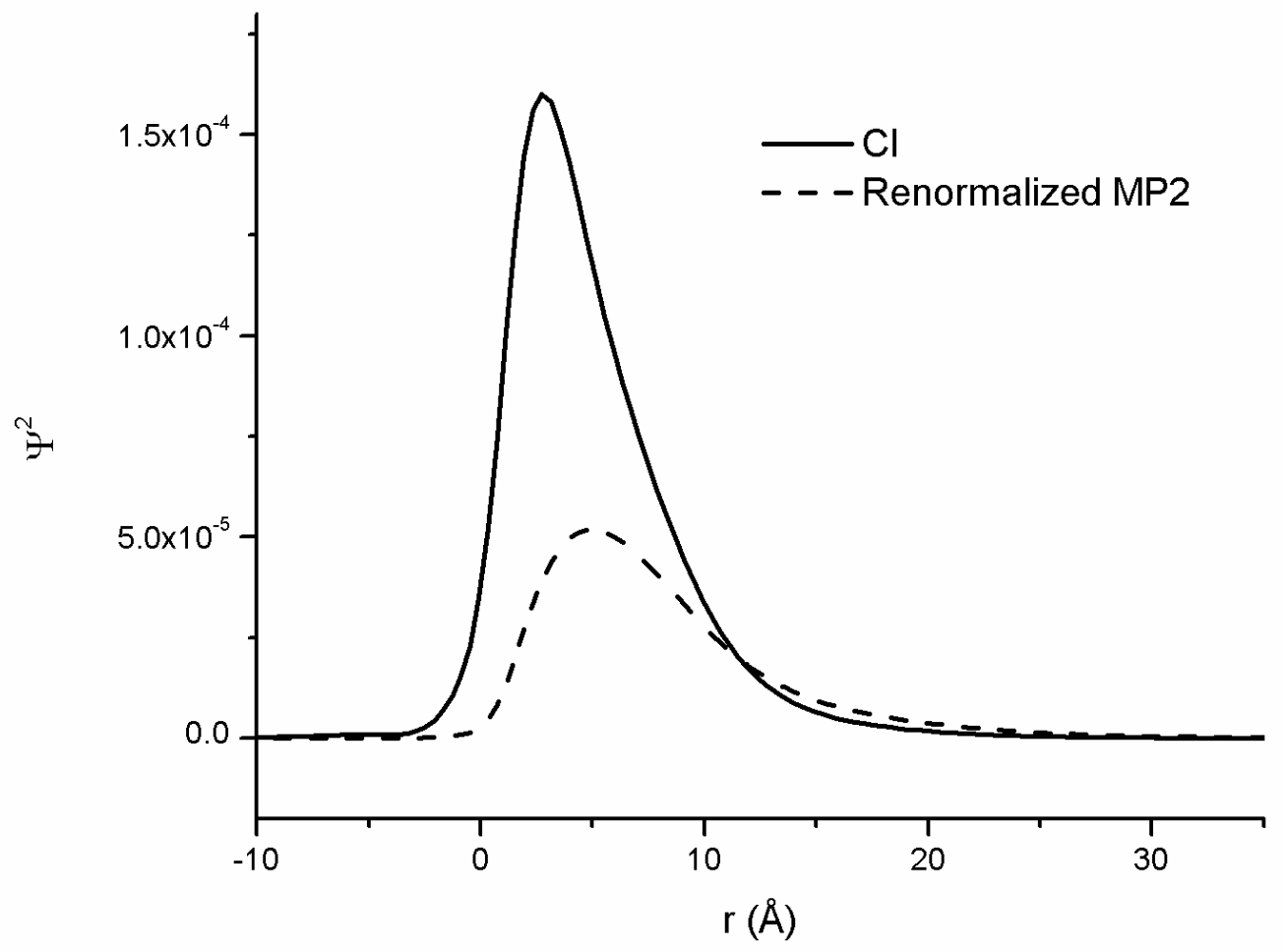


Figure 13. Plot of the electron density of the chain-like form of $\left(\mathrm{H}_{2} \mathrm{O}\right)_{4}{ }^{-}$calculated using the CI (solid line) and MP2 (dashed line) methods in conjunction with model II and model III, respectively. Model III is identical to model II except hat the charges have been adjusted to reproduce the binding energy obtained from the model II CI calculations. The plot is along the direction of the dipole of the water chain. The origin is taken to coincide with the $\mathrm{O}$ atom of the terminal, acceptor water molecule.

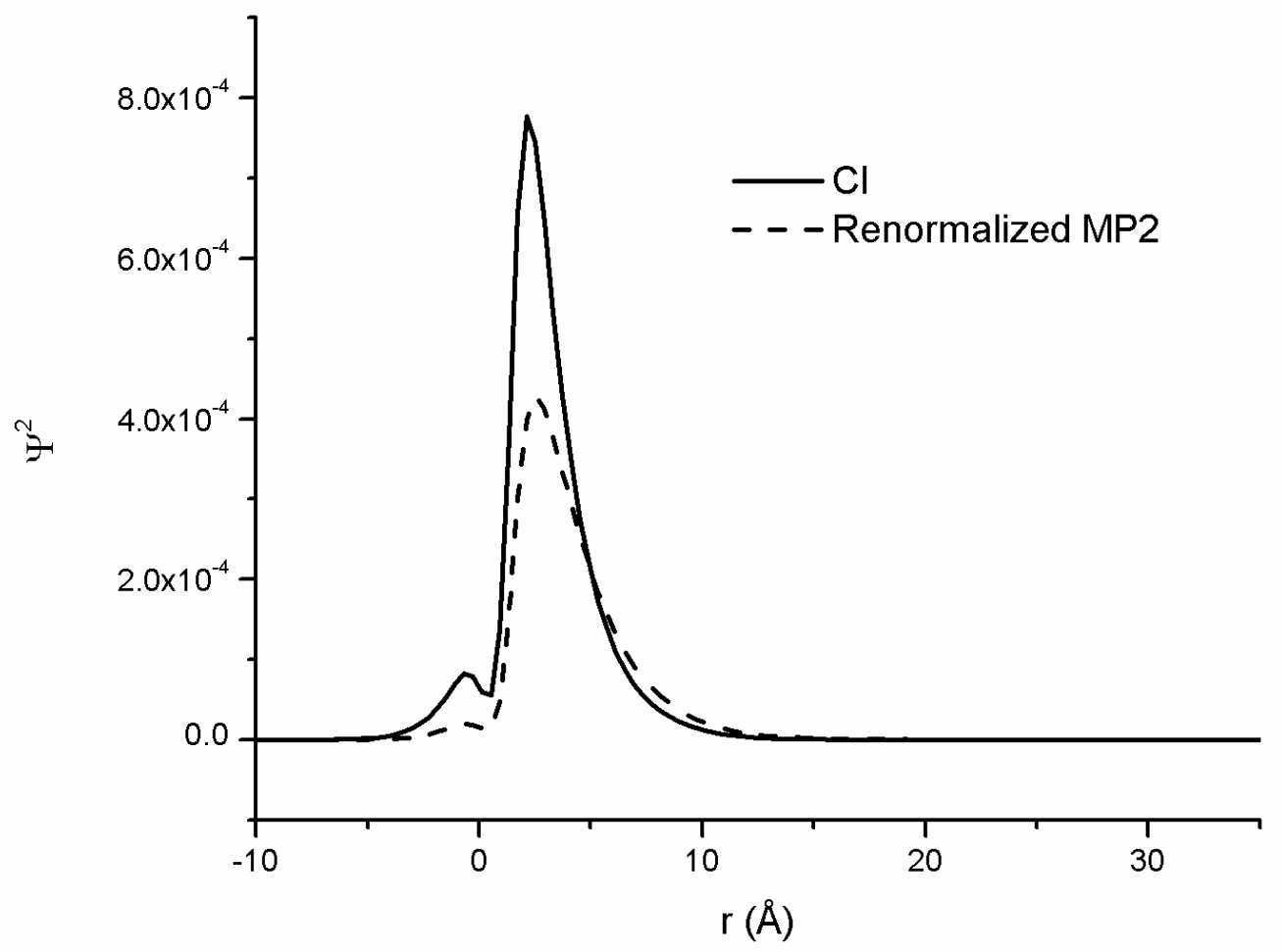


Figure 14. Representative configurations of $\left(\mathrm{H}_{2} \mathrm{O}\right)_{6}{ }^{-}$sampled in the 157 and $190 \mathrm{~K}$ replicas. Note that these structures are not at the local potential energy minima.

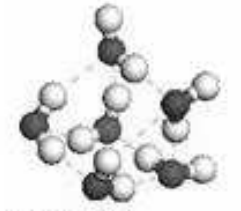

a) prism $\mu=6.187 \mathrm{D}$

$\mathrm{J}_{2}=6.20 \mathrm{au}$

$\mathrm{Et}=-47.55 \mathrm{kcal} / \mathrm{mol}$

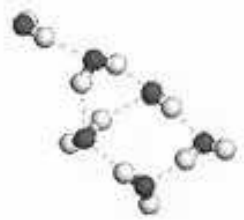

e) fused ring + tail $\mu=7.375 \mathrm{D}$

$\mathrm{J}_{\mathrm{Z}}=12.16 \mathrm{au}$

$\mathrm{Et}=-34.82 \mathrm{kcal} / \mathrm{mol}$

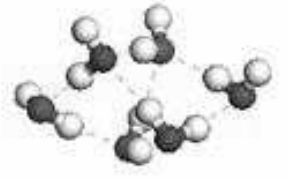

b) cage $\mu=6.879 \mathrm{D}$ $\mathrm{J}_{z}=7.98 \mathrm{au}$

$\mathrm{E} t=-46.35 \mathrm{kcal} / \mathrm{mol} \mathrm{Et}=-36.59 \mathrm{kcal} / \mathrm{mol}$

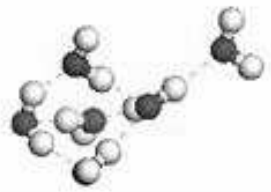

d) cage + tail $\mu=6.511 \mathrm{D}$ $\mathrm{J}_{z}=9.44 \mathrm{au}$

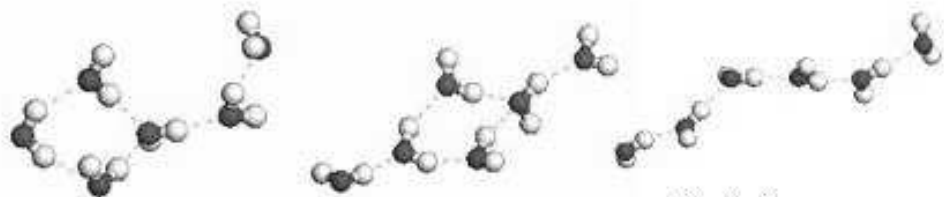

f) ring + tail $\mu=9.946 \mathrm{D}$ $\mathrm{J}_{z}=12.99 \mathrm{au}$

g) ring + tails

h) chain $\mu=12.451 \mathrm{D}$ $\mu=11.14 \mathrm{D}$ $\mathrm{J}_{z}=20.52 \mathrm{au}$ $\mathrm{Et}=-34.91 \mathrm{kcal} / \mathrm{mol} \quad \mathrm{Et}=-35.42 \mathrm{kcal} / \mathrm{mol}$

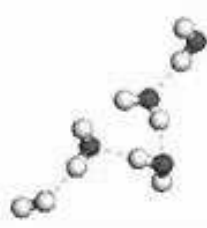

i) chain $\mu=11.07 \mathrm{D}$ $\mathrm{J}_{\mathrm{z}}=16.37 \mathrm{au}$ $\mathrm{E} t=-33.12 \mathrm{kcal} / \mathrm{mol}$

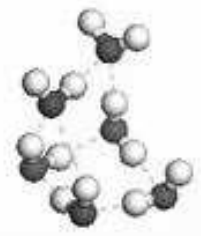

j) $\mathrm{dbs} 4 \mathrm{dbs} 2$ $\mu=9.542 \mathrm{D}$

$\mathrm{J} z=7.51$ au $\mathrm{Et}=-43.25 \mathrm{kcal} / \mathrm{mol}$

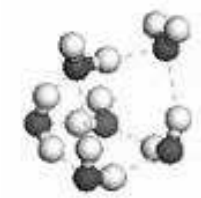

k) $\mathrm{db} s 4^{\prime} \mathrm{db} 2$ $\mu=8.479 \mathrm{D}$ $\mathrm{J}_{2}=6.96 \mathrm{au}$ $\mathrm{Et}=-40.87 \mathrm{kcal} / \mathrm{mol}$ 
Figure 15. Distribution of $\left(\mathrm{H}_{2} \mathrm{O}\right)_{6}$ structures as a function of $J_{z}$. (a) Number of configurations as a function of $J_{z}$, (b) Integration of (a) normalized to 1.0
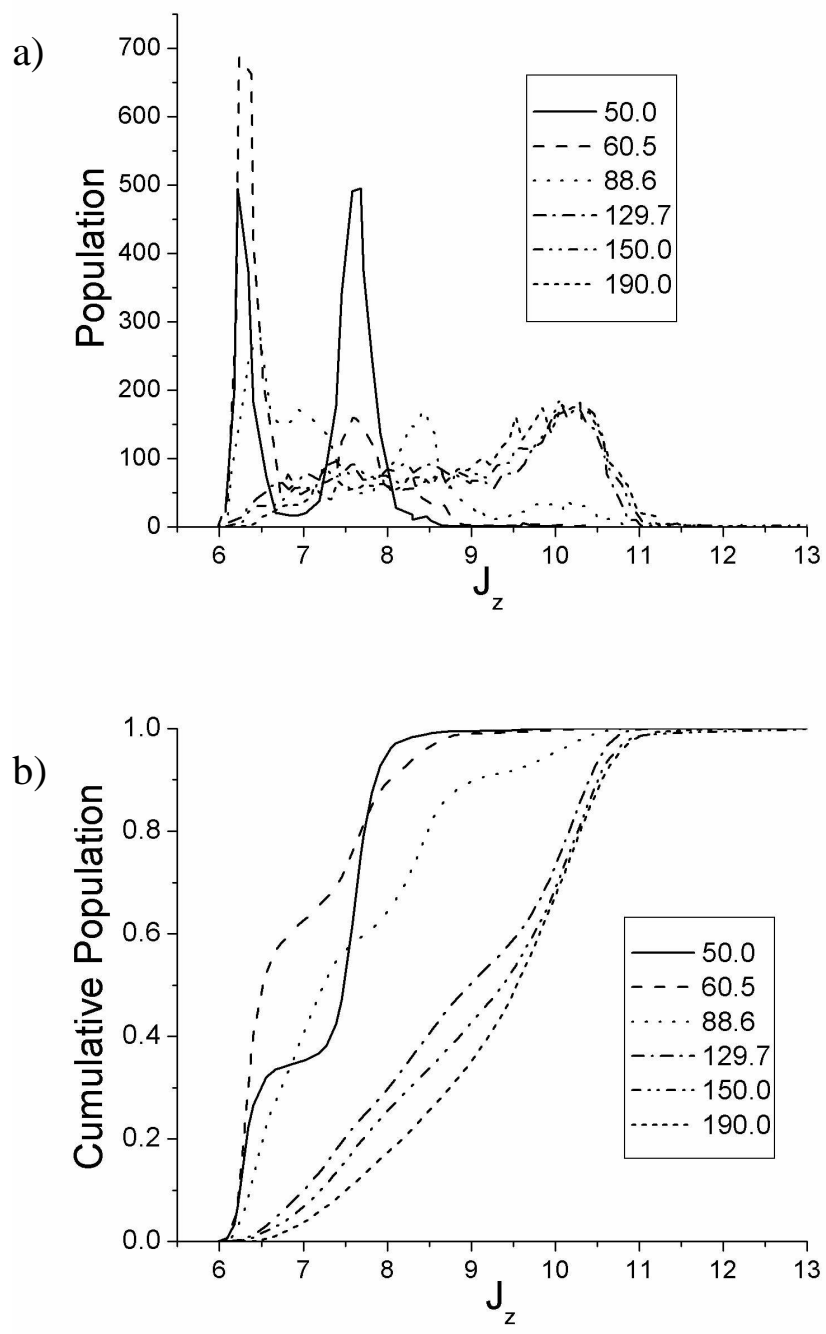
Figure 16. Distribution of $\left(\mathrm{H}_{2} \mathrm{O}\right)_{6}{ }_{6}^{-}$structures as a function of $J_{z}$. (a) Full histogram, (b) $5.5<J_{z}<10.0$, (c) integration of (a) with y axis normalized to 1.0
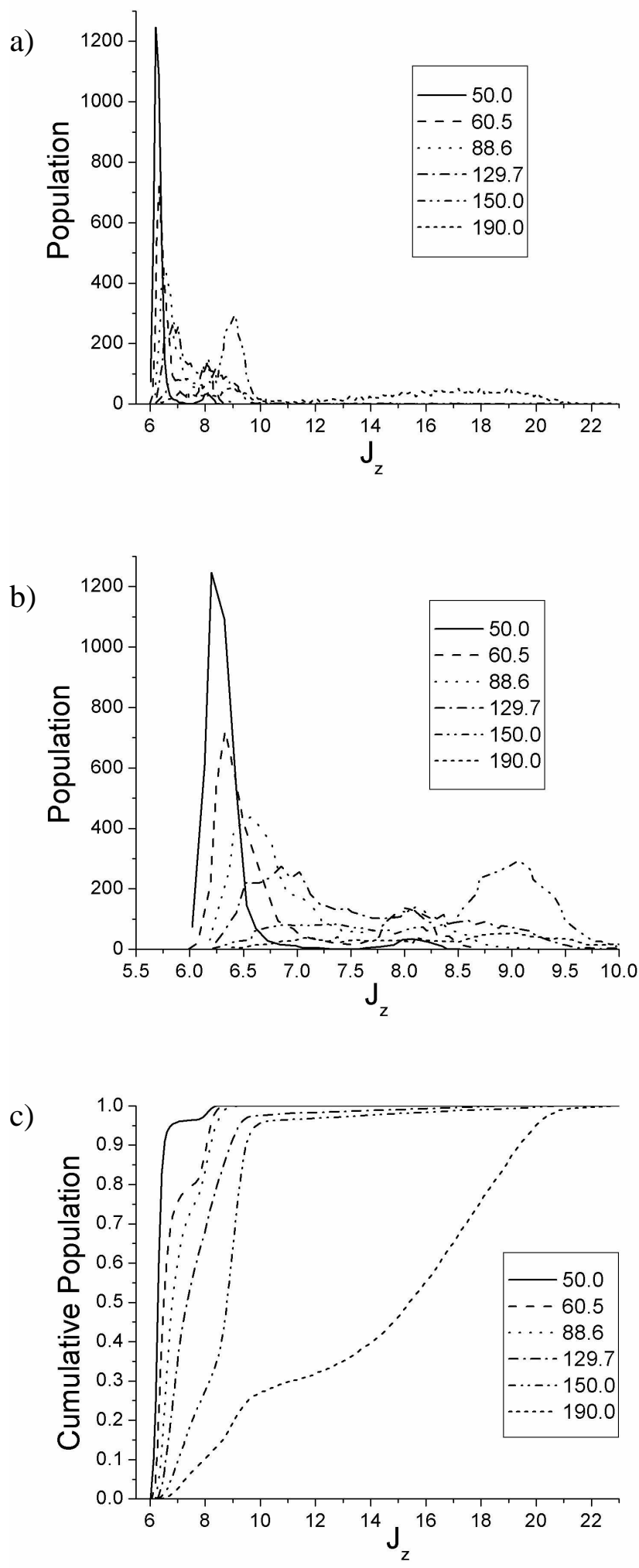
Figure 17. Selected local minima of $\left(\mathrm{H}_{2} \mathrm{O}\right)_{6}{ }^{-}$as described by the Drude model. The geometries were optimized using the smaller basis set for the excess electron, followed by single-point calculations of the total energies using the larger basis set.
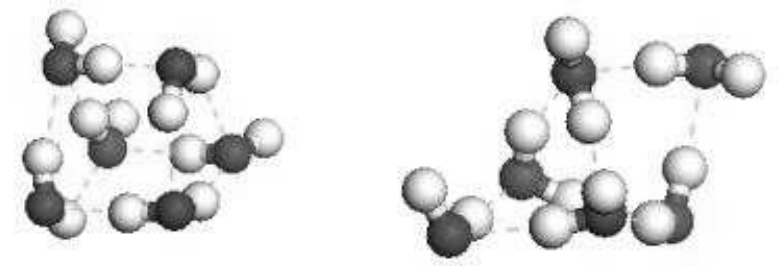
a) prism $\mu=6.971 \mathrm{D}$ $\mathrm{J}_{z}=6.12 \mathrm{au}$ $\mathrm{Et}=-48.90 \mathrm{kcal} / \mathrm{mol}$

b) cage $\mu=7.293 \mathrm{D}$

$\mathrm{J} z=7.81 \mathrm{au}$ $\mathrm{Et}=-48.32 \mathrm{kcal} / \mathrm{mol}$
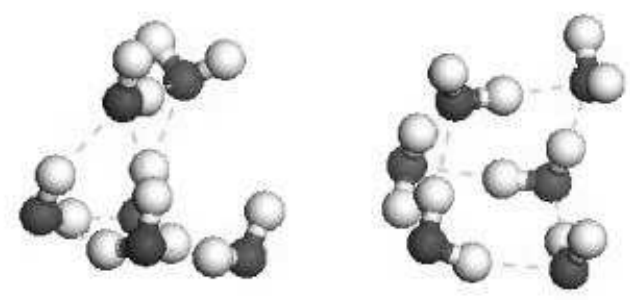

c) $d b s 4 d b s 2$
$\mu=9.466 \mathrm{D}$
$J_{z}=6.42 \mathrm{au}$
$E_{t}=-48.16 \mathrm{kcal} / \mathrm{mol}$

d) dbs4' dbs2 $\mu=9.253 \mathrm{D}$

$\mathrm{J}_{z}=6.58 \mathrm{au}$

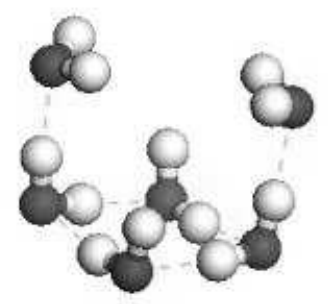

$\mathrm{E} t=-46.92 \mathrm{kcal} / \mathrm{mol}$

$$
\begin{aligned}
& \text { e) tweezers } \\
& \mu=7.938 \mathrm{D} \\
& J_{z}=7.85 \mathrm{au} \\
& \mathrm{E} t=-42.86 \mathrm{kcal} / \mathrm{mol}
\end{aligned}
$$


Figure 18. Arrangements of $(\mathrm{HF})_{n}, n=2-4$, with overall net dipole moments of zero.

(a) $n=2$, (b) $n=3$, (c) $n=4$.

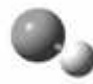

(a)
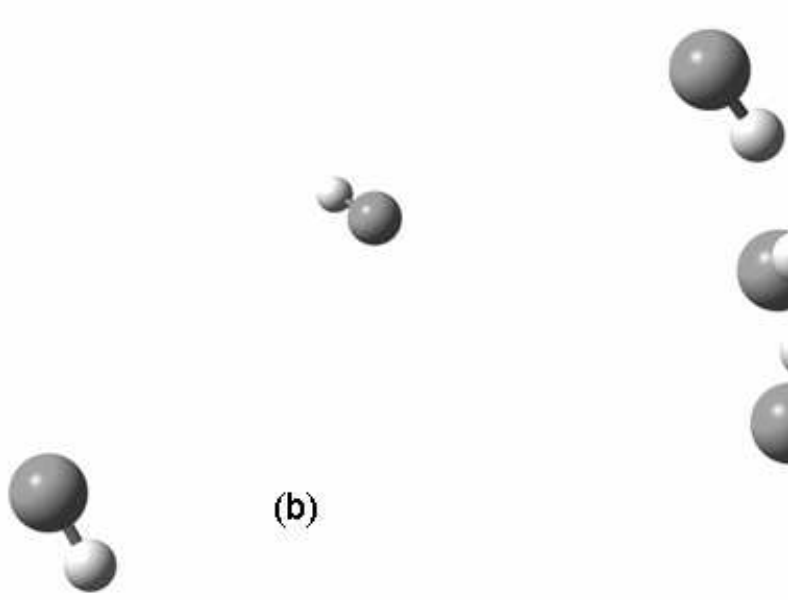

(c)

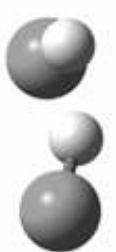

(b)

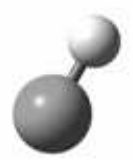


Figure 19. Schematic charge arrangements that can give bi-dipole-bound anion (a) and quadrupole bound anion (b) and (c)

(a)

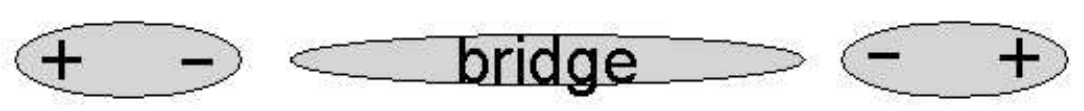

(c)

(b)

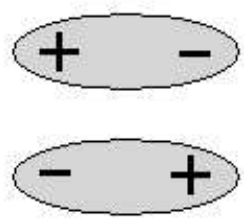

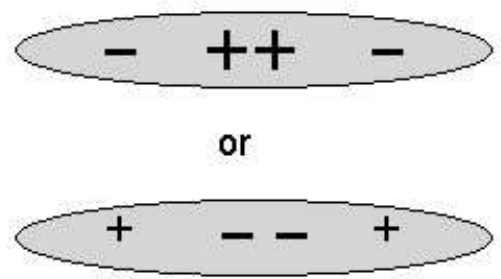




\title{
Appendix: ${ }^{1}$ \\ Theoretical Calculations of Voltage-dependent STM Images of Acetylene on the Si(001) Surface
}

\begin{abstract}
Voltage-dependent STM images have been calculated for five chemisorbed forms of acetylene on the $\mathrm{Si}(001)$ surface. The calculated images are used to aid in assigning the species observed in recent scanning tunneling microscope (STM) measurements on the $\mathrm{Si}(001) /$ acetylene system.
\end{abstract}

${ }^{1}$ Published as: F. Wang, D. C. Sorescu, and K. D. Jordan, J. Phys. Chem. B 106 (6), 1316 (2002). 


\section{INTRODUCTION}

The reactions of hydrocarbons with silicon surfaces are of considerable interest as routes to forming silicon carbide thin films and hybrid semiconductor/hydrocarbon based materials. $^{1,2}$ The reactions of ethylene and acetylene with the Si(001) surface, in particular, have been the subject of numerous experimental and theoretical studies.

The $\mathrm{Si}(001) /$ acetylene system has proven surprisingly controversial. Until recently, it was believed that the dominant form of $\mathrm{C}_{2} \mathrm{H}_{2}$ on the $\mathrm{Si}(001)$ surface resulted from the addition of acetylene molecules across an Si-Si surface dimers. ${ }^{3-7}$ Some early studies concluded that this addition process was accomplished by cleavage of the

dimer. $^{3-5}$ However, it is now well established that the Si-Si dimer remains intact following the adsorption of the acetylene molecule. ${ }^{6,7}$ In the present work this is referred to as the di- $\sigma$ species. Recent STM measurements of Mezhenny et al. ${ }^{8}$ have revealed that the $\mathrm{Si}(001) /$ acetylene system is more complicated than previously believed. In particular, these measurements provided evidence for three different binding sites for acetylene on the surface. These were attributed to the di- $\sigma$, "end-bridge", and "r-bridge" structures depicted in Fig. 1. (In the end-bridge structure the $\mathrm{C}_{2} \mathrm{H}_{2}$ molecule bridges the ends of two adjacent dimers in a dimer row, and in the r-bridge configuration the $\mathrm{C}_{2} \mathrm{H}_{2}$ molecule is oriented perpendicular to the dimer row and is tetra-coordinated to two dimers.) Mezhenny et al. also investigated these structures as well as a p-bridge tetra-coordinated structure with the $\mathrm{C}_{2} \mathrm{H}_{2}$ molecule parallel to the dimer row, using electronic structure theory. Although the p-bridge structure was predicted to be a local potential energy minimum, it was found to lie considerably higher in energy than the other three structures and was deemed less important. 
$\mathrm{Xu}$ et al., on the basis of photoelectron diffraction measurements, concluded that at high coverage the p-bridge structure is the dominant form of $\mathrm{C}_{2} \mathrm{H}_{2}$ on the $\mathrm{Si}(001)$ surface. ${ }^{9}$ On the other hand, Terborg et al., using the same technique, concluded that the dominant species is the di- $\sigma$ structure. ${ }^{10}$ Based on a comparison between calculated and measured vibrational spectra, Morikawa concluded that the di- $\sigma$ and end-bridge species co-exist on the surface. ${ }^{11}$ Morikawa did not find it necessary to invoke tetra-coordinated species in order to explain the vibrational spectra.

There have been two attempts to use calculations of the STM images of various $\mathrm{Si}(001) / \mathrm{C}_{2} \mathrm{H}_{2}$ species to assign the structure in the measured STM images. Mezhenny et $a l{ }^{8}$ used the Tersoff-Hamann approach ${ }^{12}$ together with cluster models of the adsorbed species to calculate STM images for comparison with their experimental results. Based on these calculations, it was concluded that there is a sizable population of both di- and tetra-coordinated $\mathrm{C}_{2} \mathrm{H}_{2}$ species on the $\mathrm{Si}(001)$ surface. The second such undertaking, by Hofer et al., ${ }^{13}$ employed a more sophisticated model of the STM tip and slab models with periodic boundary conditions. (We became aware of this latter work when in the final stages of preparing the present manuscript.) The calculated images of Hofer et al. also appear to be consistent with the interpretation that the observed STM images derive from both di- and tetra-coordinated $\mathrm{C}_{2} \mathrm{H}_{2}$, although the tetra-coordinated species were predicted to be much less stable.

Motivated by the results of Mezhenny et al., Sorescu and Jordan ${ }^{14}$ carried out a detailed density functional theory (DFT) study of the Si(001)/acetylene system, locating a total of nine different minima as well as the barriers between the low energy minima. The calculations predicted that at low coverage the di- $\sigma$ structure is most stable, followed 
by the end-bridge and r-bridge configurations, with the binding energies being -2.73 , -2.44 , and $-2.13 \mathrm{eV}$, respectively. (A negative binding energy implies a stable structure.) The p-bridge structure was predicted to be bound by only $-1.29 \mathrm{eV}$. The barriers for rearrangement from the r-bridge to the end-bridge and from the p-bridge to the di- $\sigma$ species were calculated to be $0.30 \mathrm{eV}$ and $0.82 \mathrm{eV}$, respectively. Based on these results, it appears unlikely that either the r-bridge or p-bridge structures would have appreciably population at room temperature.

The electronic structure calculations of Sorescu and Jordan also predicted that a structure with two acetylene molecules bonded to two adjacent Si-Si dimers in an endbridge manner (see Fig. 1) is of comparable stability to the end-bridge structure (i.e., that the binding energy per acetylene molecule is comparable in the two cases). Hereafter, this arrangement is designated as (end-bridge) $)_{2}$. This raises the possibility that the structure in the STM images which has been attributed to tetra-coordinated species in fact arises from the (end-bridge $)_{2}$ structure. To investigate this possibility we have undertaken new calculations of the STM images of five different $\operatorname{Si}(001) /$ acetylene species, including the (end-bridge $)_{2}$ species. These calculations are based on slab-models with periodic boundary conditions, and allow for the influence of the electric field between the tip and surface. The approach used is described briefly in Section II, followed by a presentation of the results.

\section{Calculations}

\section{i) Geometries}

The geometries of the di- $\sigma$, end-bridge, r-bridge, and p-bridge $\mathrm{Si}(001) / \mathrm{C}_{2} \mathrm{H}_{2}$ species were taken from the work of Sorescu and Jordan, ${ }^{14}$ who optimized the structures 
using plane-wave DFT with slab models and periodic boundary conditions. Specifically, we adopted the structures that these authors obtained using a supercell with eight $\mathrm{Si}-\mathrm{Si}$ surface dimers, in a $4 \times 4$ arrangement, and employing a total of five silicon layers. Although the (end-bridge) $)_{2}$ species was also considered by Sorescu and Jordan, the calculations on this species employed a smaller supercell and were for saturation coverage. For this reason, we have optimized the geometry of the (end-bridge) ${ }_{2}$ structure using the supercell with eight surface SiSi dimers. Only a single (end-bridge) $)_{2}$ species is present in the supercell. Following Sorescu and Jordan, the bottommost silicon layer was terminated by $\mathrm{H}$ atoms, and the terminating $\mathrm{H}$ atoms and the $\mathrm{Si}$ atoms in the bottom two layers were kept frozen in the geometry optimizations (the latter in their bulk positions). A vacuum layer of about $8 \AA$ separated the top of one slab and the bottom of the next. The Perdew-Wang91 (PW91) gradient-corrected exchange-correlation functional ${ }^{15}$ was employed together with ultrasoft pseudopotentials, ${ }^{16,17}$ a plane-wave cut-off of $200 \mathrm{eV}$, and $\Gamma$-point sampling. These calculations were performed with the VASP program. ${ }^{17-19}$

\section{ii) Calculation of tunneling currents.}

Most calculations of STM images have been carried out using the approach of Tersoff and Hamann, ${ }^{12}$ which employs the local density of states method, assumes a simple model ( $s$-level only) of the tip, and ignores electric field effects. This was the approach used in the above-mentioned calculations of Mezhenny et al. Hofer et al. used a similar approach but adopted a more realistic (atomistic) model of the tip. Both of these studies neglected field effects which can be important for modeling STM images of molecules on semiconductor surfaces since the experimental studies are often carried out with biases between 1.5 and 2 Volts. In fact, it has been demonstrated that the neglect of 
electric field effects leads to qualitatively incorrect images for the $\mathrm{Si}(001) /$ ethylene system. $^{20}$

In the present study, we employ a high-voltage extension of the Tersoff-Hamann approach due to Stockbro ${ }^{21}$ to calculate the STM images of the di- $\sigma$, end-bridge, r-bridge, p-bridge, and (end-bridge) $)_{2}$ forms of $\mathrm{Si}(001) / \mathrm{C}_{2} \mathrm{H}_{2}$. In this approach, the electric field between the tip and surface is accounted for self consistently, and higher spherical harmonics (i.e., $1>0$ ) can be used in the model of the tip. As for the geometry optimizations, the STM calculations were carried out using the PW91 exchangecorrelation functional, ${ }^{15}$ ultra-soft Vanderbilt pseudopotentials, ${ }^{16}$ and plane-wave basis sets. These calculations were performed using a modified version of the PWSCF code. $^{21,22}$

The calculations of the tunneling currents required several modifications of the procedure used for the geometry optimizations. First, the terminating $\mathrm{H}$ atoms were removed, and two additional layers of $\mathrm{Si}$ atoms at their bulk positions were added. (The presence of the dangling bonds on the bottom layer has the effect of introducing free carriers, which is essential for calculating the tunneling currents. ${ }^{21}$ ) The bias voltage between the tip and the sample was simulated by putting a dipole layer in the middle of the vacuum layer, which was chosen to be $10 \AA \AA$. (The use of a larger vacuum layer for the STM calculations than for the geometry optimization was necessary to permit reliable interpretation of the wavefunction in the region around which the tip is moving.) A planewave cutoff of $272 \mathrm{eV}$ and $\Gamma$ point sampling were used.

Self-consistent electronic structure calculations were done at bias voltages of 0.68 1.36, and $2.04 \mathrm{~V}$. The wave functions at the sample bias $(-1.5 \mathrm{~V})$ used in the experiments 
were obtained by logarithmic interpolation of the results of the calculations at the 0.68 , 1.32, and 2.04 V bias. The surface geometry was not relaxed in the external field as test calculations showed that such relaxation was relatively unimportant for the tunneling current.

The tail of the wavefunction far from the sample surface is difficult to calculate in conventional DFT treatments. The modified PWSCF code deals with this problem by integrating the wavefunction tails using an average effective potential method of Tersoff. $^{23}$ This integration procedure was adopted for distances greater than $3.2 \AA$ from the silicon surface. Calculations were carried out using both $s$, and $s$ plus $p$ models of the tip. These gave very similar images, and the results reported here were obtained with the $s$-type tip.

\section{Results and Discussion}

Figure 2 reproduces the measured STM image of Mezhenny et al., and Figures 38 give our calculated images for the bare $\mathrm{Si}(001) /(2 \times 1)$ surface and the five $\mathrm{Si}(001) / \mathrm{C}_{2} \mathrm{H}_{2}$ species described above. In comparing the measured and calculated images, it should be kept in mind that the measured image was obtained at room temperature, whereas the calculations implicitly assume a temperature of $0 \mathrm{~K}$. This is an important consideration in that the bare dimers on the $\mathrm{Si}(001)$ surface, while believed to be buckled at low temperatures, are expected to appear symmetric at room temperatures due to the lowbarriers for flipping ${ }^{24,25}$ (see however, Ref. 26). The buckling of the bare surface is readily apparent in the calculated STM-images (Fig. 3-8). Since buckling changes the appearance of the STM image, the use of $0 \mathrm{~K}$ calculations could introduce some ambiguitities in the comparison with experiment. However, we note that there is evidence 
of buckling in the observed STM images of the bare dimer regions of the $\mathrm{Si}(001) /$ acetylene surface. This may be the result of "pinning" caused by defects and adsorbed acetylene molecules.

The calculations give a darker image at the di- $\sigma$ site (Fig. 3) than the surrounding bare surface, consistent with the observed image in the vicinity of what has been attributed to the di- $\sigma$ site. Similarly, the corrugation calculated for the end-bridge form of $\mathrm{Si}(001) / \mathrm{C}_{2} \mathrm{H}_{2}$ closely matches that of region II in the measured spectrum and which was attributed by Mezhenny et al. to an end-bridge site. The two tetra-coordinated structures give similar images, in agreement with the earlier STM calculations. ${ }^{8,13}$ The calculated images for the tetra-coordinated $\mathrm{C}_{2} \mathrm{H}_{2}$ species qualitatively resemble that of region III in the measured STM spectrum, which was attributed by Mezhenney et al. and by Hofer et al. to a tetra-coordinated $\mathrm{C}_{2} \mathrm{H}_{2}$ entity. However, our calculated images for the tetra-coordinated sites are not as dark compared to the bare surface as is region III in the experimental STM image.

Figure 8 shows the calculated STM image for the (end-bridge) $)_{2}$ species with two $\mathrm{C}_{2} \mathrm{H}_{2}$ molecules bound in an end-bridge manner to a pair of adjacent $\mathrm{Si}$-Si dimers. This image is also in good agreement with that associated with region III of the experimental image, lending support to our proposal that the observed image is due to a site with two bound $\mathrm{C}_{2} \mathrm{H}_{2}$ molecules.

The calculations also predict that the (end-bridge $)_{2}$ species is energetically more stable than two separate end-bridge species. (The binding energy per acetylene molecule is calculated to be $-2.61 \mathrm{eV}$ in the (end-bridge) ${ }_{2}$ structure $v s .-2.44 \mathrm{eV}$ for the end-bridge structure. This leads naturally to the question why the measured STM images do not 
show evidence of extended domains of (end-bridge) $)_{2}$ species? Additional studies

examining the barriers for formation of isolated and neighboring (end-bridge) $)_{2}$ species could prove useful in answering this question.

\section{Conclusions}

STM images have been calculated for five forms of acetylene on the $\mathrm{Si}(001)$ surface. The calculations allow in a self-consistent manner for the influence of the electric field of the tip on the wavefunction of the surface. Based on the comparison of the calculated and measured STM images, we conclude, in agreement with Mezhenny et al. that there are two types of di-coordinated $\mathrm{C}_{2} \mathrm{H}_{2}$ (the so-called di- $\sigma$ and end-bridge species) on the $\mathrm{Si}(001)$ surface. Our calculations also predict that an (end-bridge) $)_{2}$ species with two $\mathrm{C}_{2} \mathrm{H}_{2}$ molecules bonded in a end-bridge manner to two adjacent $\mathrm{Si}-\mathrm{Si}$ dimers is energetically more stable than two well-separated end-bridge species on the surface. Although the calculated STM images alone do not allow us to distinguish between the (end-bridge) $)_{2}$ and a tetra-coordinate $\mathrm{C}_{2} \mathrm{H}_{2}$ as the source of the third structure observed in the STM experiments, based on the energetics, we propose that the third structure is due to the (end-bridge) ${ }_{2}$ species. The inability of the calculated STM images to differentiate between the different assignments of this structure could be due to the calculations being carried out for $\mathrm{T}=0 \mathrm{~K}$ whereas the experimental images were obtained at room temperature. Low-temperature STM measurements might thus prove useful for definitively establishing the identity of structure III observed in the experiments of Mezhenny et al. 


\section{Acknowledgements:}

This research was carried out with the support of the National Science

Foundation. We thank Prof. K. Stokbro for use of his computer program for calculating the STM images and for valuable discussions about its use. We thank Prof. J. Yates and. S. Mezhenny for sharing with us their unpublished STM data for the Si(001)/acetylene system. The calculations were performed on the IBM RS6000 43P computers in the University of Pittsburgh's Center for Molecular and Materials Simulations. These computers were funded by grants from the NSF and IBM. 


\section{References:}

1. Okumura, H; Sakuma, E; Lee, J. H.; Mukaida, H.; J. Appl. Phys. 1987, 61, 1134.

2. Waldrop, J. R.; Grant, R. W. Appl. Phys. Lett. 1993, 62, 2685.

3. Taylor, P. A.; Wallace, R. M.; Cheng, C. C.; Weinberg, W. H.; Dresser, M. J.; Choyke, W. J.; Yates, J. T., Jr. J. Am. Chem. Soc. 1992, 114, 6754.

4. Craig, B. I.; Smith, P. V. Surf. Sci. 1992, 276, 174.

5. Cramer, C. S.; Weiner, B.; Frenklach, M. J. Chem. Phys. 1993, 99, 1356.

6. Li, L.; Tindall, C.; Takaoka, O.; Hasegawa, Y.; Sakurai, T. Phys. Rev. B. 1997, 56, 4648.

7. Nishijima, M.; Yoshinobu, J.; Tsuda, H.; Onchi, M. Surf. Sci. 1987, 192, 383.

8. Mezhenny, S.; Lyubinetsky, I.; Choyke, W. J.; Wolkow, R. A.; and Yates, J. T. Jr. Chem. Phys. Lett. 2001, 344,7

9. Xu, S. H.; Keeffe, M.; Yang, Y.; Chen. C.; Yu, M.; Lapeyre, G. J.; Rotenberg, E.;

Denlinger, J.; Yates, J. T. Jr. Phys. Rev. Lett. 2000, 84, 939.

10. Terborg, R.; Baumgartel, P.; Lindsay, R.; Schaff, O.; Gieszel, T.; Hoeft, J. T., Polcik, M.; Toomes, R. L.; Kulkarni, S.; Bradshaw, A. M.; Woodruff, D. P. Phys. Rev. B. 2000, $61,16697$.

11. Morikawa, Y. Phys. Rev. B. 2001, 63, 33405.

12. Tersoff, J.; Hamann, D. R. Phys. Rev. B 1985, 31, 805.

13. Hofer, W. A.; Fisher, A. J.; Wolkow R. A. Surf. Sci. 2001, 175, 83.

14. Sorescu D. C.; Jordan K. D. J. Phys. Chem. B 2000, 104, 8259.

15. Perdew, J. P.; Chevary, J. A.; Vosko, S. H.; Jackson, K. A.; Pederson, M. R.; Singh, D. J.; Fiolhais C. Phys. Rev. B 1992, 46, 6671. 
16. Vanderbilt, D. Phys. Rev. B 1990, 41, 7892.

17. Kresse, G.; Hafner, J. Phys. Rev. B 1993, 48, 13115.

18. Kresse, G.; Furthmüller, J. Comput. Mater. Sci. 1996, 6, 15.

19. Kresse, G.; Furthmüller, J. Phys. Rev. B 1996, 54, 11169.

20. Ness, H.; Fisher, A. J.; Briggs, G. A. D. Surf. Sci. 1997, 380, L479.

21. Stokbro, K.; Quaade, U.; Grey, F. Appl. Phys. 1998, A 66, S907.

22. Baroni, S.; Dal Corso, A.; de Gironcoli, S.; Giannozzi, P.; http://www.pwscf .org

23. Tersoff, J. Phys. Rev. B 1989, 40, 11990.

24. Hamers, R. J.; Tromp, R. M.; Demuth, J. E. Phys. Rev. B 1986, 34, 5343.

25. Yokoyama, T. Phys. Rev. B 2000, 61 R5078.

26. Some researchers have questioned the existence of buckling on the bare defect free Si(001)-(2x1) surface at low temperature. See e.g. Shoemaker, J.; Burggraf, L. W.;

Gordon, M. S. J. Chem. Phys., 2000, 112, 2994. 


\section{Figure Captions:}

Fig. 1. Definitions of various $\mathrm{Si}(001) / \mathrm{C}_{2} \mathrm{H}_{2}$ species. A, di- $\sigma ; \mathrm{B}$, end-bridge; C, p-bridge; D, r-bridge; E, (end-bridge) 2 . The black dots refer to the positions of the Si atoms of the surface $\mathrm{Si}-\mathrm{Si}$ dimers, the light grey dots to the $\mathrm{C}$ atoms, and the darker grey dots to the $\mathrm{H}$ atoms.

Fig. 2. Measured STM image of the $\mathrm{Si}(001) / \mathrm{C}_{2} \mathrm{H}_{2}$ system at low coverage reproduced with permission from Ref. 8. (a) Shows the measured image, from which three types of $\mathrm{C}_{2} \mathrm{H}_{2}$ binding sites (I-III) are identified. (b) indicates the assignments proposed in Ref 8 . (c) depicts the measured corrugation in the vicinity of the various binding sites.

Fig. 3. (a) Calculated STM image (30 ̊ x $30 \AA)$ of the bare Si(001)-(2x1) surface with a $c(4 \times 2)$ buckling pattern. The X's mark the positions of the surface Si atoms. (b) reports the calculated corrugation for motion of the STM tip across the dimers perpendicular to the dimer rows. The trajectory of the tip is shown by the horizontal line in (a).

Fig. 4. (a) Calculated STM image (30 ̊ x $30 \AA$ ) of the bare Si(001)-(2x1) surface with acetylene molecules bound in di- $\sigma$ sites. The X's denote the surface Si atoms, and the bright boxes and dark dots denote the position of the $\mathrm{C}$ and $\mathrm{H}$ atoms, respectively. (b) Comparison of the calculated and measured corrugation for motion of the STM tip perpendicular to the dimer rows (as indicated by the horizontal line in (a)). The light line refers to experiment (Ref. 8) and the dark line to the present calculations.

Fig. 5. (a) Calculated STM image (30 ̊ x $30 \AA)$ of the bare $\operatorname{Si}(001)-(2 \times 1)$ surface with acetylene molecules bound in end-bridge sites. The X's denote the surface Si atoms, and the bright boxes and dark dots the position of the $\mathrm{C}$ and $\mathrm{H}$ atoms respectively. (b) Comparison of the calculated and measured corrugation for motion of the STM tip 
perpendicular to the dimer rows (as indicated by the horizontal line in (a)). The light line refers to experiment (Ref. 8) and the dark line to the present calculations.

Fig. 6. (a) Calculated STM image ( $30 \AA$ x $30 \AA$ ) of the bare $\mathrm{Si}(001)-(2 \times 1)$ surface with acetylene molecules bound in p-bridge sites. The X's denote the surface Si atoms, and the bright boxes and dark dots the position of the $\mathrm{C}$ and $\mathrm{H}$ atoms respectively. (b) Comparison of the corrugation calculated for motion of the STM tip perpendicular to the dimer rows for the p-bridge species (as indicated by the horizontal line in (a)) and that measured in Ref. 8. for site III (See Fig. 2). The calculated and measured results are designated by the dark and light lines, respectively.

Fig. 7. (a) Calculated STM image ( $30 \AA x 30 \AA)$ of the bare $\operatorname{Si}(001)-(2 \times 1)$ surface with acetylene molecules bound in r-bridge sites. The X's denote the surface $\mathrm{Si}$ atoms, and the bright boxes and dark dots the position of the $\mathrm{C}$ and $\mathrm{H}$ atoms respectively. (b) Comparison of the corrugation calculated for motion of the STM tip perpendicular to the dimer rows for the r-bridge species (as indicated by the horizontal line in (a)) and that measured in Ref. 8. for site III (See Fig. 2). The calculated and measured results are designated by the dark and light lines, respectively.

Fig. 8. (a) Calculated STM image ( $30 \AA$ x $30 \AA$ ) of the bare $\mathrm{Si}(001)-(2 \times 1)$ surface with acetylene molecules bound in (end-bridge) $)_{2}$ sites. The X's denote the surface Si atoms, and the bright boxes and dark dots the position of the $\mathrm{C}$ and $\mathrm{H}$ atoms respectively. (b) Comparison of the corrugation calculated for motion of the STM tip perpendicular to the dimer rows for the (end-bridge) $)_{2}$ species (as indicated by the horizontal line in (a)) and that measured in Ref. 8. for site III (See Fig. 2). The calculated and measured results are designated by the dark and light lines, respectively. 


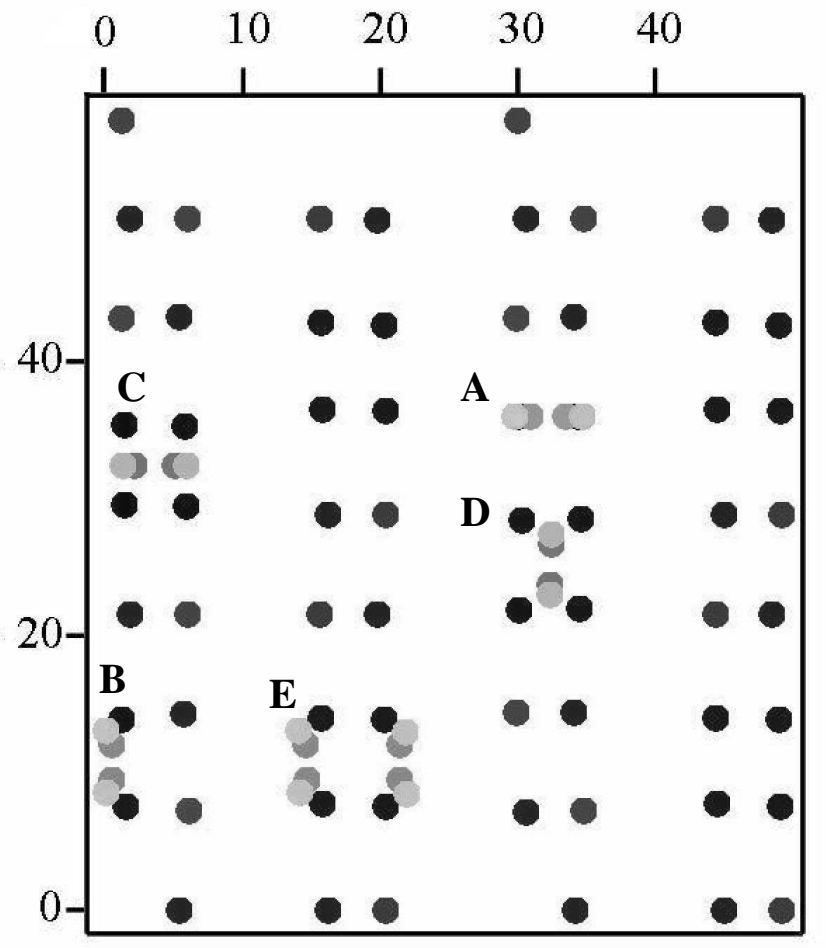



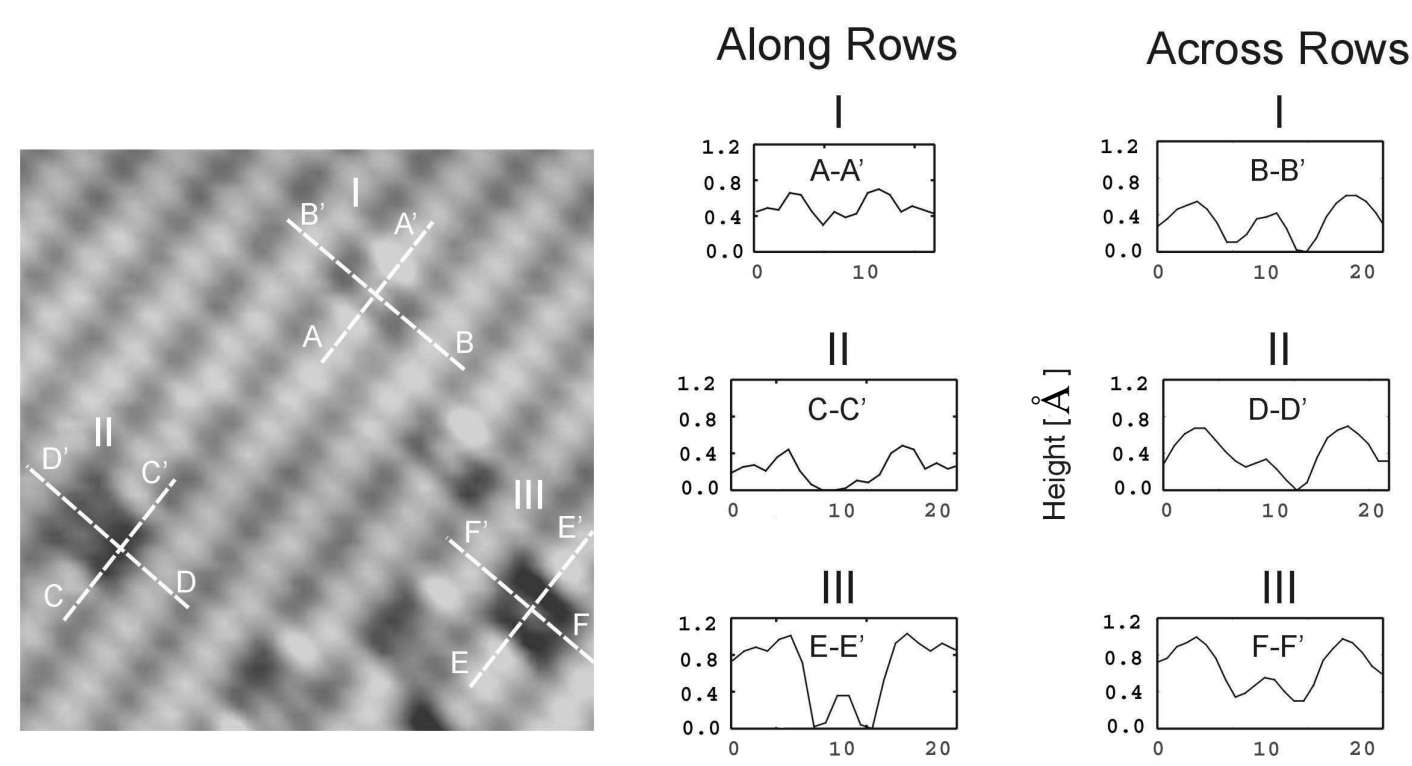

(a)

Distance [Å]

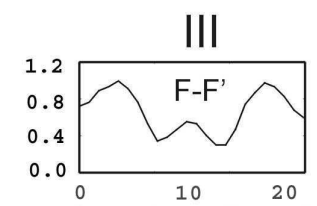

(C)

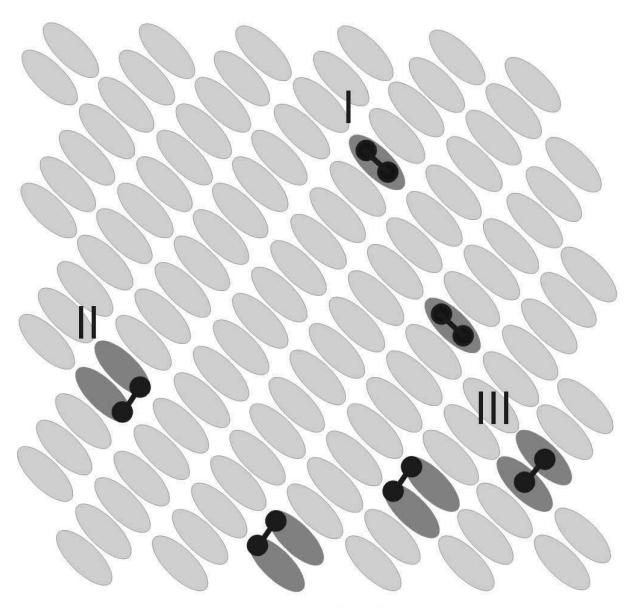

(b)

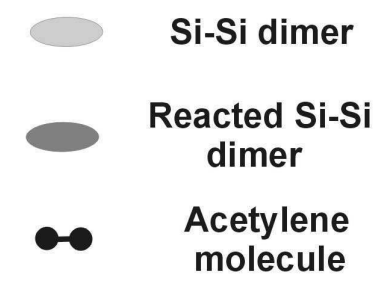


(a)

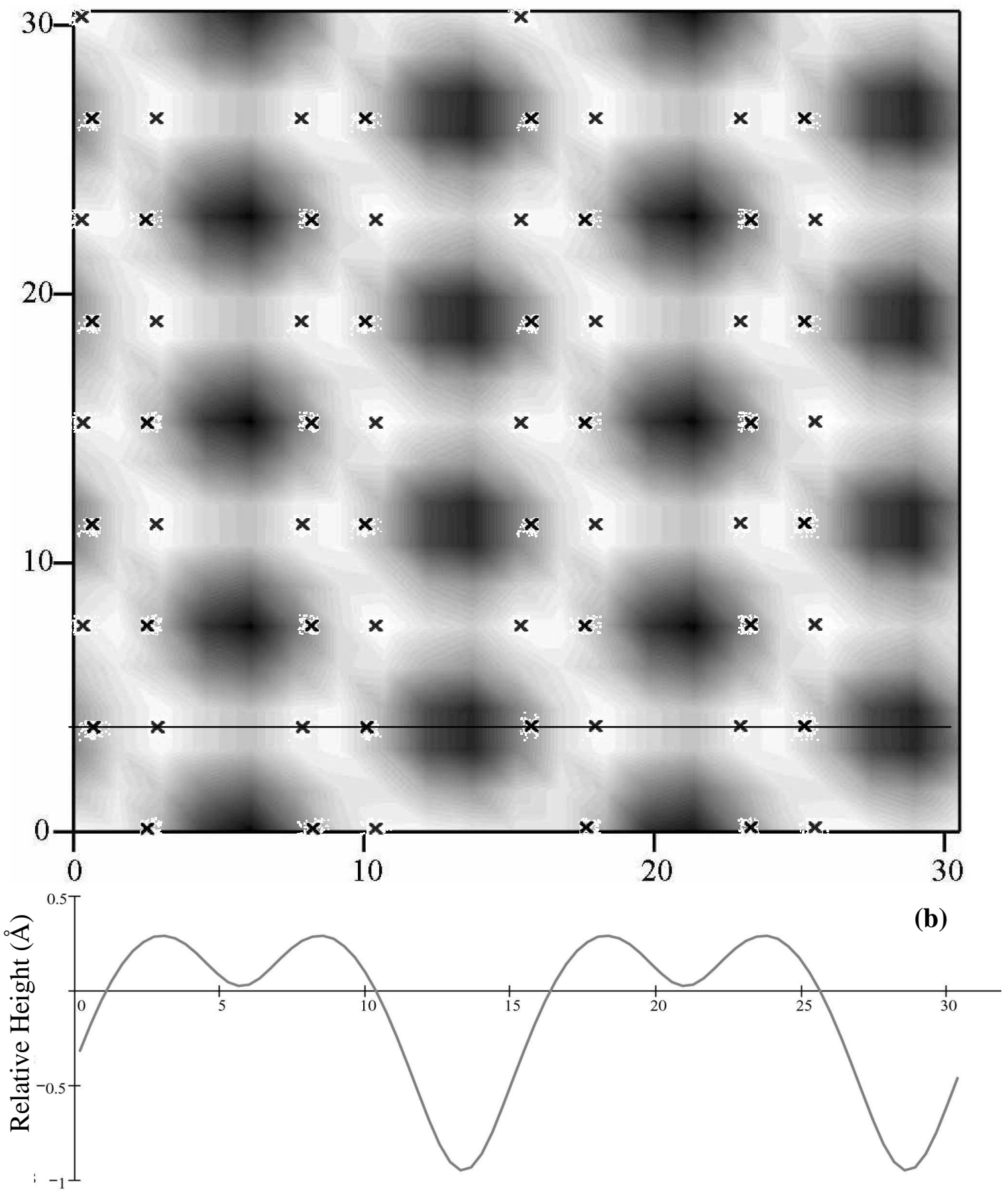


(a)
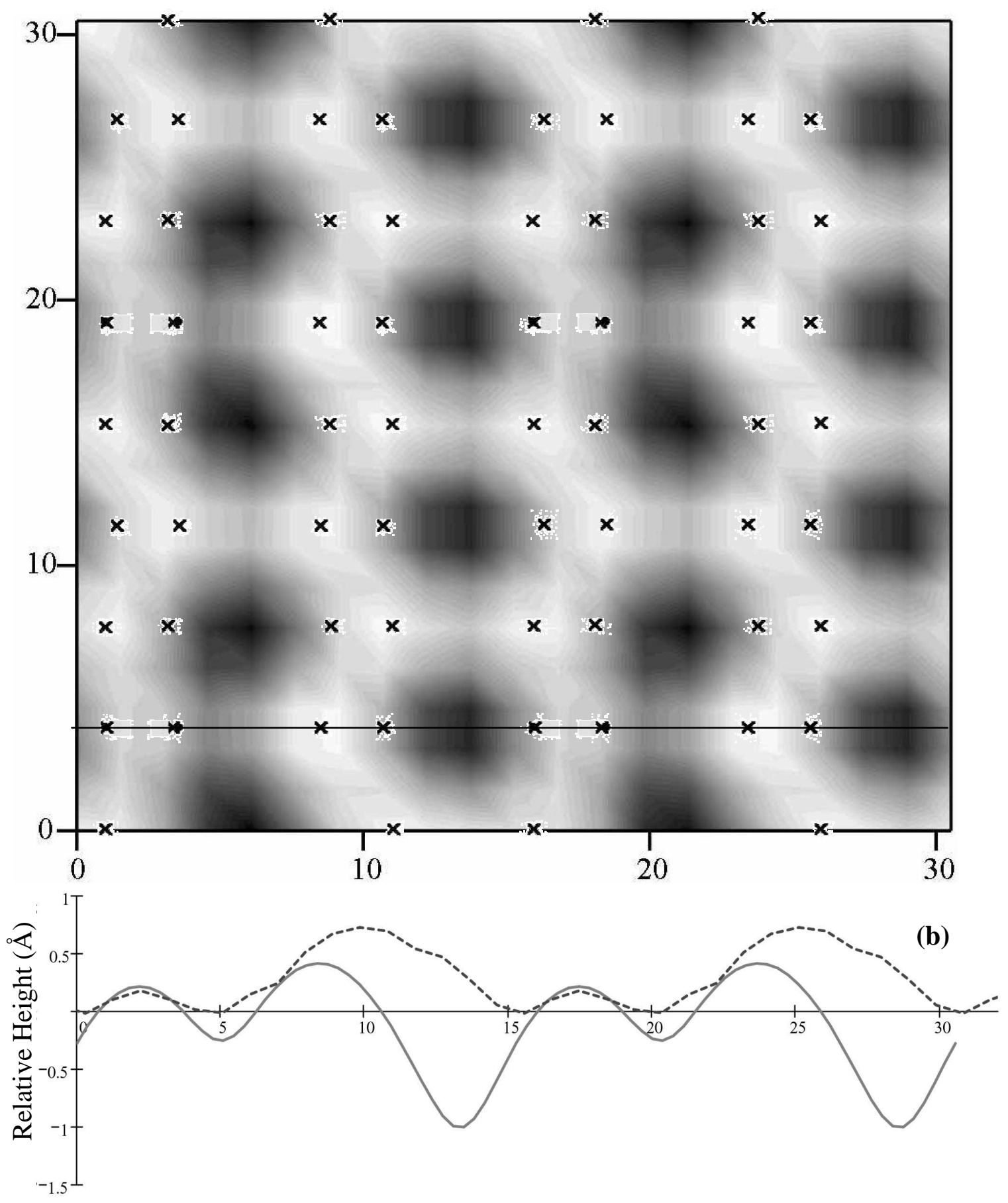
(a)
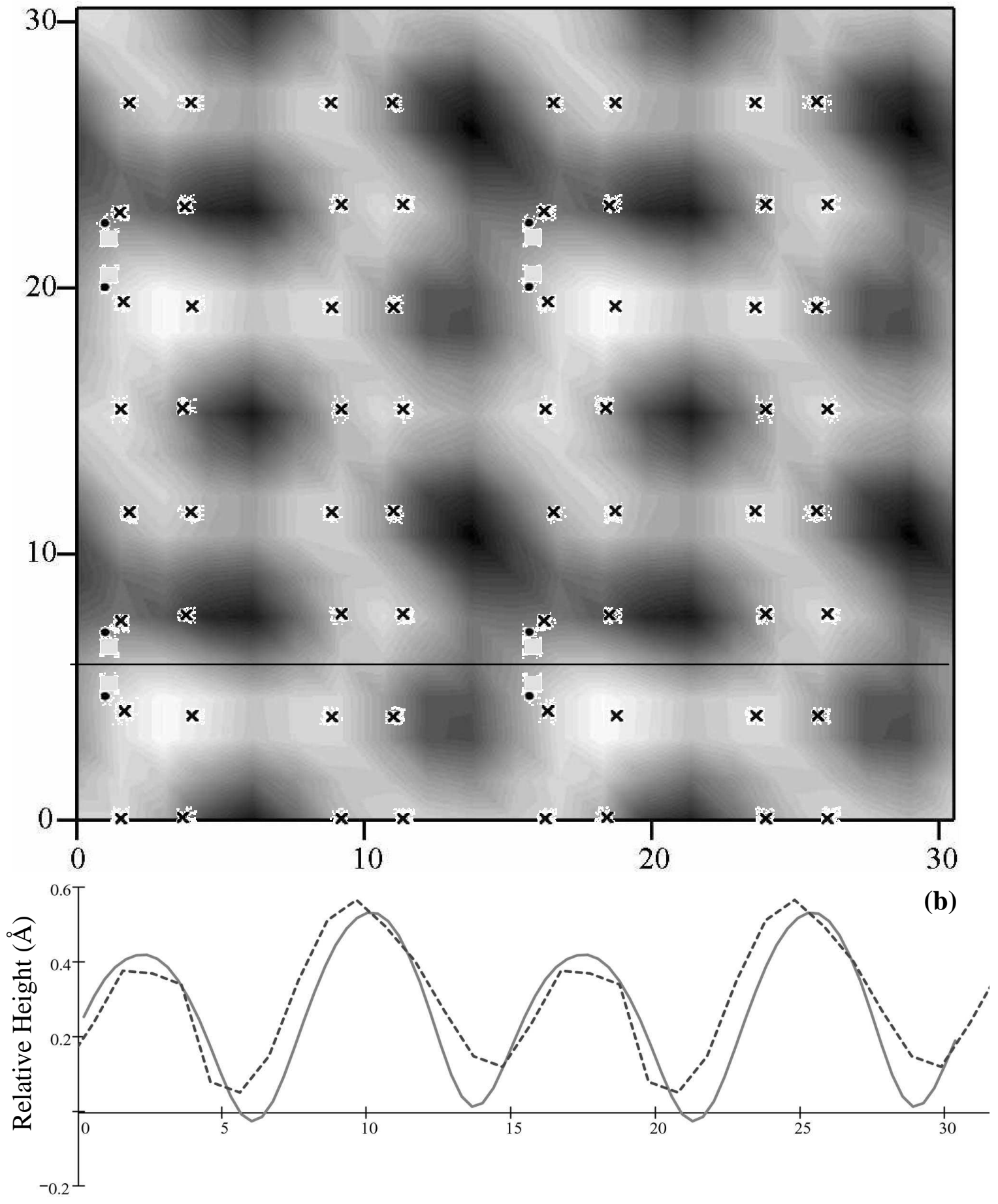
(a)
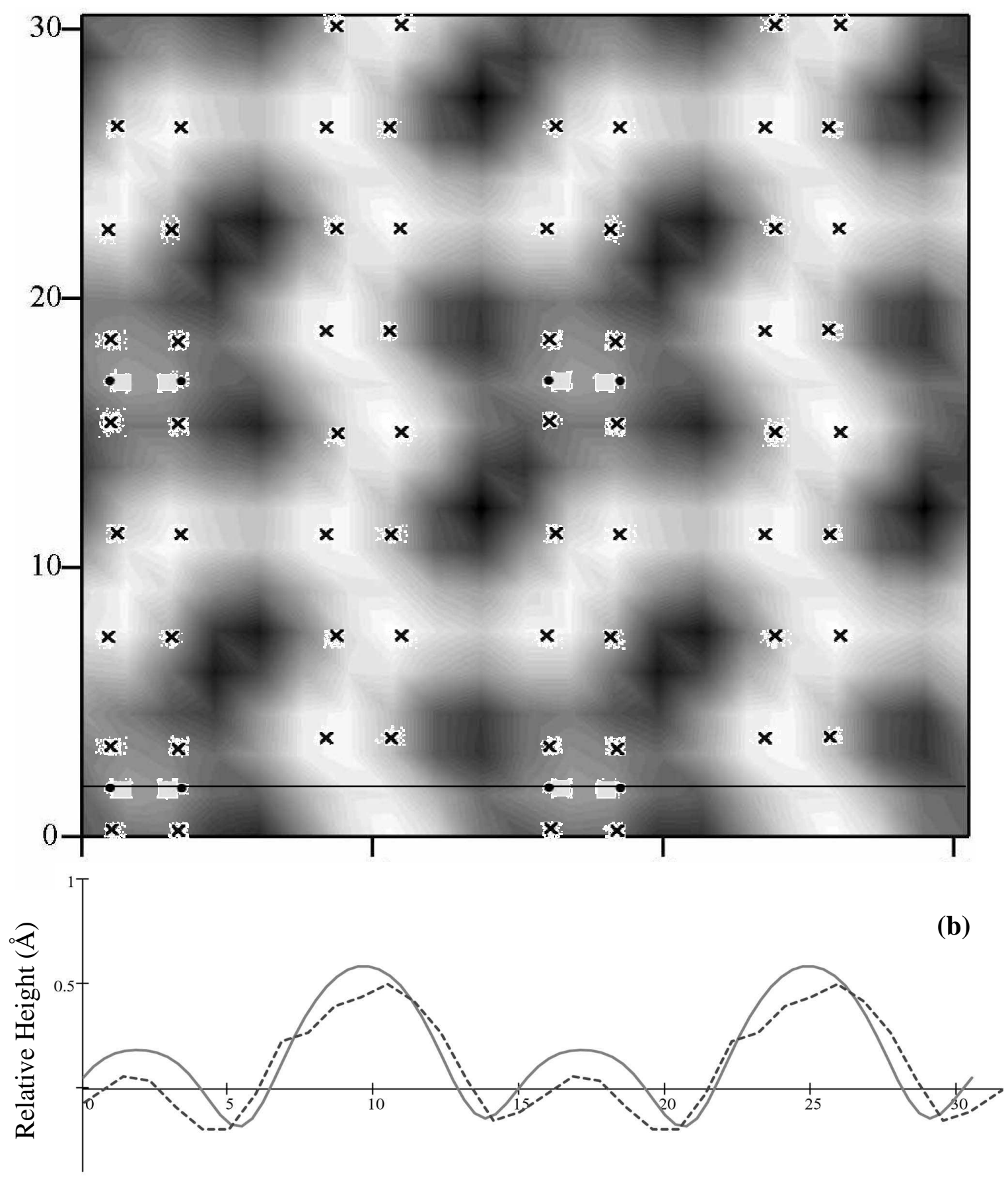
(a)

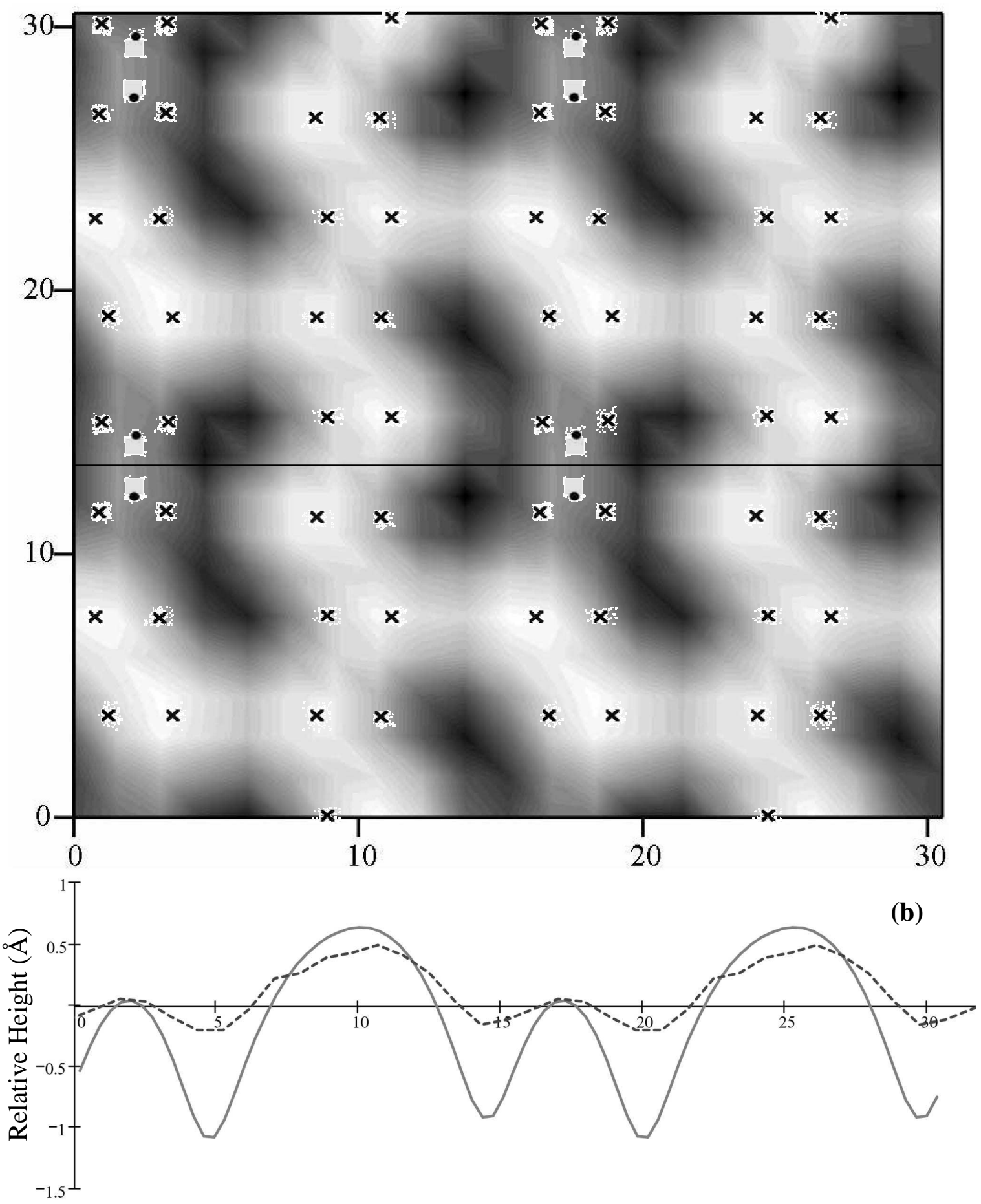


(a)

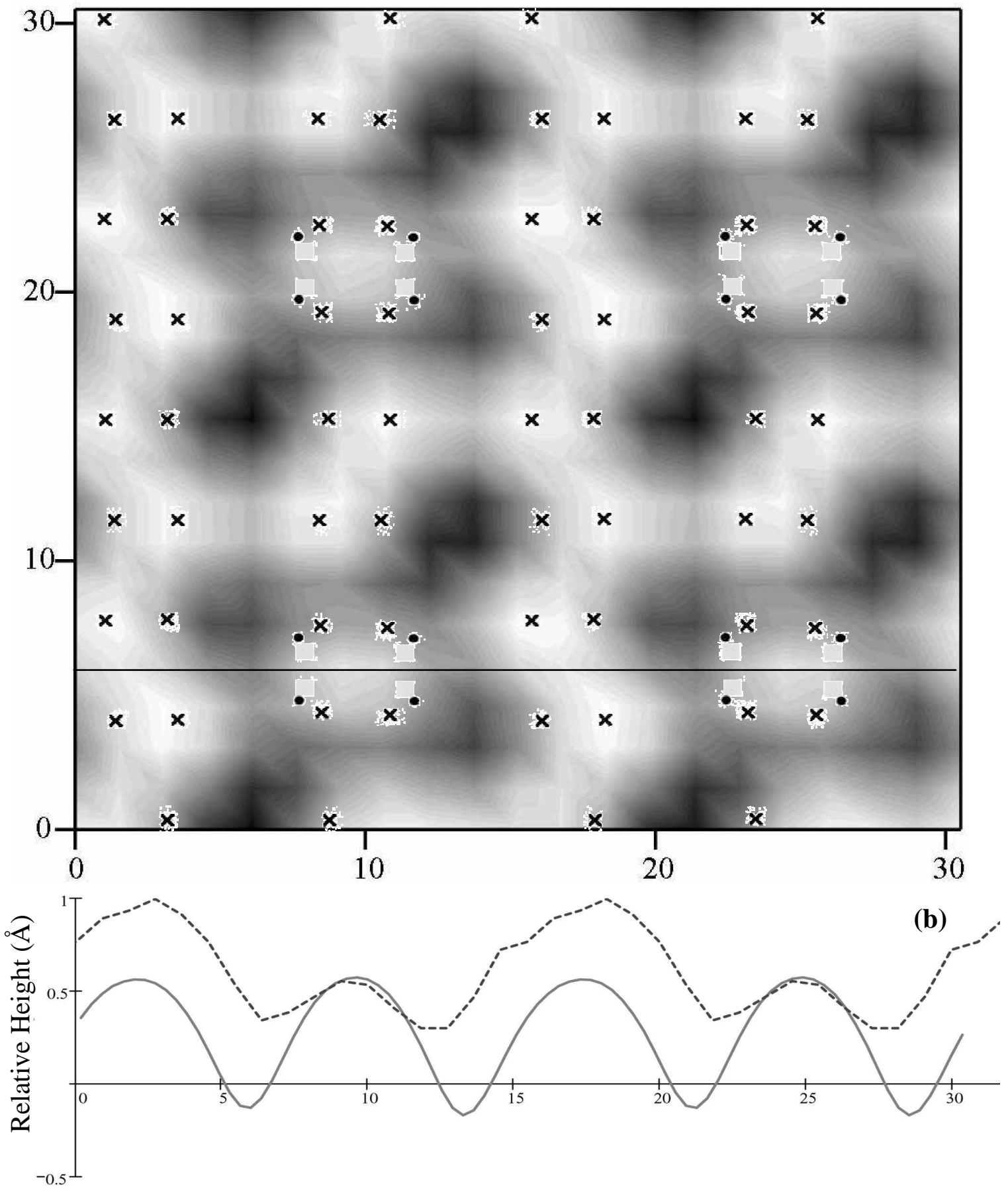

